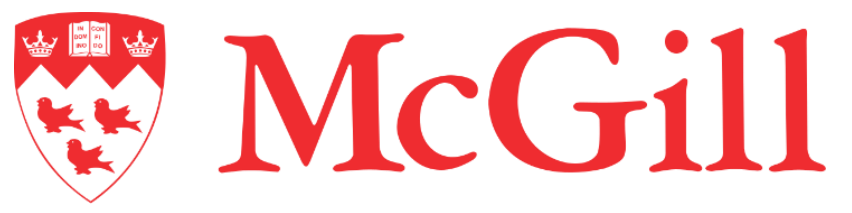

\title{
Patient specific surgical simulation of the ascending thoracic aorta
}

\author{
Justine Garcia \\ Department of Mechanical Engineering \\ McGill University \\ Montreal, Quebec, Canada
}

October, 2019

A thesis submitted to McGill University in partial fulfillment of the requirements for the degree of Doctor of Philosophy in Mechanical Engineering

C) Justine Garcia, 2019 


\section{Table of contents}

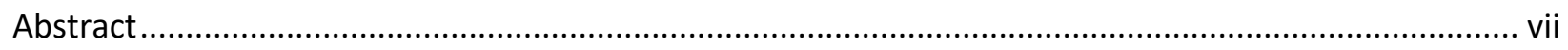

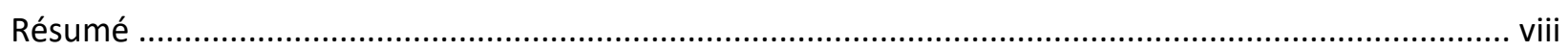

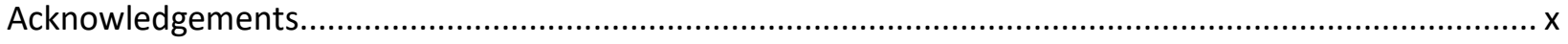

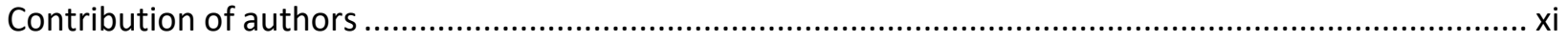

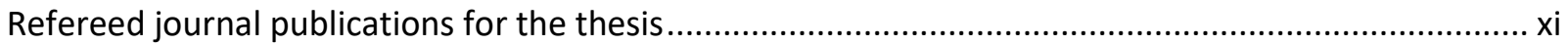

Refereed conference proceedings and abstracts............................................................................ii

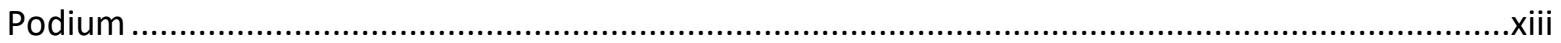

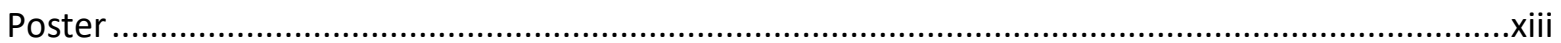

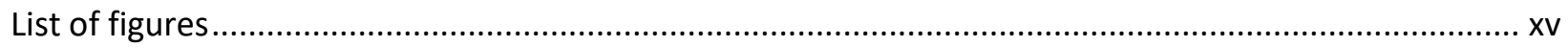

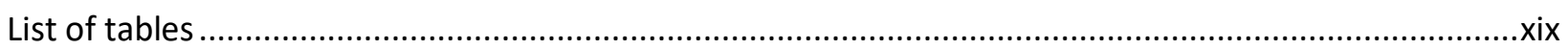

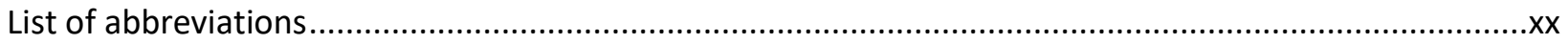

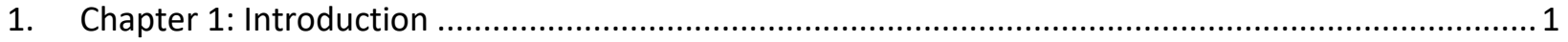

2. Chapter 2: Objectives, research question and overview of the thesis .......................................... 3

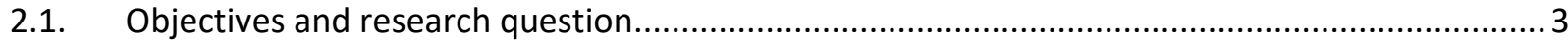

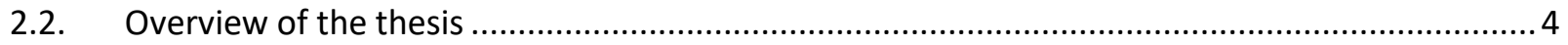

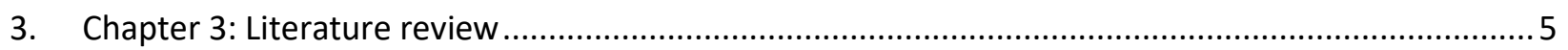

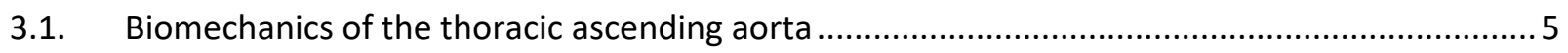

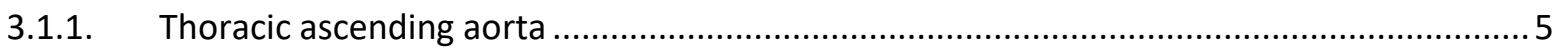

3.1.2. Characteristics of the ascending aortic tissue.............................................................. 5

3.1.3. Alteration of the mechanical properties..................................................................... 6

3.1.4. Characterization of biomechanical parameters defining the aorta................................... 7

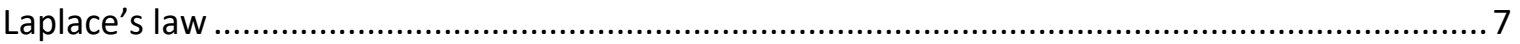

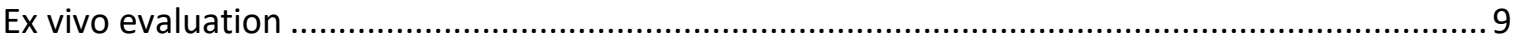

Measurement of the tissue biomechanics............................................................................ 9

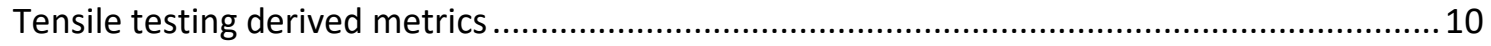

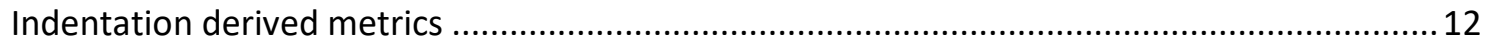

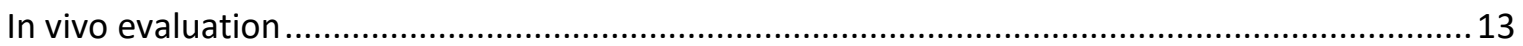

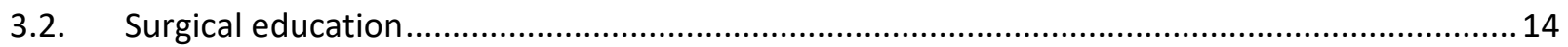

3.2.1. Medical simulation: an increasingly popular educational method................................... 14

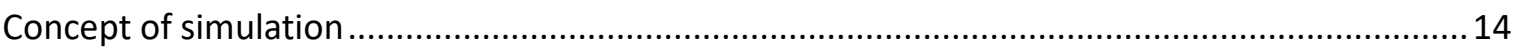

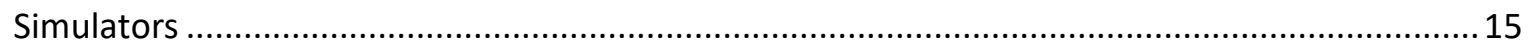

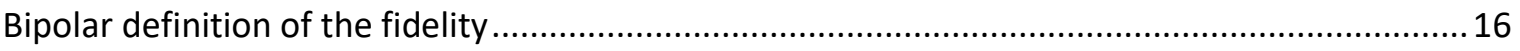




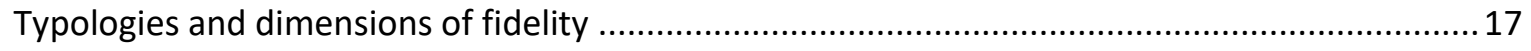

3.2.2. State-of-the-art of vascular phantoms …................................................................... 18

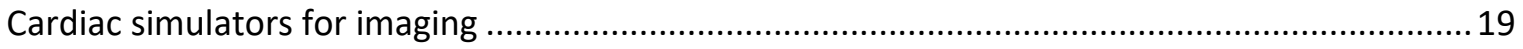

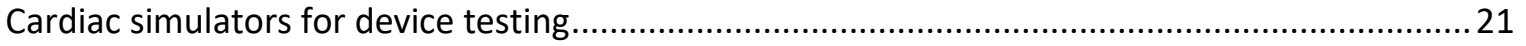

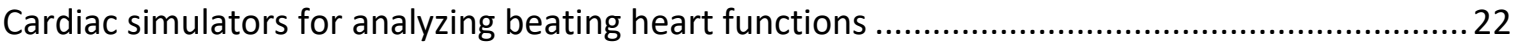

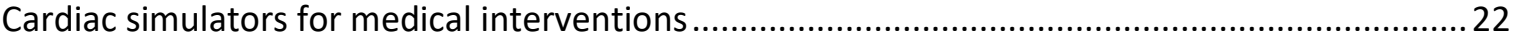

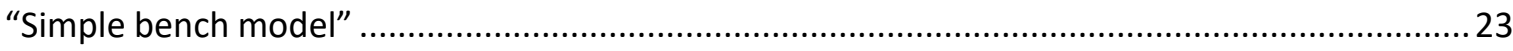

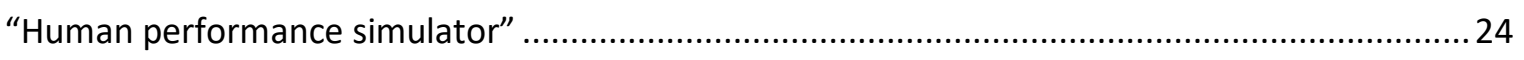

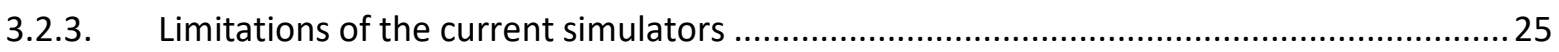

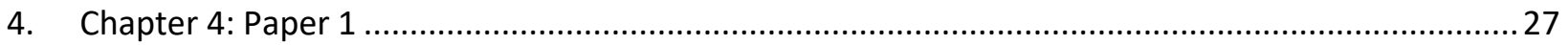

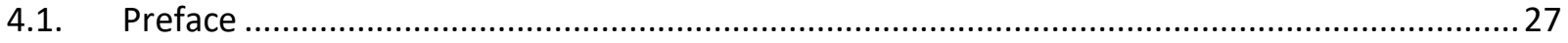

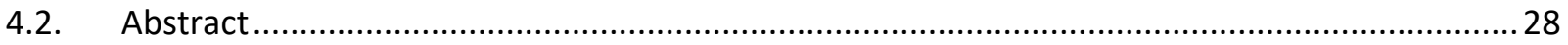

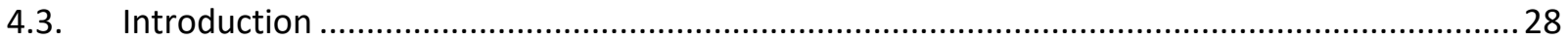

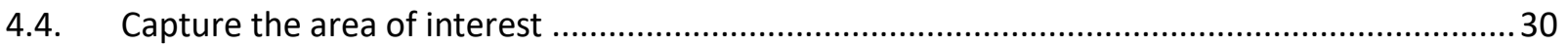

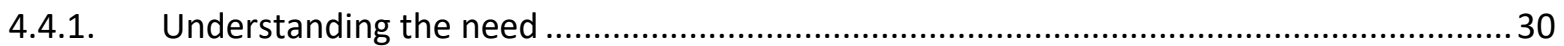

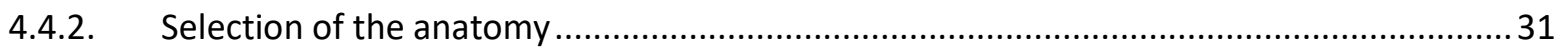

4.5. Creation of 3D geometry from dataset specific to the area of interest ....................................... 34

4.6. Transformation of the 3D object to a file ready for printing .................................................. 36

4.7. Selection of the appropriate 3D printer …...................................................................... 40

4.7.1. Description of the 3D printing methods ....................................................................... 40

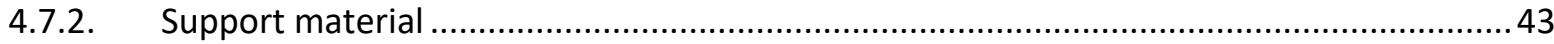

4.7.3. Costs of the printing: 3D printer, material and technical support................................... 44

4.7.4. Building speed, accuracy and quality of the fabrication ...................................................46

4.7.5. Selecting the right printer for teaching...................................................................... 47

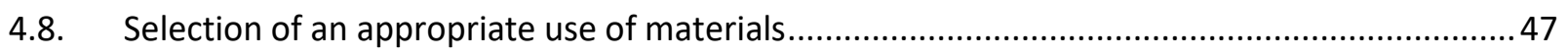

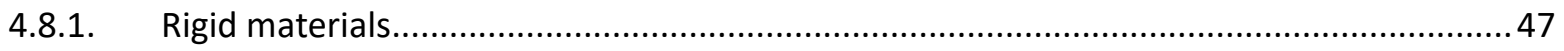

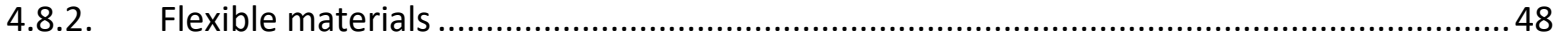

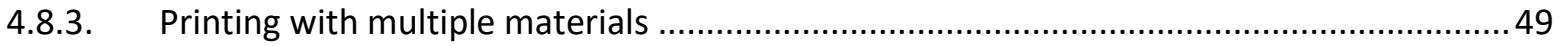

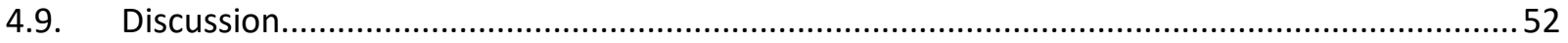

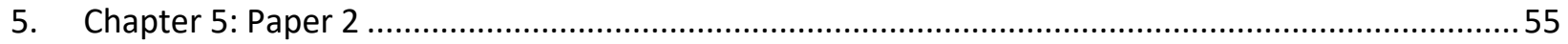

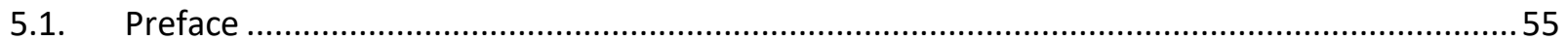

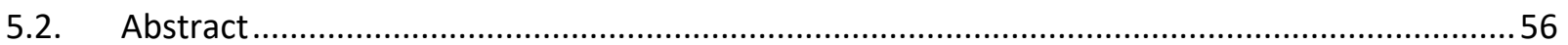

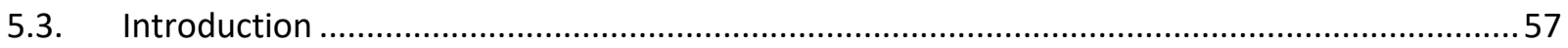




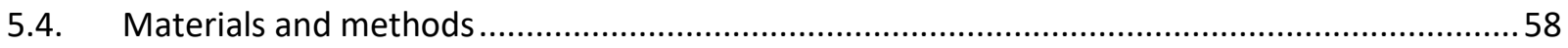

5.4.1. Creation of the heterogeneous synthetic aortic tissue .................................................58

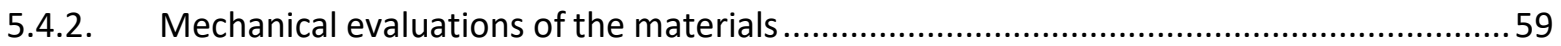

5.4.3. Apparent elastic modulus and energy loss of the material: equi-biaxial tensile testing ....60

5.4.4. Compressive modulus: nano-indentation testing (ASTM E2546) ....................................60

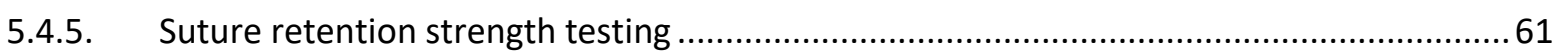

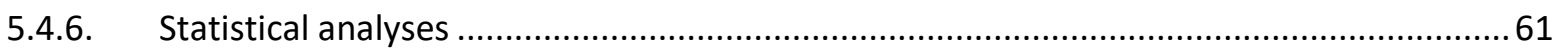

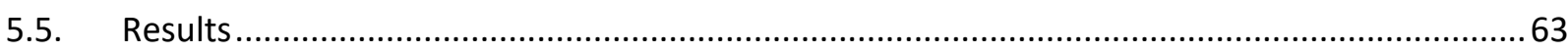

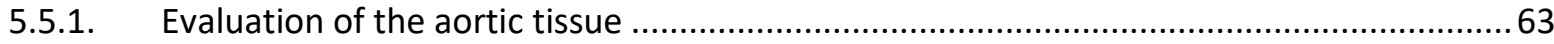

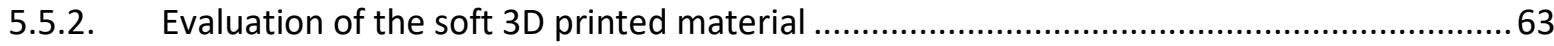

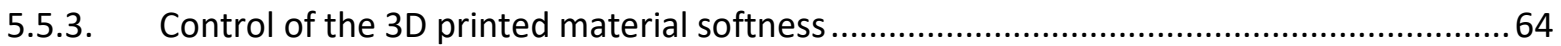

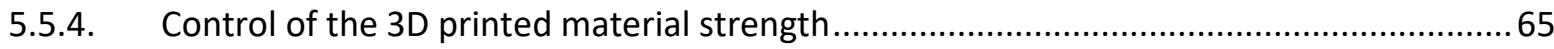

5.5.5. Control of the mechanical directional dependency of the 3D printed material ...............66

5.5.6. Tunable, soft but strong three-material composite ......................................................67

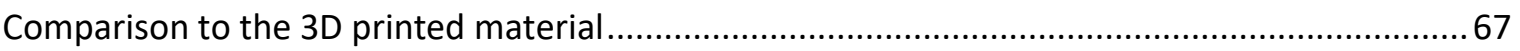

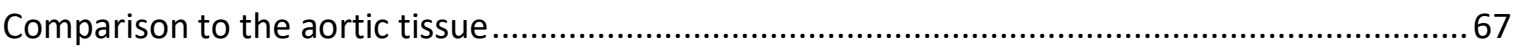

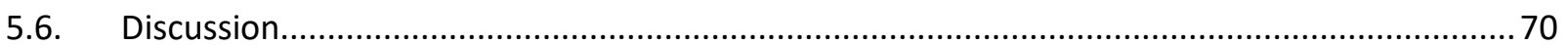

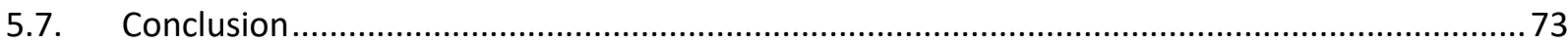

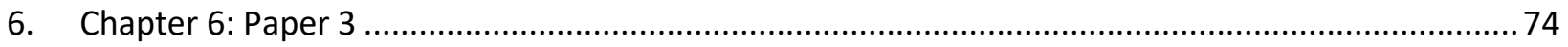

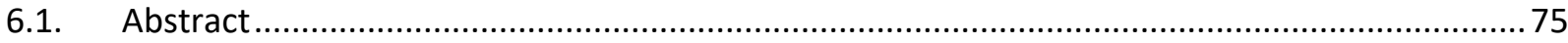

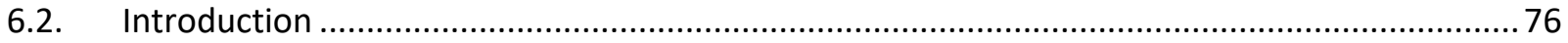

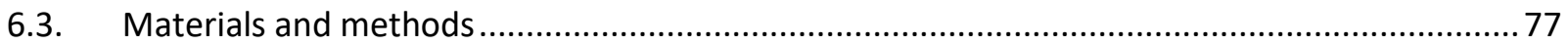

6.3.1. Geometries and materials of the 3D printed aortic simulators ......................................77

6.3.2. Echocardiography and dynamic speckle tracking ........................................................ 78

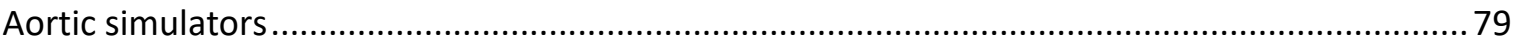

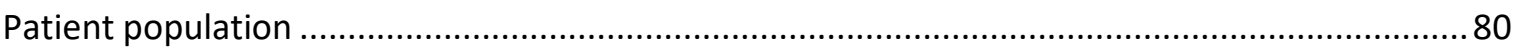

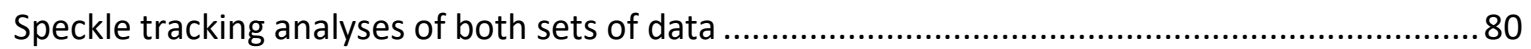

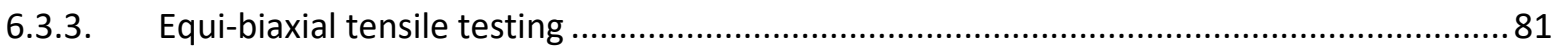

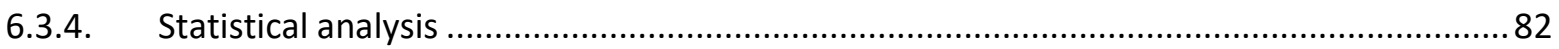

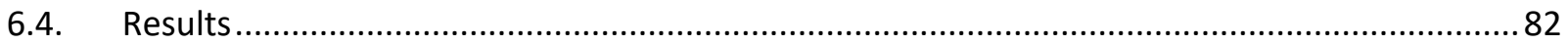

6.4.1. Effects of the geometry and material on the model tensile properties ............................83

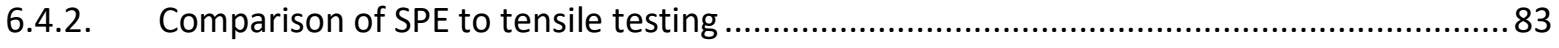

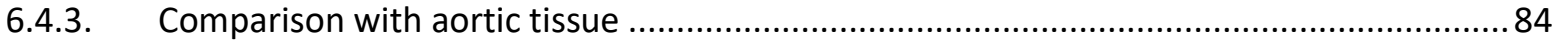




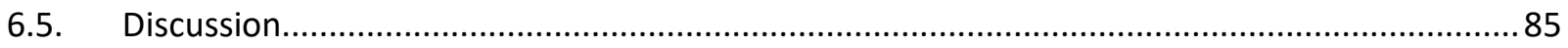

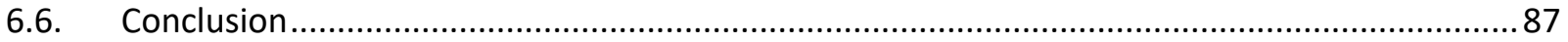

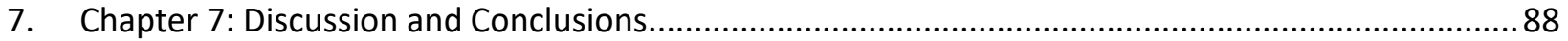

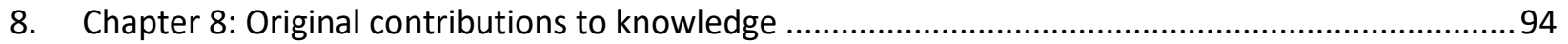

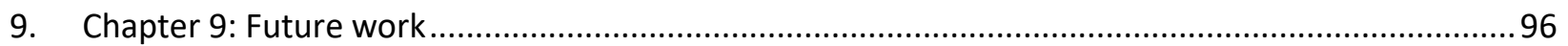

9.1. Aortic model and more detailed 3D printed tissue ..............................................................96

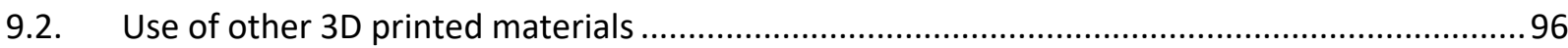

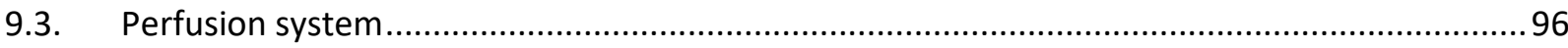

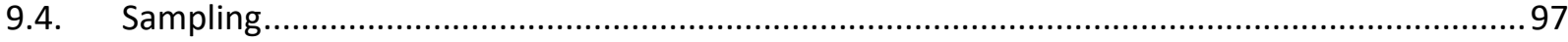

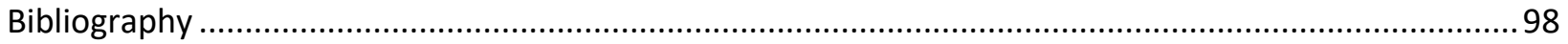

Appendix

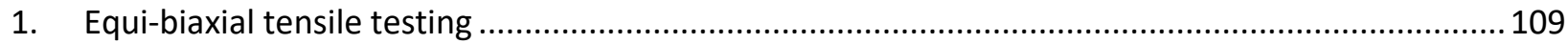

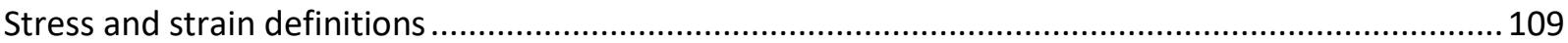

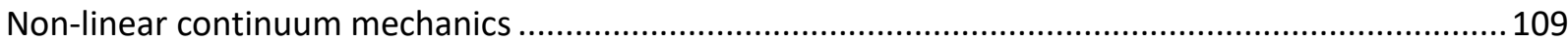

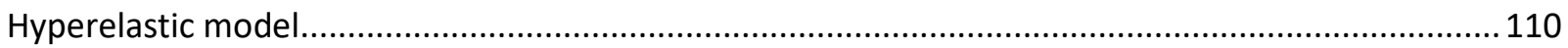

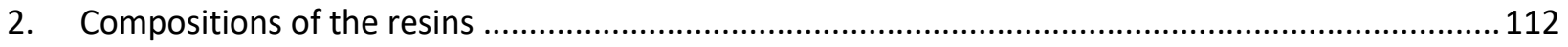

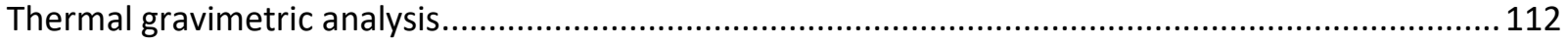

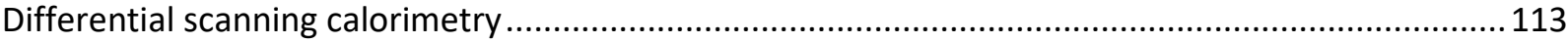

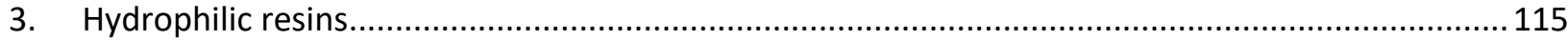

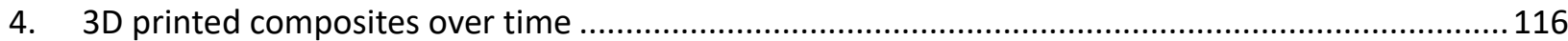

5. Implementation of the composite structure in the aortic geometries for static mechanically realistic

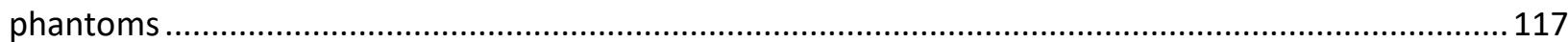

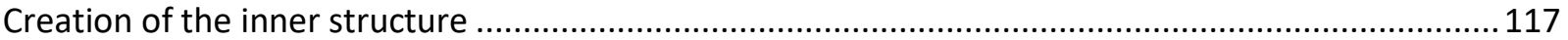

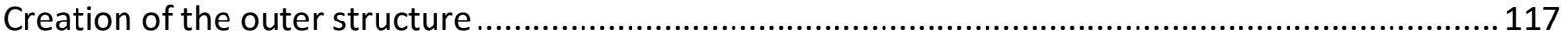

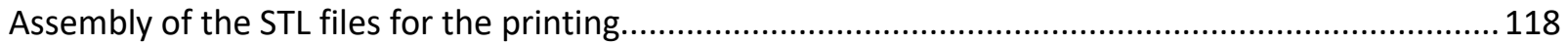

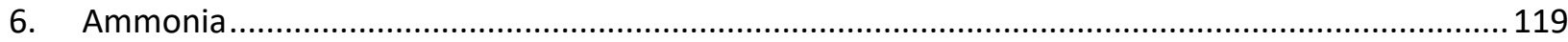

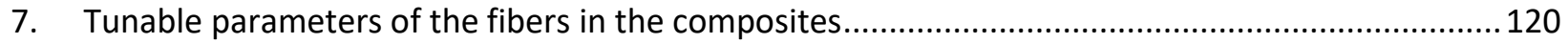




\section{Abstract}

Simulations used for open cardiac surgical procedures usually involve an idealized phantom composed of commercial tissue substitutes or expensive single and cadaveric models. However, current phantoms lack patient specific pathologies and are often crude tubular models. Their mechanical properties are also very unrealistic and none of the present models are designed to be perfused in a physiologic manner. There is, therefore, a need for realistic physical training simulators to help surgeons to practice techniques, thus improve the procedures and patient safety.

3D printing is a very promising and burgeoning field in medicine. It is an additive manufacturing process which is well suited to improving surgical training phantoms especially if one can create patient specific models which replicate the actual normal and pathological tissue characteristics and patient-specific geometries. An important aspect of 3D printed models used in surgical training is their limited material properties. To date, the simulators available lack the material properties needed to mimic the complex tissue properties of ascending aorta and the manipulations required during aortic surgery. For these reasons, the novel aspect of this thesis is to focus on the creation of synthetic composite materials that would be implemented in patient-specific geometries and would mimic the biomechanics of in vivo and ex vivo aortic tissue (normal and pathological).

The research reported in this thesis aimed at establishing methods to create 3D printed aortic models with anatomical, tissue and physiological fidelities. Accurate patient-specific geometries were obtained by gated CT-Scan while the design of 3D printed and tunable composites was guided by the composition of the aortic tissue. Mechanical evaluations of material samples were performed by tensile testing, nanoindentation and suture retention strength tests. It was found that characteristics of the printed materials could be controlled, such as the hardness, strength and mechanical directional dependency.

To demonstrate in vivo fidelity, a two-material composite was implemented into an aortic simulator and evaluated by echocardiographic speckle track analyses. The simulated tissue showed a good echogenicity and matched in vivo properties of patient aortas.

This work demonstrates how a new generation of aortic simulators with patient-specific geometries can be created leveraging 3D printed tunable materials. It shows that such simulators can replicate in vivo as well as ex vivo aortic properties for an enhanced surgical simulation training. 


\section{Résumé}

Les outils de simulations utilisés pour de la chirurgie cardiaque sont généralement des simulateurs aux géométries simplistes, ou bien des cadavres dont la quantité diminue au fil des ans et coûtent très chers. Cependant ces modèles synthétiques manquent de représentativité car ils n'ont que très rarement des formes pathologiques. On retrouve notamment des tubes très primaires pour simuler aussi bien des vaisseaux que des aortes. Les propriétés mécaniques de ces modèles ne simulent également pas bien le comportement des véritables tissus et aucun des simulateurs aujourd'hui ne peuvent subir une perfusion aortique. Il y a donc un véritable besoin de créer de nouveaux outils de formation pour aider les cardiochirurgiens à pratiquer leurs opérations afin d'en améliorer les gestes, les techniques et de diminuer les risques pour le patient.

L'impression 3D est un domaine en pleine expansion, notamment en médecine. Il s'agit d'une méthode de fabrication par ajout de matière qui permette une amélioration des actuels simulateurs chirurgicaux. Cependant, la principale limite de cette technologie se trouve au niveau de ses matériaux qui ne peuvent actuellement pas imiter la complexité des tissus pour de la simulation chirurgicale. Cette thèse repose donc sur la création d'un matériau composite synthétique qui puisse être implémenté pour simuler du tissu aortique (in vivo et ex vivo) dans des géométries obtenues à partir d'images de patients.

Le projet de recherche de cette présente thèse a donc pour objectif de créer des modèles aortiques imprimés en 3D ayant des fidélités d'ordre anatomique, mécanique (représentativité du tissu) et physiologique.

Des géométries d'aortes de patients ont été obtenues à partir d'images CT-scan alors que le développement d'un matériau composite s'est fait en se basant sur la composition du véritable tissu. Les tests mécaniques effectués sur des échantillons de matériaux étaient en traction, compression et résistance à la suture. Nous avons trouvé que nous en avions pu contrôler la résistance en traction, compression et l'isotropie/ anisotropie.

Le tissu synthétique a été ensuite implémenté dans une forme aortique pour une analyse par une analyse échocardiographique de suivi des marqueurs acoustiques. Le matériau a également montré une bonne capacité à être échogène et des similitudes de comportement mécanique avec de véritables aortes.

Ces analyses ont donc démontré que les propriétés mécaniques de nos modèles permettent une bonne simulation d'aortes en conditions in vivo et ex vivo. Nous avons donc été en mesure de développer une 
nouvelle génération de modèles aortiques faits d'un matériau composite dont les propriétés peuvent être contrôler afin d'améliorer la formation des cardio-chirurgiens. 


\section{Acknowledgements}

I would like first and foremost express my sincere gratitude to my advisors: Prof Rosaire Mongrain, Prof Richard Leask and Dr Kevin Lachapelle for your guidance, advice, support and patience. I know that it might have been hard for you to understand me sometimes - as a native French speaker - but you never stopped encouraging and helping me over the years. It was an honor to achieve this project under your supervision. Thank you for believing in me.

Many thanks to the members of the lab who have been very supportive along this journey. I would like to give my sincere appreciation to ZhiLin Yang, Mansour AlOmran, Ali Alakhtar, Cornelius Hart and Alexander Emmott for their collaboration in this project.

Mais par-dessus tout, je souhaite remercier mes proches sans qui je ne serais là aujourd'hui. Ma famille qui m'a toujours soutenu dans mes études et mon envie d'aller vivre à l'autre bout du monde.

Finally, I would also like to acknowledge the McGill Faculty of Engineering who funded the project with the McGill Engineering Doctorate Award (MEDA). 


\section{Contribution of authors}

\section{Refereed journal publications for the thesis}

This thesis is presented in a manuscript-based format containing two published manuscripts and one that still ongoing. I started my PhD project in collaboration with ZhiLin Yang (Master's student) for a year and continued with Mansour AlOmran, Ali Alakhtar (residents in cardiac surgery) and Cornelius Hart for the final testing.

As a second author, I published with Alexander Emmott the following paper where I helped collect the data, write and edit the manuscript:

Emmott, A., J. Garcia, J. Chung, K. Lachapelle, I. El-Hamamsy, R. Mongrain, R. Cartier, and R. L. Leask. (2016). Biomechanics of the ascending thoracic aorta: A clinical perspective on engineering data., Canadian journal of cardiology, 32: 35-47.

Parts of this manuscript appear in the introduction of this thesis.

The models created in this thesis were envisioned and designed in consultation with my supervisors (Prof Rosaire Mongrain, Prof Richard L. Leask and Dr Lachapelle). The modeling, testing and additive manufacturing were performed by me.

\section{Manuscript 1 (Chapter 4)}

Garcia, J., Yang, Z., Mongrain, R., Leask, R. L., \& Lachapelle, K. (2017). 3D printing materials and their use in medical education: a review of current technology and trends for the future. BMJ Simulation and Technology Enhanced Learning. doi:10.1136/bmjstel-2017-000234

Justine Garcia: Literature review, Data collection, 3D printing, Software, Materials, Manuscript writing ZhiLin Yang: Literature review, 3D printing, Software Rosaire Mongrain: Supervision, Materials, Software Richard L. Leask: Supervision, Study Design, Materials, Manuscript review Kevin Lachapelle: Supervision, Study Design, Surgical education, 3D printing, Manuscript writing and review 


\section{Manuscript 2 (Chapter 5)}

Garcia, J., M. AlOmran, A. Emmott, R. Mongrain, K. Lachapelle, and R. L. Leask. (2018). Tunable 3D printed multi-material composites to enhance tissue fidelity for surgical simulation., Journal of Surgical Simulation, 5: 87-98.

Justine Garcia: Computer-aided design, 3D printing, Design of experimental protocols, Mechanical evaluations, Statistical analyses, Codes with Matlab

Mansour AlOmran: Study design, Surgical education

Rosaire Mongrain: Supervision, Materials, Software

Kevin Lachapelle: Supervision, Study Design, Surgical education, 3D printing, Manuscript review

Richard L. Leask: Supervision, Study Design, Materials, Manuscript review

\section{Manuscript 3 (Chapter 6)}

Garcia, J., A. Alakhtar, M. AlOmran, C. Hart, A. Emmott, Mongrain R., R. L. Leask, and Lachapelle K. (2019) 3D Printed dynamic and ultrasound-compatible ascending aortic simulators for surgical simulation.

Justine Garcia: Computer-aided design, 3D printing, Design of experimental protocols, Mechanical evaluations, Statistical analyses, Codes with Matlab

Ali Alakhtar: Surgical education, Medical expertise

Mansour AlOmran: Study design, Surgical education, Medical expertise

Cornelius Hart: Computer-aided design, 3D printing, Mechanical evaluations

Alexander Emmott: Mechanical evaluations

Rosaire Mongrain: Supervision, Materials, Software

Kevin Lachapelle: Supervision, Study Design, Surgical education, 3D printing, Manuscript review

Richard L. Leask: Supervision, Study Design, Materials, Manuscript review 


\section{Refereed conference proceedings and abstracts}

Podium

- Garcia, J., Cornelius Hart, ZL. Yang, K. Lachapelle, R. Mongrain \& Leask R. L. (September 28, 2018). Simulated aortic tissue using 3D printed multi-material composites for surgical training. Paper presented at the Canadian Aortic and Vascular Engineering Symposium. Montreal, Canada. (presented by Cornelius Hart)

- $\quad$ Garcia, J., ZL. Yang, K. Lachapelle, R. Mongrain \& Leask R. L. (August 14-17, 2018). Simulated aortic tissue using 3D printed multi-material composites for surgical training. Paper presented at the Canadian Society for Biomechanics. Halifax, Canada.

- $\quad$ Garcia, J., Yang, Z., Lachapelle, K., Leask, R. L., \& Mongrain, R. (May 5, 2016). 3-D printing in cardiac surgery. Paper presented at the Stikeman Visiting Professor for Cardiovascular and Thoracic Surgery, Montreal, Canada.

- $\quad$ Garcia, J., Yang, Z., Lachapelle, K., Mongrain, R., \& Leask, R. L. (October 24-27, 2015). Physical patient specific simulation for ascending aortic aneurysms surgery pre-procedural training. Paper presented at the Canadian Cardiovascular Congress Toronto, Canada.

- $\quad$ Garcia, J., Yang, Z., Lachapelle, K., Mongrain, R., \& Leask, R. L. (June 17-20, 2015). Physical patient specific simulation for ascending aortic aneurysms surgery pre-procedural training. Paper presented at the Summer Biomechanics, Bioengineering \& Biotransport Conference, Snowbird Resort Utah, USA.

Poster

- $\quad$ Garcia, J., Yang, Z., Lachapelle, K., Mongrain, R., \& Leask, R. L. (November 1-2, 2017). Simulated aortic tissue using 3D printed multi-material composites Paper presented at the Simulation summit, Montreal, Canada. (Simulation Summit Research Award for the best presentation)

- Garcia, J., Yang, Z., Lachapelle, K., Mongrain, R., \& Leask, R. L. (November 18, 2016). Physical patient specific simulation for ascending aortic aneurysms surgery pre-procedural training. Paper presented at the Quebec Society of Vascular Science, Montreal, Canada.

- $\quad$ Garcia, J., Yang, Z., Lachapelle, K., Mongrain, R., \& Leask, R. L. (October 22-24, 2016). Patient specific surgical simulation of the thoracic aorta. Paper presented at the Canadian Cardiovascular Congress, Montreal, Canada. 
- Garcia, J., Yang, Z., Lachapelle, K., Mongrain, R., \& Leask, R. L. (June 5, 2016). Patient specific simulation for aortic surgery pre-procedural training. Paper presented at the Research Day of the Montreal Heart Institute, Montreal, Canada. (Martial Bourassa award for the best presentation)

- Garcia, J., Yang, Z., Lachapelle, K., Mongrain, R., \& Leask, R. L. (March 3, 2016). Patient specific simulation for aortic surgery pre-procedural training. Paper presented at the Mechanical Engineering Research Day, Montreal, Canada.

- Garcia, J., Yang, Z., Lachapelle, K., Mongrain, R., \& Leask, R. L. (June 4, 2015). Patient specific simulation for aortic surgery pre-procedural training. Paper presented the Research Day of the Montreal Heart Institute, Montreal, Canada.

- $\quad$ Garcia, J., Yang, Z., Lachapelle, K., Mongrain, R., \& Leask, R. L. (March 13-14, 2015). Patient specific simulation for aortic surgery pre-procedural training. Paper presented at the Annual ACS AEI Consortium Meeting, Chicago, USA. 


\section{List of figures}

Figure 1. a) Typical geometries of a human heart and b) an aorta with the blood flow directions illustrated

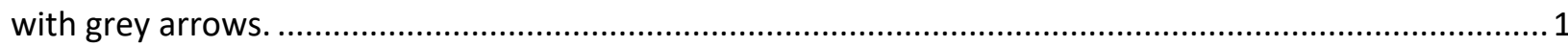
Figure 2. Histopathology of the ascending aorta using Movat pentachrome staining, a) in a 52-year-old man with a nondilated ascending aorta and b) a 51-year-old man with a 5.8-cm dilated ascending thoracic aortic aneurysm. Black indicates elastin; red/purple, smooth muscle cells; blue, mucopolysaccharides; and yellow, collagens. Reprinted with permission (Emmott et al., 2016)...................................................6 Figure 3. Illustration of Laplace's law: wall tensions in the vessel (simplified by a cylinder with a radius $r$ larger than a wall thickness $\tau$ ) when pressure $P$ is exerted by the aortic blood flow. $P$ is assumed evenly distributed over the wall. $\sigma_{\theta}$ is the circumferential stress. Reprinted with permission (Emmott et al., 2016).

Figure 4. Typical shape of stress-strain curve for linear elastic (green line) and visco-elastic response of biological tissue (black line). (A) Elastic modulus of a linear elastic material (E), (B Elastic modulus of a nonlinear material $\left(E_{m}\right)$ and $(C)$ Energy loss.

Figure 5. Typical loading and unloading curves of the load and penetration-depth with visco-elastic materials in indentation. $P_{\max }$ is the maximum load, $\mathrm{h}_{\max }$ the maximum depth and $\mathrm{Si}$ the stiffness of the material calculated with the slope of the beginning of the unloading curve...... 13 Figure 6. a) An ultrasound torso for medical image acquisition training (Blue Phantom, 2013) and b) an ultrasound torso with removable organs for transthoracic echocardiography ultrasound training (Computerized Imaging Reference Systems Inc., 2014). .................................................................20 Figure 7. a) An anatomically correct heart silicone model for medical imaging technologies (Boltz et al., 2010), b) a non-anatomically correct left ventricle model made of silicone gum for cardiac positron emission tomography and single photon emission computed tomography (Presotto et al., 2012), c) a ventricle made of balloons for positron emission tomography (Sipila et al., 2007) and d) a nonanatomically correct heart model made of polyvinyl alcohol cryogel for ultrasound and cardiac magnetic resonance (Tavakoli et al., 2012).

Figure 8. a) A hypoplastic aorta and a right ventricle printed in Tangoplus Fullcure ${ }^{\circledR}$ (Biglino et al., 2013) and $\mathrm{b}$ a) stenotic cross-section of a coronary polyvinyl alcohol cryogel phantom (image and geometry).Reprinted with permission (Pazos et al., 2010). ..................................................................22 Figure 9. Physical assessment trainers with sounds of beating hearts: a) Koken Co.(Koken Co., 2005) b) Harvey ${ }^{\circledR}$ The Cardiopulmonary Patient Simulator with sounds of beating hearts, Laerdal Medical (Laerdal Medical, 2014), c) Cardiology patient simulator "K", Kyoto Kagaku Co. (Kyoto Kagaku Co., 1999-2012). 22 Figure 10. Heart phantom model for basic tasks (The Chamberlain Group, 2014a).

Figure 11. a) Aorta and vessel models for suture training, b) an aortic root model for valve implantation training, c) a transseptal trainer, d) a catheter insertion trainer, e) a femoral closure wet trainer and f) a cardiac array trainer (The Chamberlain Group, 2014b).....................................................................2 24

Figure 12. Mitral valve annulus model for mitral valve replacement training (McLeod et al., 2011)........24 Figure 13. a) Tissue based simulator with porcine heart for complete surgery training (Ramphal et al., 2005) and b) beating and suture-able heart model (The Chamberlain Group, 2014a)............................25

Figure 14. Steps required in the creation of a 3D printed model in health care education. Reprinted with permission (Garcia, Yang, Mongrain, Leask, \& Lachapelle, 2017). 30 Figure 15. Steps required in the creation of a 3D printed model in health care education. Reprinted with permission (Garcia et al., 2017). 
Figure 16. a) An illustration of the planar 2D images of an area of interest captured by most medical imaging techniques, b) a segmentation of the object cross section (black circles) extracted and c) in interpolation required to fill in the missing volume between segments. Reprinted with permission (Garcia et al., 2017). 32

Figure 17. Two distinct objects made of different materials or printed in different colors creating an assembly. Reprinted with permission (Garcia et al., 2017).

Figure 18. a) A point cloud created from two 2D images and an example of b) a point cloud obtained from the inner lumen of an ascending aorta of a patient from the Royal Victoria Hospital (Montreal, Canada) with a $0.625 \mathrm{~mm}$ thickness between the slices. Reprinted with permission (Garcia et al., 2017).............35 Figure 19. a) Artifacts from the point cloud of an ascending aorta of a patient from the Royal Victoria Hospital (Montreal, Canada) and b) an artifact before and after the mesh smoothing. Reprinted with permission (Garcia et al., 2017).

Figure 20. Spheres created to approximate the shape of a patient-specific aortic root, open source Meshlab (MeshLab, Italy). Reprinted with permission (Garcia et al., 2017). .........................................38 Figure 21. a) Most frequent Boolean operations to create objects (union and subtraction of volumes) and b) volume overlapping that should be avoided in any circumstances. Reprinted with permission (Garcia et al., 2017). 39

Figure 22. Mesh errors of a) non-connecting triangle, b) overlapping triangles and with c) an extra body that is not part of the main geometry. Reprinted with permission (Garcia et al., 2017). 39 Figure 23. Multicolor 3D printed liver with a tumor (pink) of a patient from the Royal Victoria Hospital (Montreal, Canada) for surgical planning of a diseased liver. The print contain the portal vein, the hepatic vein as well as the tumor. Reprinted with permission (Garcia et al., 2017). . .40 Figure 24. Aortic model in process for being printed on a FDM 3D printer by successive layers of ABS plastic (0.3mm thickness). Reprinted with permission (Garcia et al., 2017). 41 Figure 25. Rapid prototyping methods with red arrows indicating the directions of motion ( $x, y, z$ axes). Reprinted with permission (Garcia et al., 2017).

Figure 26. Structure with an overhang filled with a lattice structure, support material or non-cured material. Reprinted with permission (Garcia et al., 2017).

Figure 27. Model of the aorta with root aneurysm for teaching purposes. Reprinted with permission (Garcia et al., 2017).

Figure 28. Head model made of one material to practice the drilling in medical simulation (Chan et al., 2015). Reprinted with permission (Garcia et al., 2017).

Figure 29. Head model made of a combination of materials to practice the drilling in medical simulation Waran et al. (2014). Reprinted with permission (Garcia et al., 2017).

Figure 30. Accurate 3D model of an aortic aneurysm captured from CT imaging (Ho \& Sun, 2017).

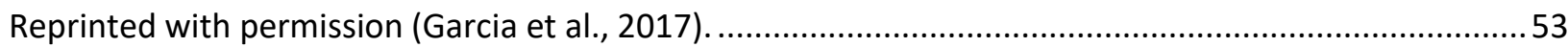
Figure 31. Composition and materials used to control the softness, strength and directional dependency of soft TGPF930 structures, as well as a tunable, soft but strong three-material composite. Reprinted with permission. 59

Figure 32. a) Equi-biaxial tensile test: tensile tissue tester stretching the sample and resulting stress-strain

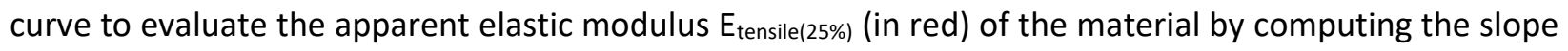
of the loading curve at $25 \%$ strain value. The energy loss was calculated by using the A and B areas, b) nano-indentation test: typical loading and unloading curves of the load and penetration-depth with viscoelastic materials in indentation. $P_{\max }$ is the maximum load, $h_{\max }$ the maximum depth and $\mathrm{Si}$ the stiffness of 
the material calculated with the slope of the beginning of the unloading curve. Nanovea M1 nano-indenter (Nanovea, Irvine, USA) during a test using a spherical tip moving down and touching the surface of the sample, c) suture retention strength: Tensile tester EZ Test (Shimadzu, Columbia, USA) and a suture retention strength test with a 4-0 prolene suture.

Figure 33. a) The apparent elastic modulus $\left(E_{\text {tensile(25\%) }}\right)$, b) energy loss, c) suture retention strength (SRS) and d) compressive modulus ( $E_{\text {comp }}$ ) of pure Tangoplus FullCure 930 (TGPF930) and aortic tissues (normal and aneurysmal). $p$ values of Unpaired t-tests.

Figure 34. a) The suture retention strength (SRS) and (b) compressive modulus ( $E_{\text {comp }}$ ) of pure Tangoplus FullCure 930 (TGPF930) and two-material composite (Tangoplus FullCure 930 (TGPF930) as well as support material (SUP705)). p values of Unpaired t-tests.

Figure 35. a) The apparent elastic modulus $\left(E_{\text {tensile(25\%) }}\right)$, b) energy loss, $c$ ) suture retention strength (SRS) and $d$ ) compressive modulus ( $E_{\text {comp }}$ ) of pure Tangoplus FullCure 930 (TGPF930) and two-material composite (Tangoplus FullCure 930 (TGPF930) with two layers of rigid Vero fibers). $p$ values of Unpaired t-tests. . .66

Figure 36. a) The apparent elastic modulus $\left(E_{\text {tensile(25\%) }}\right)$ and $b$ ) energy loss of the two principal directions of the two-material composite (Tangoplus FullCure (TGPF930) and one layer of large Vero fibers). $p$ values of Paired t-tests.

Figure 37. Comparisons of the three-material composite to the 3D printed composite (a-d) and aortic tissue $(e-h)$ in terms of apparent elastic modulus $\left(E_{\text {tensile(25\%) }}\right)(a, e)$, energy loss $(b, f)$, suture retention strength $(S R S)(c, f)$ and compressive modulus $\left(E_{\text {comp }}\right)(d, h) . p$ values of Unpaired t-tests. 69 Figure 38. Stress and strain loading curves of normal and aneurysmal aortas, as well as three-material composite.

Figure 39 Aortic simulators with the two geometries (tube, aorta or idealized ATAA) and material (TGPF930, Composite). 78 Figure 40. Relative diameters and corresponding pressure obtained for the perfusion system ..............79 Figure 41. a) Piston-pump system for the evaluation of the aortic simulator by SPE and b) Picture of the operator performing the evaluation. .80 Figure 42. a) Points taken along the aortic walls track displacements over time for the septal (sept), anteroseptal (ant sept), anterior (ant), lateral (lat), posterior (post) and interior (int) segments. b) Pressure-circumferential stretch curve to determine the slope, thus the Cardiac cycle pressure modulus (CCPM).

Figure 43. a) Equi-biaxial tensile tester with the generated stress-strain curves obtained from the stretching and b) the Apparent elastic modulus $E_{\text {tensile } 25 \%}$ as well as the Energy loss were calculated with the slope of the loading curve at $25 \%$ strains.

Figure 44. Two-way Anova analyses to compare the effects of geometries and materials to the simulators with the a) Apparent elastic modulus ( $E_{\text {tensile } 25 \%}$ ) and b) Energy loss. .................................................83

Figure 45. Comparison by linear regression of CCPM (Cycle Pressure Modulus) to the a) Apparent elastic modulus ( $E_{\text {tensile } 25 \%}$ ) and b) Energy loss. .84

Figure 46.One-way Anova and Tukey's multiple comparison tests to compare the 3D printed models to patient aortas. .85

Figure 47. Deformation gradient $\mathrm{F}$ from the reference (blue) to the deformed position (green)...........110 Figure 48. Weight-Temperature (green) and Derivative weight-Temperature (blue) curves for TGA analyses of a) Verowhite, b) Tangoplus FullCure 930, the c) Support material, as well as a graphic showing all Weight-Temperature curves in d). 
Figure 49. Heat flow-Temperature curves for DSC analyses of a) Verowhite, b) Tangoplus FullCure 930 and c) Support material. The first heat cycle is in blue, the cool cycle is in red and the second heat cycle in green.

Figure 50. Variations over time of the apparent elastic modulus ( $E_{\text {tensile (25\%) }}(\mathrm{MPa})$ ) and energy loss (\%) for the three-material composite kept in air and nitrogen.

Figure 51. Creation of an intermediate surface over the STL geometry for the projection of one layer of fibers.

Figure 53. a) Fibers embedded in the composite and the b) Tunable parameters (Diameter, Amplitude and Length) of the fibers. 


\section{List of tables}

Table 1 Common cardiac diseases and treatments.

Table 2. Main characteristics of the rapid prototyping methods: Stereolithograpy (SLA), PolyJet (PJ), Fused deposition modeling (FDM), Selective laser sintering (SLS), Binder jetting (BJ). Reprinted with permission (Garcia et al., 2017). .45

Table 3. 3D printing technologies and materials involved in the fabrication of models for surgical training, their simulated body parts, purposes, requirements and the machines used. Reprinted with permission (Garcia et al., 2017). 50

Table 4. Mechanical tests and outcomes obtained from the study. ....................................................60

Table 5. Test applied and outcomes for each tested material. . .70

Table 6. Weights of the samples before and after a 3.5 hours water bath. 115

Table 7. Weights of the samples before and after 3.5 hours. 


\section{List of abbreviations}

$\begin{array}{ll}\text { ATA } & \text { Ascending thoracic aorta } \\ \text { ATAA } & \text { Ascending thoracic aortic aneurysm } \\ \text { BAV } & \text { Bicuspid aortic valve } \\ \text { BJ } & \text { Binder jetting } \\ \text { CT } & \text { Computed tomography } \\ \text { DICOM } & \text { Digital imaging and communication in medicine } \\ \text { DSC } & \text { Differential scanning calorimetry } \\ \text { FDM } & \text { Fused deposition modeling } \\ \text { HF } & \text { High fidelity } \\ \text { LF } & \text { Low fidelity } \\ \text { MRI } & \text { Magnetic resonance imaging } \\ \text { PJ } & \text { Polyjet } \\ \text { PVA } & \text { Polyvinyl alcohol cryogel } \\ \text { SLA } & \text { Stereolithography } \\ \text { SLS } & \text { Selective laser sintering } \\ \text { SPE } & \text { Speckle tracking echochardiography } \\ \text { STL } & \text { Stereolithography } \\ \text { TAV } & \text { Tricuspid aortic valve } \\ \text { TGA } & \text { Thermogravimetric analysis } \\ \text { UV } & \text { Ultraviolet }\end{array}$




\section{Chapter 1: Introduction}

Cardiovascular disease is a leading cause of death in Canada that kills approximately $23 \%$ of those affected, mostly men (Government of Canada, 2016; Moore \& Jimenez, 2011). The ascending thoracic aortic aneurysm (ATAA) is the most common pathology of the left ventricle outflow track, which has an incidence of approximately 10.4 per 100,000 person-years (Clouse et al., 1998). The ascending thoracic aorta (ATA) is deemed aneurysmal if the maximum diameter is $50 \%$ greater than the average healthy size ( $25 \mathrm{~mm}$ ) (Rosamond, Flegal, Friday, Furie, \& Go, 2007) corresponding approximately to 50mm for an adult. This disease involves the aortic root and/or ascending aorta in approximately $60 \%$ of the cases (Isselbacher, 2005; Kouchoukos \& Dougenis, 1997) (Figure 1). It is caused by a remodeling of the medial layer of the vessel that produces an irreversible dilation of the vessel, resulting in an increased risk of vessel rupture or dissection due to weakness of the aortic wall. Untreated, rupture or dissection carry a mortality rate of $80-90 \%$.

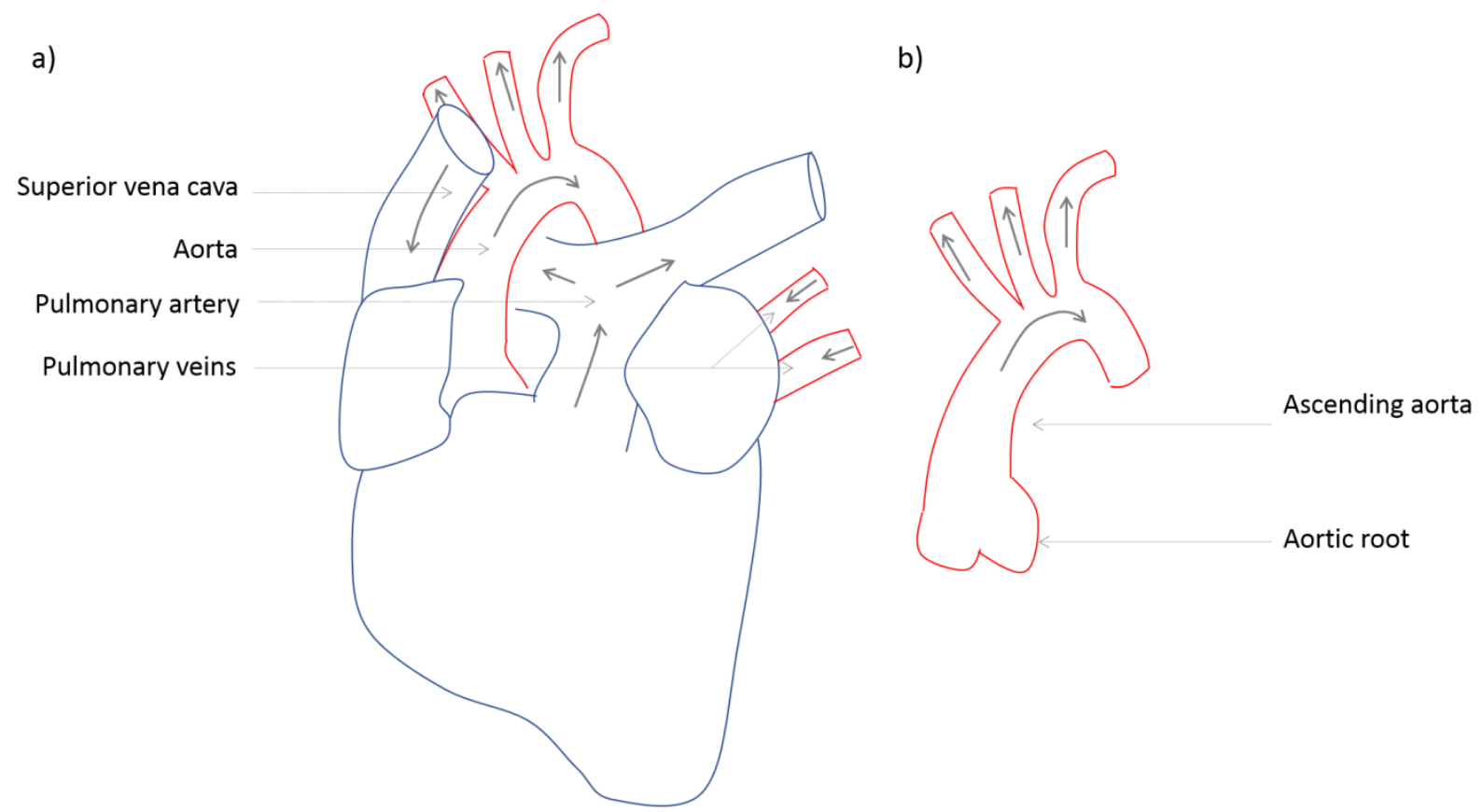

Figure 1. a) Typical geometries of a human heart and b) an aorta with the blood flow directions illustrated with grey arrows.

The treatment of an aneurysm involves cardiac surgery to replace the ATAA with a synthetic graft. Such operations when performed under emergency conditions following rupture or dissection have a surgical mortality of $15-24 \%$. Elective replacement prior to rupture or dissection is much safer with an estimated surgical mortality of 3.4\% (Rampoldi et al., 2007; Williams et al., 2012). Such medical procedures require 
high-levels of competency and precision that need to be acquired with significant training. To aid in acquiring the skills needed to perform complex procedures such as cardiac surgery, simulations are often used. Simulations are increasingly used to teach skills in a safe and controlled environment during interactive and/or immersive activities with actions or scenarios mirroring real life. They also allow trainees to practice several times on numerous and different models. A large gain in performance has been found after simulation training (Norman, Dore, \& Grierson, 2012) when the training tools are appropriately chosen for the tasks and level of experience of the learners (Adams et al., 2015; Maran \& Glavin, 2003; Munshi, Lababidi, \& Alyousef, 2015). Moreover, many studies have proven that medical simulations improve the ability to learn rapidly (Rosen, 2008), increase the knowledge, confidence and management skills (Chakravarthy et al., 2011).

Simulations used for open cardiac surgical procedures usually involve an idealized phantom (also known as simulator) composed of commercial tissue substitutes or expensive single and cadaveric models. However, current phantoms lack patient specific pathologies and are often crude tubular models. Their mechanical properties are also very unrealistic, moreover none of the present models can be perfused in a physiologic manner. There is, therefore, a need for realistic physical training simulators to help surgeons to practice techniques thus improve the procedures and patient safety.

3D printing is a very promising and burgeoning field in medicine. It is an additive manufacturing process in which several layers of monomer liquid can be laid down and solidified through ultraviolet rays or filaments can be melted by heat to make 3D objects in almost any shape. Powders are also an option but will not be used in this study. The resulting materials may be rigid or flexible. This 3D technology is well suited to improving surgical training phantoms especially if one can create patient specific models which replicate the actual normal and pathological tissue characteristics and patient-specific geometries. A most important aspect of 3D printed models used in surgical training relate to their material properties. To date, the simulators available lack the material properties needed to simulate surgical and the complex (non-uniform, heterogeneous, anisotropic) properties of ascending aortic tissue. The novel aspect of this thesis is to focus on the creation of synthetic composite materials which mimic the biomechanics of ex vivo/ in vivo tissue and allow for the full range of surgical manipulation under life-like conditions. 


\section{Chapter 2: Objectives, research question and overview of the thesis}

\subsection{Objectives and research question}

The goal of my thesis research is to improve the surgical training of cardiac surgeons by developing better surgical training models. In particular, my research focused on using 3D printing of material composites to create normal and pathologic aortic geometries with similar aortic material properties which would be suited for perfusion, resection, cutting and suturing. Passive ex vivo and in vivo biomechanical characteristics of human thoracic ascending aortic tissues (ATA, ATAA) were measured and used to guide and validate material selection and composite creation.

My fundamental research question was : Can 3D printed material be used to represent human aortic tissue needed for physical simulation?

The objectives were to:

1. Create a composite material by combining materials compatible with $3 \mathrm{D}$ printing to simulate the aortic tissue mechanical properties (ATA, ATAA):

1.1. Design and create 3D printed composites made of available 3D printed materials,

1.2. Quantify the material properties of the composites, the aortic tissue and commercially available materials used in medical simulation by mechanical testing for comparison purposes:

1.2.1. Tensile testing,

1.2.2. Surface hardness,

1.2.3. Suture retention strength.

2. Create a series of anatomically correct ATA and ATAA training phantoms:

2.1. Static rigid phantom,

2.2. Static mechanically realistic phantom,

2.3. Physiologically realistic phantom.

3. Evaluate the ability of 3D printed composite aortic models to replicate in vivo physiological tissue dynamics. 


\subsection{Overview of the thesis}

This thesis comprises nine chapters. The introduction is in Chapter 1, and the objectives as well as the research question are discussed in Chapter 2.

Chapter 3 presents a literature review pertinent to the thesis and contains exerts of a paper I co-authored with Alexander Emmott (Emmott et al., 2016). It explains the biomechanics of ATA and their constitutive frameworks.

Chapter 4 is a published manuscript that reviews the use of 3D printing in medical education and the state of the art of the current technologies. A method to create patient-specific simulator based on the example of an aortic phantom was developed and explained step-by-step.

In Chapter 5, a second published manuscript is presented that focuses on designing and testing tissuemimicking multi-material composites for 3D printing. The mechanical properties of the material were evaluated and compared to normal and aneurysmal aortic tissues (ATA, ATAA).

In Chapter 6, a draft manuscript that tested 3D printed aortic models in a flow loop for physiological fidelity is presented. In this work, we compared their echo-properties to the ones obtained for patients in the MUHC aortic clinic.

Finally, conclusions and recommendations are presented in chapter 7 along with the original contributions to knowledge (Chapter 8 ) and the future work in Chapter $9 .$. 


\section{Chapter 3: Literature review}

\subsection{Biomechanics of the thoracic ascending aorta}

\subsubsection{Thoracic ascending aorta}

Aneurysms of the thoracic ascending aorta often require prophylactic surgical intervention to resect and replace the aortic wall with a synthetic graft to avoid the risk of dissection or rupture. Such a procedure is recommended for patients with a maximum ascending aortic diameters at a range of $4.2-5.5 \mathrm{~cm}$, depending on etiology and need for concomitant cardiac surgery (Bonow, 2008; Boodhwani et al., 2014; Hiratzka et al., 2010; Kuzmik, Sang, \& Elefteriades, 2012). Although the risk of dissection and rupture increases with the size of aneurysm, different pathologies, including aortic valve phenotype and connective tissue disorders uniquely influence the mechanical dysfunction of the aortic wall. Dissection and rupture are mechanical modes of failure caused by an inability of the tissue to withstand local tissue stresses.

The cause of ascending ATAA is often considered idiopathic, however, several pathological features have been suggested. This pathology is associated with known genetic mutations (Marfan syndrome, Ehlers Danlos syndrome) and bicuspid aortic valve patients. Approximately $60 \%$ of thoracic aortic aneurysms involve the aortic root and/or ascending aorta (Isselbacher, 2005; Kouchoukos \& Dougenis, 1997).

\subsubsection{Characteristics of the ascending aortic tissue}

Aortas have a three-layer structure that varies with location and age. The inner layer that comes in contact with blood flow is the intima, while the adventitia is the outer fibrous layer. The media (middle layer) is usually the thickest layer and provides the majority of biomechanical response in healthy tissue. The passive elastic behavior and tensile strength of the vessels are mostly controlled by the fibrils of elastin and collagen (Lasheras, 2007; Tsamis, Krawiec, \& Vorp, 2013) both largely present in the media (Figure 2). At $80 \%$ of the total thickness of the aortic wall, the media is also the largest layer of the tissue (Morton \& Barnes, 1982). Collagen fibers are mostly arranged circumferentially (Wolinsky \& Glagov, 1964) and their orientations are important to the mechanical properties of the tissue as well as their directional dependency (isotropy, anisotropy) (Gasser et al., 2012). Normal aortas have been reported to be anisotropic with increased stiffness in the circumferential directions (Holzapfel, Sommer, \& Regitnig, 2004; Mulvany \& Aalkjaer, 1990). 

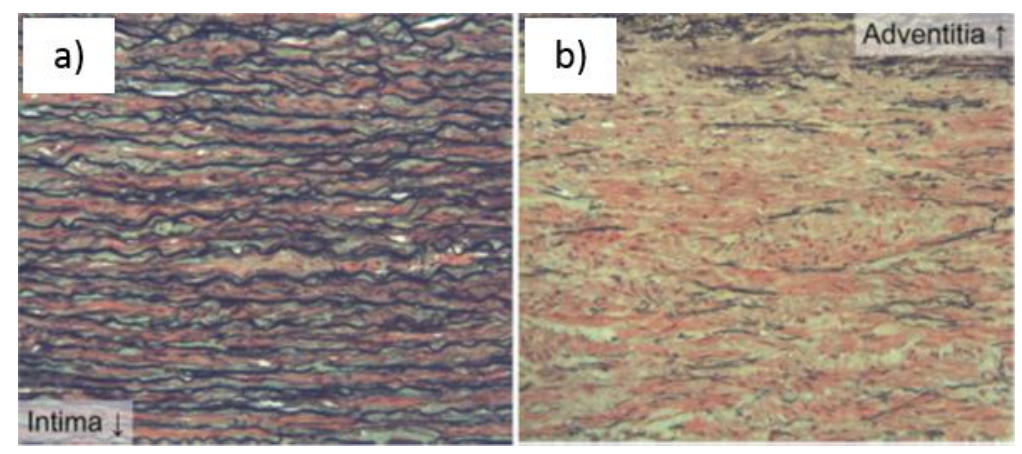

Figure 2. Histopathology of the ascending aorta using Movat pentachrome staining, a) in a 52-year-old man with a nondilated ascending aorta and b) a 51-year-old man with a 5.8-cm dilated ascending thoracic aortic aneurysm. Black indicates elastin; red/purple, smooth muscle cells; blue, mucopolysaccharides; and yellow, collagens.

Reprinted with permission (Emmott et al., 2016).

A mechanically-normal aorta distributes blood flow from the heart to the capillaries in order to carry oxygen to the body. This portion of the cardiovascular system is also known as the systemic circulation in which arteries have non-linear viscoelastic material properties and thus time-dependent deformations. These biomechanical properties are governed by both the composition and structure of the vessel wall. The elasticity aids to the propulsion of the blood during the loading cycles, when expansions and contractions of the arteries are generating energy. The energy will be absorbed by the tissue and its amount would vary with the patient health conditions (Chung, Lachapelle, Cartier, Mongrain, \& Leask, 2017). For instance, Chung et al. (2014) have shown that a loss of energy correlates with the aneurysm size and histopathologic findings. Such parameters could, therefore, be used as predictors for risk assessment.

Under load free conditions, residual stresses and strains are present in the tissue. When an artery is radially cut, the ring unrolls and opens with an opening angle that is dependent on the residual stress and heterogeneity of the vessel (Taber \& Humphrey, 2001). This residual stress in the artery is necessary for the active expansion and contraction that most vessels can control to modulate physiology (Humphrey, 2010; Taber \& Humphrey, 2001).

\subsubsection{Alteration of the mechanical properties}

Mechanical properties of the aorta can be affected by different factors such as ageing, diseases (atherosclerosis, diabetes, aneurysms, hypertension) and/or quality of life (smoking, exercise, food). Ageing causes many biomechanical changes to vessels including tissue remodeling due to degradations and syntheses of the tissue (M. Wang \& Lakatta, 2002), an increase of the stiffness and stress due to heart 
rate and blood pressure (Länne, Sonesson, Bergqvist, Bengtsson, \& Gustafsson, 1992). It also affects the amount of collagen and concentration of elastin that increases and decreases respectively. Fibers of elastin tend to fragment (Tsamis et al., 2013) but the mechanisms behind this degeneration remain unknown. The wall thickness of ageing aortas has been reported to either increase (A. E. Li et al., 2004) or remain constant (Turkbey et al., 2014) over time. Even remaining constant requires the vessel to remodel and synthesis new material to keep up with the expansion. Mechanical fatigue failure likely also contributes to this tissue remodeling over a lifetime (Greenwald, 2007). As a result, the aorta naturally dilates over time at a rate of approximately $0.15 \mathrm{~mm}$ per year (Hager et al., 2002; Turkbey et al., 2014).

When an aneurysm occurs in the ascending aorta the geometry and biomechanics of the tissue change (Driessen, Peters, Huyghe, Bouten, \& Baaijens, 2003). Generally degenerative ATAA has a decrease of the quantity and quality of elastin fibers which stiffen the tissue (Iliopoulos, Kritharis, Giagini, Papadodima, \& Sokolis, 2009). In older patients, a loss of smooth muscle cells and increase in collagen content further increases the tissue stiffness. Over the evolution of the disease, the loss of tissue structure results in material properties that are not dependent on the direction (isotropy) (Chung et al., 2017). Aneurysms have been attributed to medial degeneration (formerly termed cystic medial necrosis or cystic medial degeneration (Hirst, Johns, \& Kime, 1958)), a non-inflammatory loss of smooth muscle cells and fragmentation of the elastic fibres (Carlson, Lillehei, \& Edwards, 1970; Hirst et al., 1958). Medial degeneration that leads to aneurysm is believed to be an imbalance in the synthesis and degradation of the medial matrix proteins but, currently, the cause of medial degeneration is still poorly understood. What is clear is that, as the ascending aorta dilates, it continues to lay down new tissue and tries to preserve its thickness, which would otherwise decrease with increasing diameter (Choudhury et al., 2009; Tang et al., 2005). Although medial thickness might decrease with idiopathic aneurysm (Tang et al., 2005), total wall thickness usually remains constant (Choudhury et al., 2009; Tremblay et al., 2009). The effect of age on aortic wall thickness is less clear and dependent on the disease state. In fast growing aneurysms, the rate of synthesis or degradation can cause the vessel to thin.

\subsubsection{Characterization of biomechanical parameters defining the aorta}

\section{Laplace's law}

Aortic size as a predictor of mechanical stability. Maximal aortic diameter is the primary criterion for patients who undergo prophylactic surgery of the aorta with graft replacement. It is important to acknowledge that this measure is not a mechanical property, but instead acts as a surrogate for tissue 
circumferential stress $\left(\sigma_{\theta}\right)$. This mechanical interpretation follows Laplace's law, which states that the circumferential stress in a vessel simplified by a cylinder with a defined thin wall thickness $(\tau)$ is a function of the aortic radius ( $r$ ) and the pressure exerted on the vessel wall (P) by the following relation (Figure 3):

$\sigma_{\theta}=\frac{\operatorname{Pr}}{\tau}$

This simple model of tissue stress fundamentally assumes the vessel material is linearly elastic and has no directional dependency in its mechanical properties (isotropic). It also assumes the vessel is a uniform, straight cylinder with no other applied stresses other than blood pressure. In this model, the circumferential stress in the vessel wall is then proportional to the size of the aneurysm alone if the tissue thickness and blood pressure remain constant. This suggests that, at exceedingly large aortic diameters, the circumferential stress might exceed the ultimate tensile strength of the tissue and rupture might occur. Setting a threshold diameter limit implicitly assumes that all thoracic aortas have the same ultimate strength, thickness, and blood pressure.

The derivation of Laplace's law assumes that the stress-strain relationship is linear, and related by a constant tissue stiffness modulus, also known as the Young's or elastic modulus (E). Although aortic tissue is nonlinear and hyper-elastic, the linear elastic model can describe tissue deformation for small changes in strain (see green linear curve on Figure 4). The equation for linear stress-strain is presented as follows:

$\sigma=$ E. $\varepsilon$

where $\sigma$ and $\varepsilon$ are the material stress and strain, respectively. It is well known that vessel stiffness (E) is variable from patient to patient and generally increases with age (Okamoto et al., 2002; Okamoto, $\mathrm{Xu}$, Kouchoukos, Moon, \& Sundt, 2003) and with aneurysm (Choudhury et al., 2009; Vorp et al., 2003). In a stiff rather than a compliant vessel, it is intuitive that wall stress will increase at a much faster rate as the vessel expands. Even with the assumption of linear elasticity, the sole use of diameter neglects differences in tissue stiffness. 

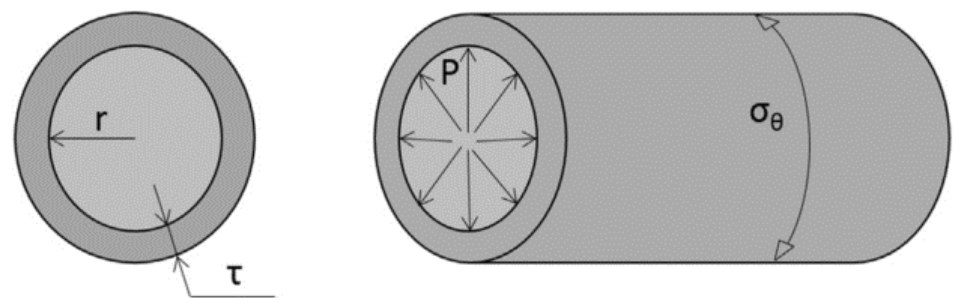

Figure 3. Illustration of Laplace's law: wall tensions in the vessel (simplified by a cylinder with a radius $r$ larger than a wall thickness $\tau$ ) when pressure $\mathbf{P}$ is exerted by the aortic blood flow. $\mathrm{P}$ is assumed evenly distributed over the wall. $\sigma \theta_{\theta}$ is the circumferential stress.

Reprinted with permission (Emmott et al., 2016).

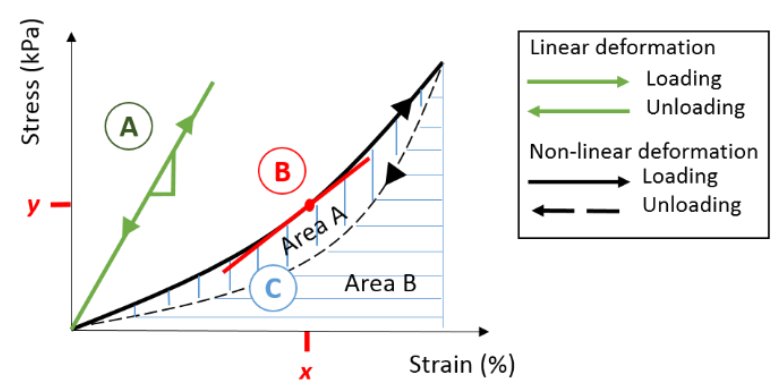
A E: Slope of the linear elastic deformation
(B) $\mathrm{E}_{\mathrm{m}}$ : Slope of the loading curve defined by a red tangent line at $\boldsymbol{x} \%$ strain or $\boldsymbol{y} \mathrm{kPa}$ stress
(C) Energy loss: $\frac{\text { Area } A}{\text { Area } A+\text { Area } B}$

Figure 4. Typical shape of stress-strain curve for linear elastic (green line) and visco-elastic response of biological tissue (black line). (A) Elastic modulus of a linear elastic material ( $E$ ), (B Elastic modulus of a nonlinear material $\left(E_{m}\right)$ and (C) Energy loss. Reprinted with permission (Emmott et al., 2016).

\section{Ex vivo evaluation}

\section{Measurement of the tissue biomechanics}

Ex vivo mechanical testing of excised ascending aorta is the "gold standard" for assessment of mechanical properties, such as tissue stiffness. Healthy aortic tissue is acquired from transplant donors or from autopsy and ascending ATA specimens are acquired during elective surgical repair. Mechanical testing is done within 24-48 hours and specimens are kept mostly in a refrigerator (Choudhury et al., 2009; E. D. Matsumoto, Hamstra, Radomski, \& Cusimano, 2002; J. S. Matsumoto et al., 2015), a freezer (Pham, Martin, Elefteriades, \& Sun, 2013), or on ice (Chung et al., 2014). Freshness of the sample and techniques to keep the structure intact are major issues. To avoid tissue degeneration, aortas are often kept in saline 
or gauze at a low temperature. Before testing an equilibration at room temperature is required for the tissue. Ideally, because of the temperature dependence of material properties, tests are conducted at body temperature.

Although ex vivo mechanical testing is rigorous and reproducible, there are important limitations. Most importantly, it is a post-operative or post-mortem analysis, because it requires the tissue to be removed from the patient. It is also labour-intensive which translates to a limited number of tissue samples in any given study. Thus, interpretations and the conclusions might not be representative of the larger patient population. As can be seen in Emmott et al. (2016), the number of samples ranges between 6 and 40 in tensile testing, which leaves very little room for patient subcategorization with any statistical power. Also, ATA tissue is inhomogeneous with regional variations (Choudhury et al., 2009; Chung et al., 2014; Iliopoulos et al., 2009). Most studies neglect this potential variability especially when a small quantity of tissue is analyzed. Moreover, several tissue samples are sometimes extracted from the same subject, thus the diversity of the patient population and their tissue characteristics remain limited (Khanafer et al., 2011; T. Matsumoto et al., 2009). This can be overcome by systematically sampling labelled quadrants of ascending aorta for each specimen (Choudhury et al., 2009; Tremblay \& Leask, 2011).

\section{Tensile testing derived metrics}

The passive biomechanical properties of the aorta are often evaluated using ex vivo tensile testing in which a sample of aortic tissue is stretched and the corresponding tension in the wall is measured. By measuring this tension along with the amount of stretch (displacement), a stress-strain relationship curve is developed. Vascular tissues, like the ascending thoracic aorta, are hyper-elastic materials characterized by a nonlinear relation between stress and strain (Figure 4). Moreover, vascular tissue is viscoelastic, which results in hysteresis in the tensile testing loop where the stress-strain relation follows different paths when load (loading) and removing load (unloading) are applied. These tensile tests can be conducted in a single direction, usually in either a circumferential or longitudinal orientation, and this is called uniaxial tensile testing; or tests can be conducted simultaneously in the circumferential and longitudinal directions, and this is called biaxial tensile testing. For uniaxial tensile testing, samples are often cut in rectangular shapes oriented along the desired axis and stretched lengthwise. Uniaxial testing generalizes the behaviour of the sample with 2 independent pieces, which might have unique structural imperfections and geometrical parameters, and can increase analytical errors. It also neglects the transfer of stress from one direction to the other. Therefore, biaxial tensile testing, which takes into consideration 
the tensile properties in 2 axes simultaneously, is recommended. In this configuration, specimens are cut in squares, which allows for testing in 2 perpendicular axes and then stretched to obtain the tissue's stress-strain response. Equi-biaxial tensile tests are biaxial tests in which the tissue is stretched or loaded simultaneously and equally in both axes. This allows for the coupled response of the tissue in both directions to be measured.

Currently, there are several families of testing protocols that are used to evaluate the mechanical state of vascular tissues. Herein, we focus on the cumulative understanding of the mechanics of ascending aortic tissue using planar tensile testing; however, it is important to acknowledge that other metrics are needed for a holistic understanding of tissue biomechanics. In interpretation of published tensile test data, it is important to note there are multiple definitions of stress and strain or stretch that can be used. However, the main biomechanical parameters evaluated in the literature are the following.

The apparent elastic modulus is a parameter of a non-Hookean material (a material with a nonlinear stress-strain relation) and can be thought of as a material's resistance to deformation at a given strain or stress value. Elastic modulus, therefore, corresponds to the slope of the stress-strain curve under loading (Figure 4) and, because it is variable along the curve, must be defined at a specific strain or stress value. Although general trends can be derived from a study to study comparison (for instance, ascending ATAA are generally much stiffer than healthy ATA), stiffness values cannot readily be compared unless they are reported with a similar definition of stress and strain and reported at the same point on the curve. Notably, a material's stiffness describes its relationship between the applied load (stress) and tissue stretch (strain) but does not describe the material strength. Because of the nonlinear stress-strain relationship of biological tissues, stiffness is a relative metric and compliant materials (low stiffness) might be strong and rigid materials (high stiffness) might be weak.

Energy loss is a recently proposed biomechanical parameter. It has the advantage of being an integral metric of the mechanical response to loading and unloading and is self-normalizing, which makes it less susceptible to strain and stress definitions and testing variables. It is a measure of the relative amount of energy loss of the loading cycles during tensile testing (Figure 4) (Chung et al., 2014). This reflects the aorta's natural physiologic function in absorbing energy during systole and returning a proportion during diastole. Increasing levels of energy loss indicates poor efficiency in performing this function. Higher 
energy loss has been found to be associated with aortic size and to reflect the underlying histopathology (Chung et al., 2014).

\section{Indentation derived metrics}

Indentation testing, also known as a compression test, is used to evaluate the local material properties in contrast to tensile testing designed for macroscopic properties. It characterizes a large panel of tissue including the vascular tissue (T. Matsumoto, Goto, Furukawa, \& Sato, 2004) but techniques are often custom-made thus difficult to compare. Moreover, publications on the evaluation of human aortas remain rare as porcine aortas are easier to collect and test.

Indentation can be used to characterize the compressive stiffness of the material with a compressive modulus $\left(E_{\text {comp }}\right.$ ) generally defined by the method of Oliver and Pharr (1992). It helps to determine the tactile properties of the materials thus the haptics on a macro level. The parameter is calculated from the Poisson's ratio of the material, the apparent elastic modulus and Poisson's ratio of the indenter ( $\left.E_{\text {ind }}, v_{\text {ind }}\right)$, as well as the reduced modulus $\mathrm{E}_{\mathrm{r}}$.

$E_{\text {comp }}=\frac{\left(1-v^{2}\right) E_{\text {ind }} E_{r}}{E_{\text {ind }}-E_{r}\left(1-v_{\text {ind }}^{2}\right)}$

The $E_{r}$ is derived from the material stiffness obtained from compression $\left(S_{i}\right)$ and projected contact area $\left(A_{c}\right)$ of the spherical tip. $A_{c}$ is expressed with the radius of the sphere $(R)$, the contact depth $\left(h_{c}\right)$ computed with the maximum depth penetration $\left(\mathrm{h}_{\max }\right)$, the maximum load $\left(\mathrm{P}_{\max }\right)$ and stiffness corresponding to the slope of the beginning of the unloading curve (see Figure 5).

$E_{r}=\frac{\sqrt{\pi} S_{i}}{2 \sqrt{A_{c}}}$

$\mathrm{S}_{\mathrm{i}}=\frac{\mathrm{dP}}{\mathrm{dh}}$

$A_{c}=2 \pi R h_{c}$

$\mathrm{h}_{\mathrm{c}}=\mathrm{h}_{\max }-\frac{3 \mathrm{P}_{\max }}{4 \mathrm{~S}_{\mathrm{i}}}$ 


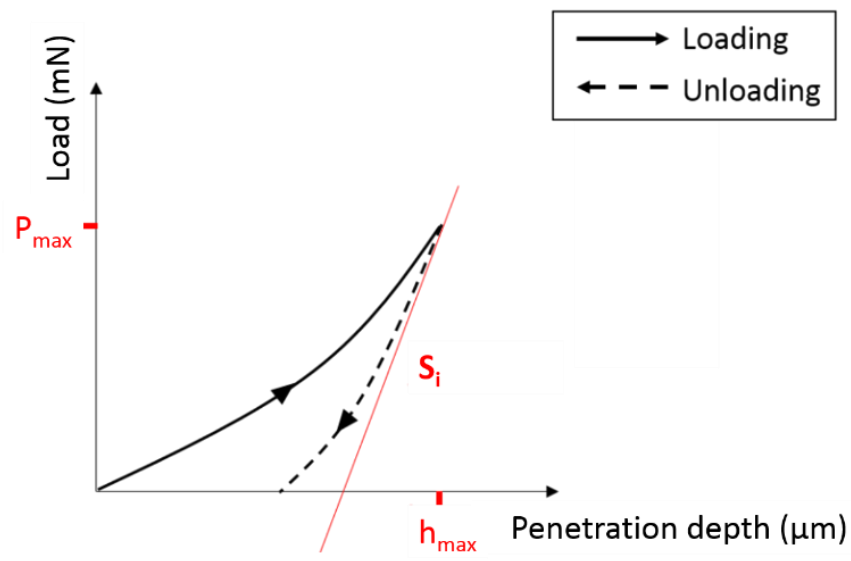

Figure 5. Typical loading and unloading curves of the load and penetration-depth with visco-elastic materials in indentation. $\mathrm{P}_{\max }$ is the maximum load, $\mathrm{h}_{\max }$ the maximum depth and $\mathrm{Si}$ the stiffness of the material calculated with the slope of the beginning of the unloading curve

\section{In vivo evaluation}

Clinical imaging modalities are the current standard for assessment of the degree of aortic dilation in cases of suspected aneurysm and aortic valve dysfunction. As a result, these methods have been adapted to infer the in vivo biomechanical properties of the aortic wall, in a preoperative and minimally invasive way. O’Rourke, Staessen, Vlachopoulos, and Duprez (2002) provide a comprehensive summary of the metrics used for in vivo mechanical imaging. In most cases, a patient with suspected aortic disease will undergo echocardiography (echo) imaging to observe valve function and phenotype and to measure the dimensions of the vessel. Echo Doppler measurements have the added benefit of visualization of hemodynamics in the ascending aorta, including the jet/wall interaction during systole. Two-dimensional and $\mathrm{M}$-mode echo measurements in tandem with an aortic pressure line can be used to determine a pressure-diameter relation (often only in the circumferential direction), which can reveal the level of aortic distensibility and estimate stiffness. Using diameter change, in an observation of the long-axis of the aorta, Baumgartner et al. (2005) showed that young patients with Marfan Syndrome had significantly stiffer aortas compared with those in control subjects (Baumgartner et al., 2005). Similar work has been used to reveal the in vivo stiffening behaviour of the ascending aorta in response to chronic hypertension (Vitarelli et al., 2010). Recalling Hook's law, strain alone is insufficient to estimate the stress in the tissue and requires a priori knowledge of the tissue material properties. Transverse (or short-axis) imaging of the aorta using 2- dimensional echo with speckle tracking, a process that tracks the movement of natural acoustic markers ("speckles") that move with the wall, can better account for circumferential diameter change. Using this method, which is well established for left ventricle strain assessment (Hoit, 2011), the 
full circumference of the aorta can be imaged, which accounts for nonuniform wall motion (Teixeira et al., 2012). New developments in 4-dimensional (4D) echo (3-dimensional reconstruction with temporal resolution) will likely provide a more robust analytical tool for in vivo mechanical measurements and allow for simultaneous strain assessment in the circumferential and longitudinal aortic axes. Again, for such in vivo strain imaging to be used to estimate tissue stress requires knowledge of the tissue's mechanical properties. Strain imaging will require extensive population analysis and comparison with ex vivo mechanical properties to become a reliable tool for patient stratification.

\subsection{Surgical education}

\subsubsection{Medical simulation: an increasingly popular educational method}

\section{Concept of simulation}

Surgeons require a high skill set that is traditionally acquired through an intensive training in the clinic which, for several years now, include medical simulation. This educational method allows a repeatedly practice at any time in safe and controlled environments without harming patients (de Montbrun \& MacRae, 2012; Dutta \& Krummel, 2006). The effectiveness of the training as well as its significant improvements in the learning curves of the users have been demonstrated in the literature (Chakravarthy et al., 2011; de Montbrun \& MacRae, 2012; Dutta \& Krummel, 2006; Marr et al., 2012). The main advantage of simulation is to provide an experience outside the clinical environment. Since the amount of experience is generally tied to the development of competency, the ability to continually practice and be engaged with simulated cases would greatly help the development of expert surgeons.

Simulations are virtual or physical but can be complimentary. However, physical interaction has been proven to be the key for learning (Dutta \& Krummel, 2006) and gaining motor skills needed for surgical intervention (Armillotta et al., 2007; Kalejs \& von Segesser, 2009; Sulaiman et al., 2008; Suzuki et al., 2004). The importance of being trained with graspable models was highlighted by many studies (Lujan \& DiCarlo, 2006; Shah, Ahmed, Shenoy, \& Srikant, 2017) who found that students had a preference for kinesthetic learning, also known as tactile learning. It teaches through interactions, physical involvement by the manipulation of tools made of textual materials mimicking real tissues. The importance of touch as well as real like tissue manipulation for learning and surgical training is the major impetus to develop a large panel of custom-made physical aortic simulators for the future generation of cardiac surgeons. The 
models would have the advantages the anatomy of the vessel (normal or pathologic) but would also allow the practice of several medical manipulations (instrument handling, knot tying, suturing, sewing).

Three levels are generally distinguished. Firstly, there is a technical goal which may be done without any clinical context. Trainees will be just learning and practicing the procedure. Secondly, there is the contextual approach where technical training is done in conjunction with cognitive simulation in which typical scenarios may be reviewed and discussed. And thirdly, there is a full blown scenario providing cognitive and circumstantial contexts (Dutta \& Krummel, 2006; Evgeniou \& Loizou, 2013) in which the trainee must manage a procedure in a hands on approach. The stress and anxiety levels generally increase through the steps and are known to be important as they will prepare the students to unpredictable reallife situations (Kneebone \& Baillie, 2008).

Several categories of training were identified in the literature such as the (Chakravarthy et al., 2011; de Montbrun \& MacRae, 2012; Dutta \& Krummel, 2006; Marr et al., 2012):

- $\quad$ standardized patients (played by actors),

- partial-task trainers: trainers for specific medical procedures,

- mannequins: patient simulators,

- screen-based computer simulators: virtual patients on screen,

- virtual-reality simulators: virtual patient's organs and anatomy on screen.

However, none of the methods would be enough by themselves as they would be better used together and in conjunction (Fox, Walker, \& Draycott, 2011).

\section{Simulators}

Human cadaveric tissue is commonly used in surgical education for the representative anatomically correct geometries (anatomic fidelity). However, types (disease, congenital defect for instance) and numbers of pathologies, as well as donors, would not allow every single student to practice on deceased patients. Post-mortem tissue left in the bodies or collected from them might also present alterations of properties depending on the type of preservation. We therefore have a "pseudo tissue fidelity" with mechanical properties varying among cadavers.

Animal tissue is also an alternative way to develop surgical skills but could differ in anatomy and properties. Therefore, both methods would give the opportunity to replicate surgeries and develop motor 
skills, even though they would present limitations, remain expensive and impractical for large scale repeated training.

Developing skills, knowledge and experience while being confronted to a large panel of pathologies with realistic tactile feel is primordial for optimal training. For those reasons, synthetic simulators were developed to compensate the lack of living tissues and organs. Needs would differ depending on the medical field and specialization, but the concepts would remain mostly the same for every application. One of the biggest challenges today in surgical simulation is the lack of materials to develop deformable polymers that would imitate the characteristics of soft tissue with a representative anisotropy/ isotropy, viscoelasticity and compressibility (Zhang, Zhong, \& Gu, 2018). In addition, it has been shown that a good training tool should present specific features such as the possibility to use models several times, its feedback while practicing if instructors are not supervising, and variation of the level of difficulties with clinical variations (Evgeniou \& Loizou, 2013). Creating surgical simulators is therefore a complex task and a limited number of applications and/or characteristics would have to be defined at the very beginning of the process.

\section{Bipolar definition of the fidelity}

The bipolar concept of low-fidelity (LF) and high-fidelity (HF) is common in medical simulation even though the definition of fidelity remains unclear. However, it usually refers to the degree of realism of the simulator (Paige \& Morin, 2013), thus the physical resemblance to human patients (how the simulator looks, feels and acts) (Hamstra, Brydges, Hatala, Zendejas, \& Cook, 2014).

LF reproduces basic actions or scenarios but neglect factors and/or distractions (patient movements or discomfort) that the participants might experience under real-life conditions (Kneebone \& Baillie, 2008; Maran \& Glavin, 2003) and the training courses focus on small concepts and specific tasks until skills are fully mastered by the learners (sutures). However depending on the context, models should not be too oversimplified as they may affect skill transfers to the real tasks (Evgeniou \& Loizou, 2013). Medical professionals usually start with LF environments and move towards using HF for advanced training because complex skills require complex tools (Norman et al., 2012). HF simulation mimics real-life conditions thus recreate very realistic situations where trainees are subjected to any number of situations and vast arrays of pathologies without putting patients at risk. 
However, increasing the level of fidelity with complex and sophisticated simulators might not produce significant improvements. In pediatric emergency care training, it has been shown that increasing the level of fidelity has no impact on the learning of non-technical skills (practice-based training) or mental strain. HF did reduce the numbers of interruptions during the tasks and increased the performance (time between the beginning of the evaluation and oxygen prescription) (Meurling et al., 2014). In contrast, other studies (Adams et al., 2015; De Giovanni, Roberts, \& Norman, 2009; Lee, Grantham, \& Boyd, 2008) and review (Norman et al., 2012) suggest that there was no performance improvement using HF over LF, therefore complex HF might be superfluous especially for novices (Adams et al., 2015; Munshi et al., 2015) and when the costs are higher due to only small improvements and/or new features on the models (Maran \& Glavin, 2003).

To conclude, simulators need to be chosen in accordance with the tasks and learners (skills and amount of practice required to develop one of them (Evgeniou \& Loizou, 2013)) and LF simulation remains the most cost-effective method (E. D. Matsumoto et al., 2002).

\section{Typologies and dimensions of fidelity}

It may be too simplistic to limit the characterization of the simulation to LF and HF. A simulator could be both depending on which features (auditory, tactile or functional) are emphasized, in what contexts they are used and the learners being trained (Hamstra et al., 2014). For these reasons, Hamstra et al. (2014) identified two concepts: the structural fidelity (Does the simulator look like human patients?) and functional fidelity (What clinical tasks can we teach with the simulator?). Structural fidelity may not be the parameter that would improve the learning of the trainees since the physical resemblance does not necessarily affect the educational effectiveness. However, functional fidelity remains essential in surgical education as it would allow the practice of basic medical manipulations (suturing skills) with simulators mimicking the dynamic and tissue mechanics of real patients. Paige and Morin (2013) distinguished the physical, psychological and conceptual dimensions in simulation. The physical aspect defines the equipment (manikins) and environment (sounds, visuals, smells, etc.), when psychological refers to the emotional responses to the actions and the conceptual dimension recreates responses to medical situations such as a drop-in blood combined with a reduction in pulse strength for a state of shock. It is important for clinicians to learn the relationships between the different symptoms for good diagnostics. 
Norman et al. (2012) differentiated the psychological fidelity to the engineering fidelity. Psychological fidelity would be the most important dimension in simulation as even simple models with no complex mechanisms could be good enough for teaching. For instance, practicing suturing skills would lead to a better performance if the trainees use extracted porcine tissue than good visual computer-based simulations with bad haptic rendering. The level of fidelity remains a debate within the medical community as fidelity does not equal better learning necessarily. The differentiation could be done with the type of procedure one is learning and the aim of the simulation. Most studies have focused on basic skills and basic procedures (Cook et al., 2011) thus fidelity is not that important, and learning can be attained with simple models. However, most studies have not looked a complex procedure, such as cardiac surgery. It is generally thought, that the more complex the procedure, the more fidelity required. In addition, if one is using simulation as an adjunct to clinical training, then some parts can be learned on a basic trainer and the more complex parts learned in the clinical environment. If one is using simulation instead of clinical experience, then the simulation must have extreme fidelity.

\subsubsection{State-of-the-art of vascular phantoms}

Cardiovascular diseases are treated most of the time by endovascular or surgical interventions (Moore \& Jimenez, 2011). These cardiac procedures (listed in Table 1) require manipulations, reconstruction, repair or resection of diseased cardiac or vascular tissues which are all systematically involving the ATA (Rooke, Sullivan, \& Jaff, 2007; A. Wang \& Bashore, 2009).

A wide range of cardiovascular phantoms are available on the market, but their goals and characteristics differ from one another. An international study also revealed that there were large disparities between simulation centres as they would not necessarily use the same training tools for one specific task (Qayumi et al., 2014). Similarly, it was found that the education level of the participants, the lack of interprofessional experience, the curricular integration and the infrastructure support would differ around the world. Postgraduate students are more likely to have simulation classes involving online modulus and task trainers, regardless the speciality. In this section, the major commercial products for surgical simulation are presented. 
Table 1 Common cardiac diseases and treatments.

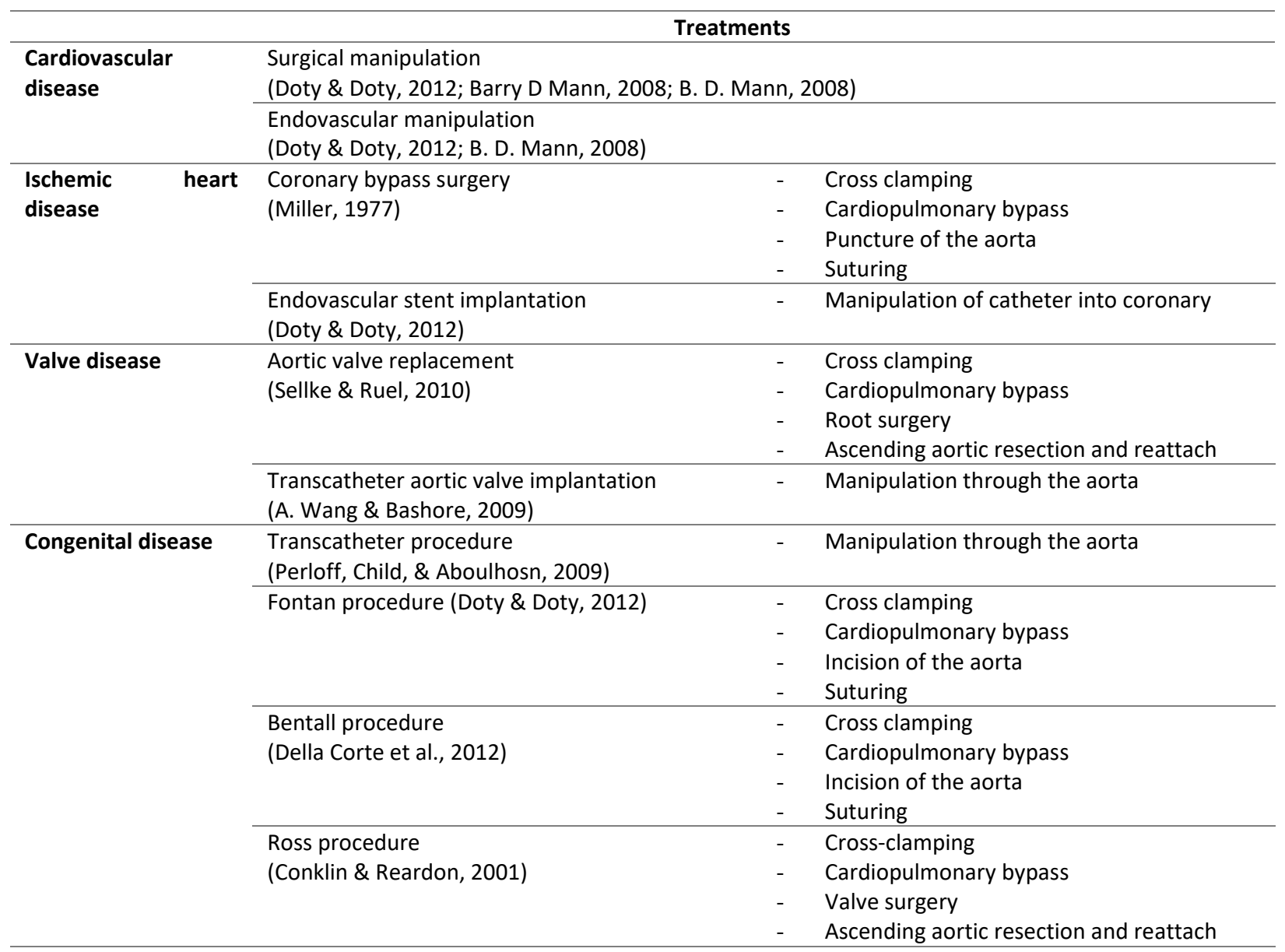

\section{Cardiac simulators for imaging}

Anatomically cardiovascular phantoms exist for medical image acquisition training. CAE Healthcare and Blue Phantom ${ }^{\text {TM }}$ collaborated to offer torsos suitable for transthoracic echocardiography ultrasound training. Similarly Computerized Imaging Reference Systems, Inc. has developed a phantom in which the organs are removable (Figure 6) (Blue Phantom, 2013; Computerized Imaging Reference Systems Inc., 2014). These are models for non-invasive training and although incorporate a simulated heart and aortic aneurysm, but they are not meant for surgical training. 
a)

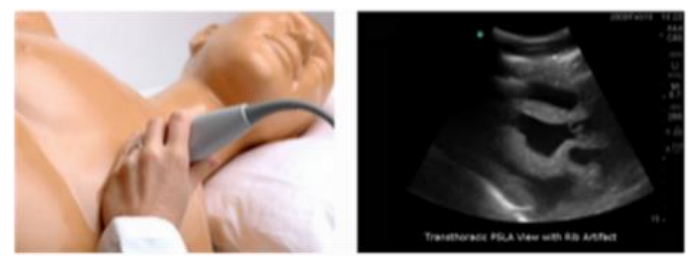

b)

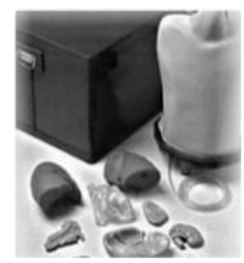

Figure 6. a) An ultrasound torso for medical image acquisition training (Blue Phantom, 2013) and b) an ultrasound torso with removable organs for transthoracic echocardiography ultrasound training (Computerized Imaging Reference Systems Inc., 2014).

These phantoms are an asset for evaluating medical imaging technologies but do not allow for physical manipulations. Boltz et al. (2010) described an anatomically correct beating heart phantom composed of soft silicone chambers and coronary arteries, which allows for additive pathologies (aberrant beats, stents, plaques, etc.) (Figure 7). The model is part of an assembly composed of the thorax, the compressor and the electrocardiography systems designed to evaluate technological advances in cardiac X-ray computed tomography.

Similarly, Presotto, Bettinardi, Petta, and Gilardi (2012) worked on a silicone left ventricle shaped in half ellipsoid, which contained water and allowed the application of specific pressures on its surface (Figure 7) to assess the effect of motion in cardiac positron emission tomography and single photon emission computed tomography studies. The aim of the phantom was to evaluate the quality of the positron emission tomography images in a beating heart context (Sipila, Teras, Kokki, \& Knuuti, 2007). The latter installed rubber balloons to represent the ventricle (inner balloon) and the cardiac muscle (gap between two balloons) of a dynamic heart with respiratory and cardiac movements. The phantom was filled with saline and computed tomography contrast agent (Figure 7). Silicone (Sylgard 527 A\&B ${ }^{\circledR}$ ) has also been used to design an assessment tool for magnetic resonance imaging with physiological motions of the heart (twist, compression and translation) (Kee et al., 2010; Yan, McDonough, Pilla, \& Xu, 2011)

An asymmetric left ventricular motion model that replicates various pathologies for ultrasound and cardiac magnetic resonance has been described (Tavakoli et al., 2012). This phantom is made of polyvinyl alcohol cryogel (PVA) and has latex balloons inserted for the heart's dilatations and contractions using air (Figure 7). The same material was used to create an atherosclerotic coronary artery composed of two layers in which a lipid pool was added for intravascular imaging and mechanical testing (Pazos, Mongrain, \& Tardif, 2010). 
a)

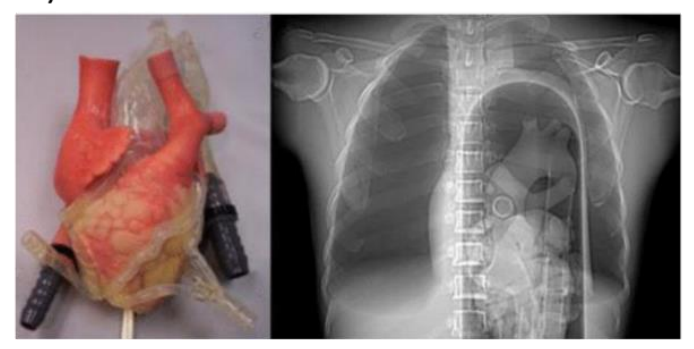

c)

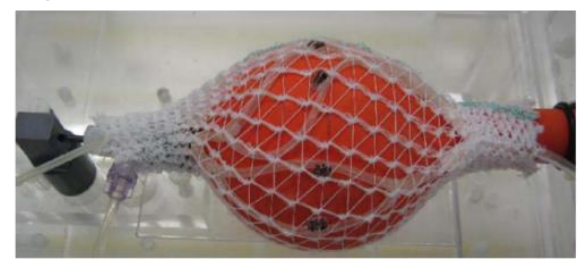

b)

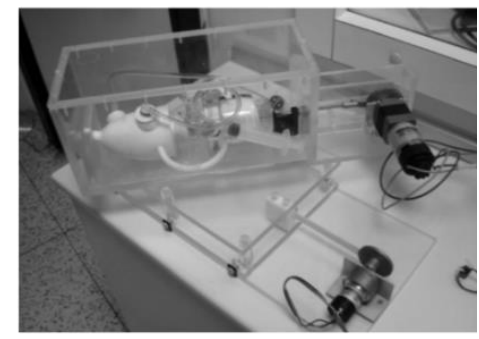

d)

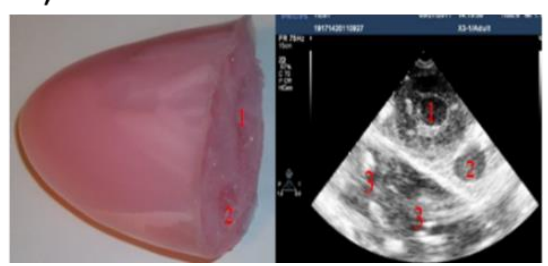

Figure 7. a) An anatomically correct heart silicone model for medical imaging technologies (Boltz et al., 2010), b) a nonanatomically correct left ventricle model made of silicone gum for cardiac positron emission tomography and single photon emission computed tomography (Presotto et al., 2012), c) a ventricle made of balloons for positron emission tomography (Sipila

et al., 2007) and d) a non-anatomically correct heart model made of polyvinyl alcohol cryogel for ultrasound and cardiac magnetic resonance (Tavakoli et al., 2012).

\section{Cardiac simulators for device testing}

Rapid prototyping has been used to create models for medical device testing. Biglino, Verschueren, Zegels, Taylor, and Schievano (2013) described a hypoplastic aorta and a right ventricle in Tangoplus Fullcure ${ }^{\circledR}$ elastomer (Figure 8). In the context of aortic stent design, Sulaiman et al. (2008) developed a life-size in vitro model of an aortic arch aneurysm made of soft and transparent silicone rubber, which allows circulation perfusion and visualization on each branch.

Our group has developed a multilayer model made of PVA and a lipid pool is mimicking deformable atherosclerotic coronary artery was developed (Pazos et al., 2010). The phantom is suitable for mechanical testing and intravascular ultrasound imaging for a plaque study (Figure 8).

Finally, a mock circulatory system made of polyurethane has been designed by Kolyva, Biglino, Pepper, and Khir (2012). This model is suitable was used to assess an intra-aortic balloon pump. The tool is composed of a left ventricle connected to a straightened aortic model with fourteen branches for the various peripheral organs. 
a)

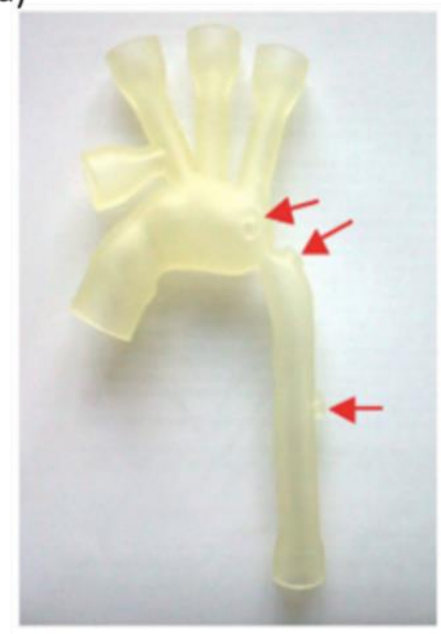

b)

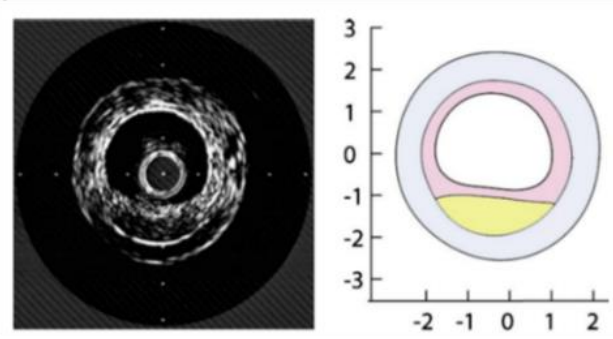

Figure 8. a) A hypoplastic aorta and a right ventricle printed in Tangoplus Fullcure ${ }^{\circledR}$ (Biglino et al., 2013) and b a) stenotic crosssection of a coronary polyvinyl alcohol cryogel phantom (image and geometry). Reprinted with permission (Pazos et al., 2010).

\section{Cardiac simulators for analyzing beating heart functions}

Training manikins for beating heart diagnosis are provided by companies like Koken Co., Laerdal Medical and Kyoto Kagaku Co. (Figure 9). As a matter of fact, personal health care workers can auscultate the models and differentiate several cardiac sounds (Koken Co., 2005; Kyoto Kagaku Co., 1999-2012). As an example the Cardiology patient simulator "K" of Kyoto Kagaku Co. can mimic eighty-eight pathologies. These models are not designed for surgical procedures.

a)

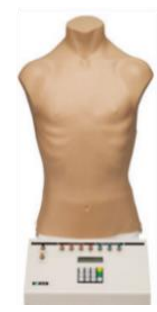

b)

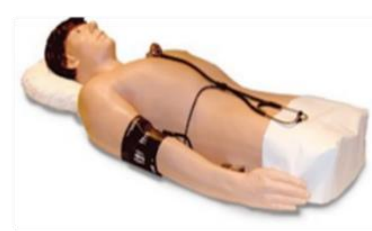

c)

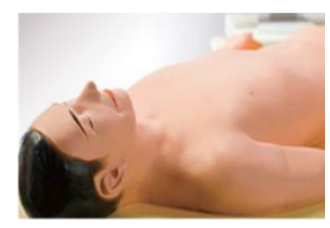

Figure 9. Physical assessment trainers with sounds of beating hearts: a) Koken Co.(Koken Co., 2005) b) Harvey ${ }^{\circledR}$ The Cardiopulmonary Patient Simulator with sounds of beating hearts, Laerdal Medical (Laerdal Medical, 2014), c) Cardiology patient simulator "K", Kyoto Kagaku Co. (Kyoto Kagaku Co., 1999-2012).

\section{Cardiac simulators for medical interventions}

Cardiovascular simulators for medical procedures can be divided in two groups. The "simple bench model" consists of practicing repeatedly specific tasks on simplified and non-expensive models. The "human 
performance simulator" has interactive phantoms, computer interfaces and high technologic systems in a realistic simulated environment for a complete surgery (Trehan, Kemp, \& Yang, 2014).

\section{"Simple bench model"}

Numerous manufacturers such as Lifelike Biotissue or The Chamberlain Group provide phantoms with training purpose. Nevertheless, realistic heart shaped simulators are limited. We found one example that was designed for dissection or removal of the left atrial appendage (Figure 10) (The Chamberlain Group, 2014a).

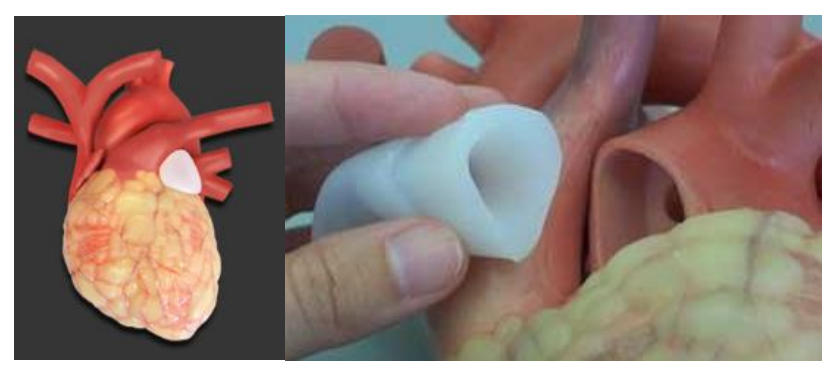

Figure 10. Heart phantom model for basic tasks (The Chamberlain Group, 2014a).

Hollow cylinders that may be filled with blood mimicking fluids are commercially available for mimicking aortas, vessels and coronary arteries. These tubes are somewhat suitable for practicing sutures, dissection repair anastomosis and cannulation (act of inserting a small tube) (Figure 11). Indeed, several training models are available, including valve implantation on aortic roots fixed to stands, catheterization through detailed transparent hearts or superimposed tissues that replicate arterial structures. Moreover, devices insertion (stent, balloon, wire) can be evaluated (Figure 11). 
a)

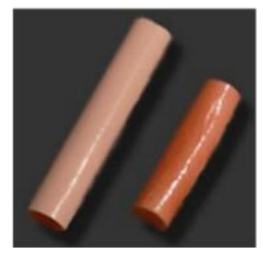

d)

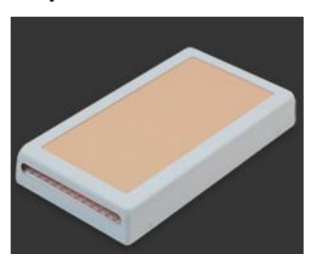

b)

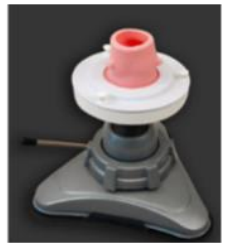

e)

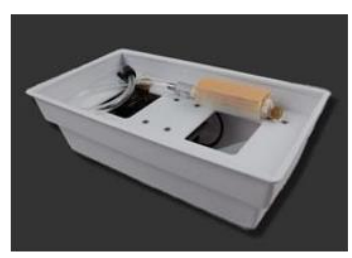

c)

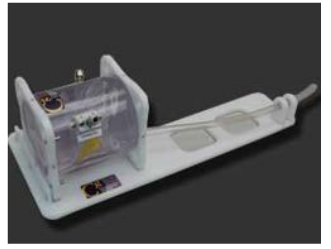

f)

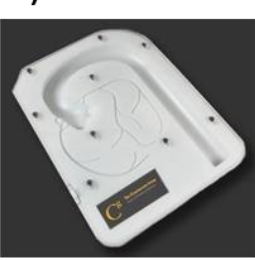

Figure 11. a) Aorta and vessel models for suture training, b) an aortic root model for valve implantation training, c) a transseptal trainer, d) a catheter insertion trainer, e) a femoral closure wet trainer and f) a cardiac array trainer (The Chamberlain Group, 2014b).

An off-pump mitral valve replacement trainer consisting of PVA left atrium connected to PVA mitral valve annulus, a left ventricle and a mock circulatory loop were developed (Figure 12) (McLeod et al., 2011). The simulator's configuration was evaluated by the use of ultrasounds and pressure measurements, which confirmed its capability of mimicking a beating heart.
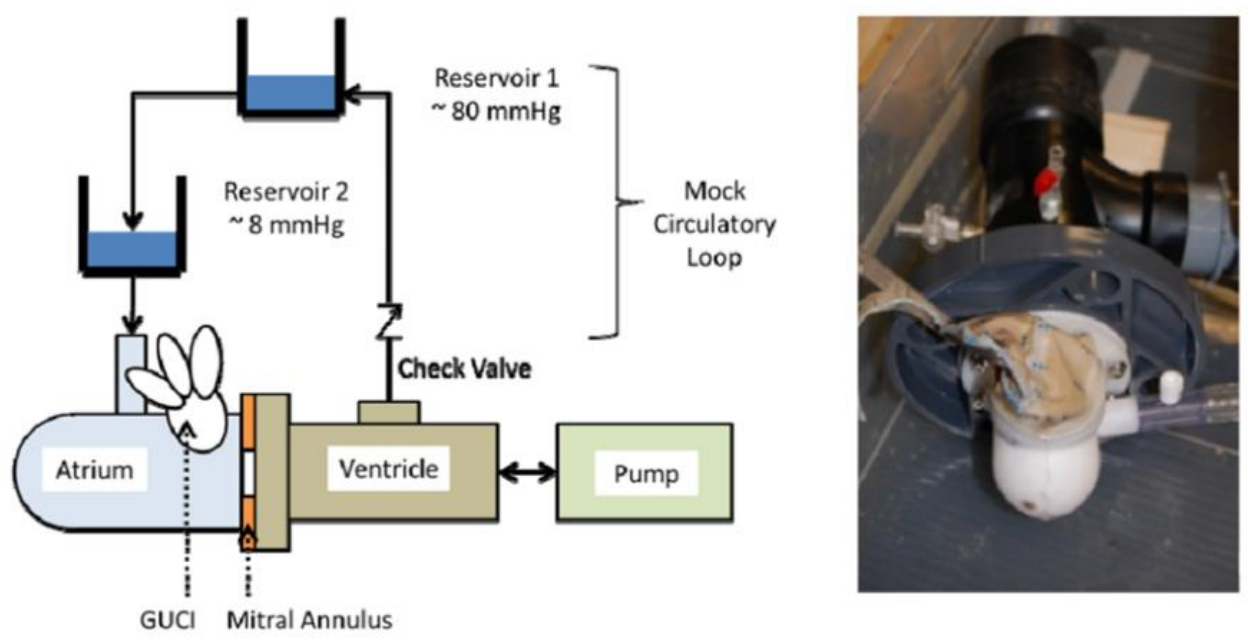

Figure 12. Mitral valve annulus model for mitral valve replacement training (McLeod et al., 2011).

\section{"Human performance simulator"}

The Ramphal cardiac simulator is a tissue-based simulator with a porcine heart connected to an air-pump system for surgical training purposes and to demonstrate the technology used during the procedures 
(Ramphal et al., 2005) (Figure 13). The haemodynamic monitoring, coronary blood flow and the beating heart are simulated in a realistic environment. Moreover, a suture-able, moving and anatomically correct heart is provided by The Chamberlain Group (Figure 13) for a better understanding of the organ in motion (The Chamberlain Group, 2014a).

a)

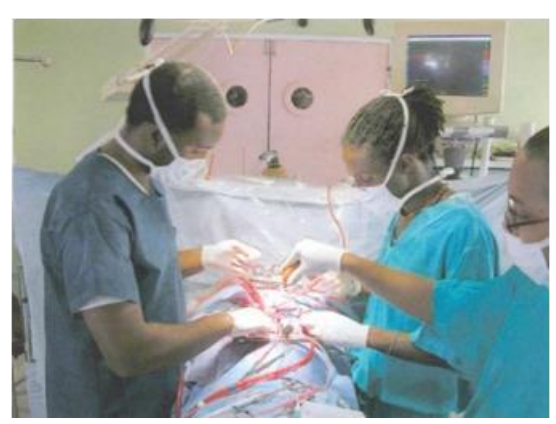

b)

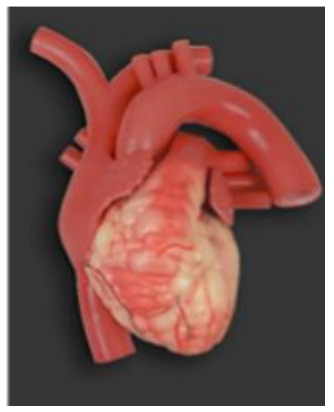

Figure 13. a) Tissue based simulator with porcine heart for complete surgery training (Ramphal et al., 2005) and b) beating and suture-able heart model (The Chamberlain Group, 2014a).

\subsubsection{Limitations of the current simulators}

Cardiac simulators are limited in their available geometries often using straight tubes to represent blood vessels. Moreover, they usually use non-realistic (mostly rigid) materials for surgical training. To our knowledge, only a few materials have been mechanically tested for comparison to the human thoracic aortic tissues (normal and aneurysmal).

Porcine aorta is also an option used in the simulation centers to compensate the lack of simulators or human donors. However, Martin, Pham, and Sun (2011) have shown the lack of representativity of the animal tissue as it would be significantly softer than aged human aortas $(p<0.001)$. The difference could be explained by a difference of composition since a histological analysis showed that porcine tissue had more elastin and less collagen than human aortas.

PVA has shown ability to simulate soft tissues but requires thermal cycling to achieve suitable crosslinking (Pazos, Mongrain, \& Tardif, 2009). PVA and silicone are both hyperelastic isotropic materials commonly used to simulate human soft tissues. Importantly, none of these materials adequately simulate the human aorta according to the medical professionals. Furthermore, the trainee should be confronted to various geometries and pathologies of aortas for optimal surgical training. Therefore, there is a need to produce anatomically correct vascular simulator with patient specific pathologies made of synthetic materials 
which mimic the biomechanics of in vivo tissues (healthy and diseased) to improve training and understanding of the human anatomy, such as the aorta.

Finally, 3D printing is a good alternative as it can make 3D objects in almost any shape with a large panel of materials (soft or hard). Therefore, the technology would be well suited for the creation of aortic simulators with various tissue characteristics and patient-specific geometries. 


\section{Chapter 4: Paper 1}

\subsection{Preface}

The following manuscript was published in 2017 in the British Medical Journal Simulation and Technology Enhanced Learning: Garcia, J., Yang, Z., Mongrain, R., Leask, R. L., \& Lachapelle, K. (2017). 3D printing materials and their use in medical education: a review of current technology and trends for the future. BMJ Simulation and Technology Enhanced Learning. doi:10.1136/bmjstel-2017-000234.

Many groups in medical education are using 3D printed models for teaching anatomy (normal and pathologies) and practicing basic manipulations, therefore we believed that a review of the creating process was an important starting point for medical educators. In the manuscript, we explained the multidisciplinary concept to the reader and developed a method to fabricate simulators guided by educational objectives and based on the case of an anatomically correct ATA (Objective 2). The main goal of this review paper was to establish an easy-to-follow and straightforward technique to create 3D printed models with anatomic and/or tissue fidelity.

This review outlined five technical steps required to complete a printed model based on the example of an ATA. Starting from the needs, the user has to 1) select the anatomical area of interest, 2) create the 3D geometry, 3) optimize the file for the printing as well as choose the 4) appropriate 3D printer and 5) materials. The fabrication of static rigid aortic phantoms from medical images (Objective 2.1.) was presented. 


\section{D printing materials and their use in medical education. A review of current technology and trends for the future.}

Justine Garcia ${ }^{1,2}$, ZhiLin Yang ${ }^{1}$, Rosaire Mongrain ${ }^{1,2}$, Richard L. Leask ${ }^{2,3}$, Kevin Lachapelle $^{4}$ *

${ }^{(1)}$ Mechanical Engineering, McGill University, Montreal, Quebec, Canada

(2) Montreal Heart Institute, Research Centre, Montreal, Quebec, Canada

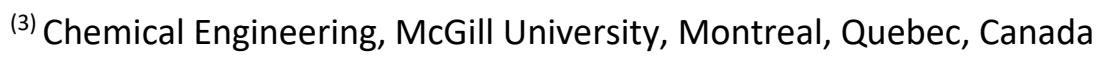

${ }^{(4)}$ Cardiovascular Surgery, McGill University Health Network, Montreal, Quebec, Canada

* corresponding author

Keywords: 3D printing, Medical simulation, Simulators, Tissue fidelity, Surgical training

\subsection{Abstract}

3D printing is a new technology in constant evolution. It has rapidly expanded and is now being used in health education. Patient-specific models with anatomical fidelity created from imaging dataset have the potential to significantly improve the knowledge and skills of a new generation of surgeons. This review outlines five technical steps required to complete a printed model: They include (1) selecting the anatomical area of interest, (2) the creation of the 3D geometry, (3) the optimisation of the file for the printing and the appropriate selection of (4) the 3D printer and (5) materials. All of these steps require time, expertise and money. A thorough understanding of educational needs is therefore essential in order to optimise educational value. At present, most of the available printing materials are rigid and therefore not optimum for flexibility and elasticity unlike biological tissue. We believe that the manipulation and tuning of material properties through the creation of composites and/or blending materials will eventually allow for the creation of patient-specific models which have both anatomical and tissue fidelity.

\subsection{Introduction}

The rapid development of 3D printing has created a new learning and teaching tool for medical education. The ability to produce patient specific in silico models from DICOM (digital imaging and communication in medicine) data derived during computed tomography (CT), magnetic resonance imaging (MRI), or 
ultrasound scanning has been coupled with new, less expensive 3D printing technology. Depending on the area of interest, these printed models demonstrate anatomical and structural fidelity consistent with the patient's actual disease process (Negi, Dhiman, \& Kumar Sharma, 2014; Rengier et al., 2010). This fidelity has allowed learners to view and understand gross pathology and structural relationships prior to surgical intervention. An improved understanding and visualization has in turn allowed surgical teams to plan interventions more accurately and guides with precision the margins of resection, modelling appropriate implant dimensions, and sometimes creating the implant itself (Malik et al., 2015; Rengier et al., 2010).

However, the vast majority of printed models are made with hard materials and only a few present some flexibility and elasticity. Although hard materials are sufficient to recreate anatomical fidelity, it has been challenging to recreate models with tissue characteristics similar to the human pathologic specimen. Patient specific practice prior to an intervention could be improved with more representative materials. This will only be possible with a firm understanding of the tissue characteristics required of the model and the capacity of the printer to blend composite materials to mimic human tissue. The type of print material which can be printed is dependent on the type of printer used. As many groups are using 3D printing technology and many more wishes to enter into this expanding field, we feel that a review of the 3D printing process would be an important starting point for medical educators.

As with every educational tool, the appropriate construction and use of these models is guided by educational objectives. Once the need has been established, there are essentially five important and often related steps to the 3D printing process to create patient specific models which have anatomic and/or tissue fidelity: 1) Capture the area of interest, 2) Creation of 3D geometry from dataset specific to the area of interest, 3) Transformation of the 3D object to a file ready for printing, 4) Selection of the appropriate 3D printer, 5) Selection of an appropriate use of materials (Figure 14). The main focus of the review is to describe the process required for the creation of 3D patient specific models and by way of example, we highlight the stepwise 3D printing process by referring to the creation of a thoracic aorta with a root aneurysm throughout the review. We also discuss the available 3D printed materials best suited for various tissue types. Finally, we suggest future directions and areas of research to advance the field of printed materials. 

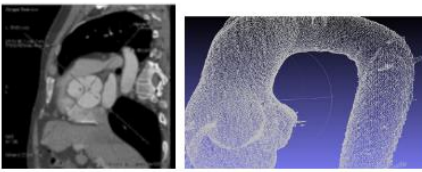

Capture the

area of

interest from

the medical

imaging
Creation of

3D geometry

from dataset

specific to the

area of

interest

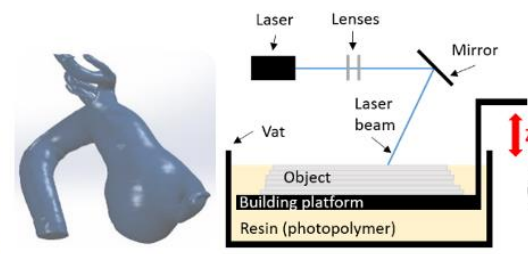

Transformation

of the 3D object

to a file ready for printing appropriate 30

printer
Selection of the
Selection of

the

appropriate

use of

material

Figure 14. Steps required in the creation of a 3D printed model in health care education. Reprinted with permission (Garcia, Yang, Mongrain, Leask, \& Lachapelle, 2017).

\subsection{Capture the area of interest}

\subsubsection{Understanding the need}

The first step in creating a new tool for medical education using 3D printing consists in defining the educational objective. Is the need to teach anatomy, pre-procedural planning or technical skills? In addition, which anatomical area needs to be represented and how much needs to be included? Understanding the educational need or learning gap you are trying to address is crucial in creating the most educational appropriate and cost-effective model.

In planning the 3D printed model, four essential characteristics of the model should be addressed and aligned with the educational need:

1) Size: How much of the organ or anatomical area is necessary? For example, is the whole ascending aorta required to instruct learners if the purpose is to teach the anatomy of the aortic root?

2) Surrounding structures: Are the surrounding structures necessary to describe the relationships of your specific anatomical area of interest? For tumors and invasive cancer, having multiple different structures is crucial to understand relationships to tumors and plan for resection.

3) Surgical manipulation: Do you wish learners to not only view the pathologic anatomy but also perform dissection or resection? In this case, more emphasis will be placed on precise anatomic details including surrounding structures, especially if dealing with potential resection of tumors. The material characteristics of the model will be essential if you require a model which will allow one to cut, resect, and suture. 
4) Accuracy and resolution of the model: How much granular detail and resolution is required for your teaching and learning? Some models print to a resolution of $1 \mathrm{~mm}$, is this necessary?

These four considerations will have impact on the type of imaging used to capture the area of interest, the resolution required, the nature of materials and composites employed, and the type of 3D printer to be used. All of these will have a direct impact on eventual costs for the production of a 3D printed model.

In regard to the thoracic aorta, we wanted to create a 3D printed and anatomically correct model with a root aneurysm able to teach exclusively the geometry of the artery to the residents in cardiac surgery. We limited the representation of the aorta to the root, the ascending aorta, the arch and the commencing of the descending aorta (Figure 15). It also included the three major branches (from proximal to distal: the brachiocephalic artery, left common carotid artery and left subclavian artery) and the commencing of the two coronaries. We kept the real size of the artery but omitted the calcifications of the patient. High accuracy and resolution were required for the model to ensure the representation of a lot of details in the final product.

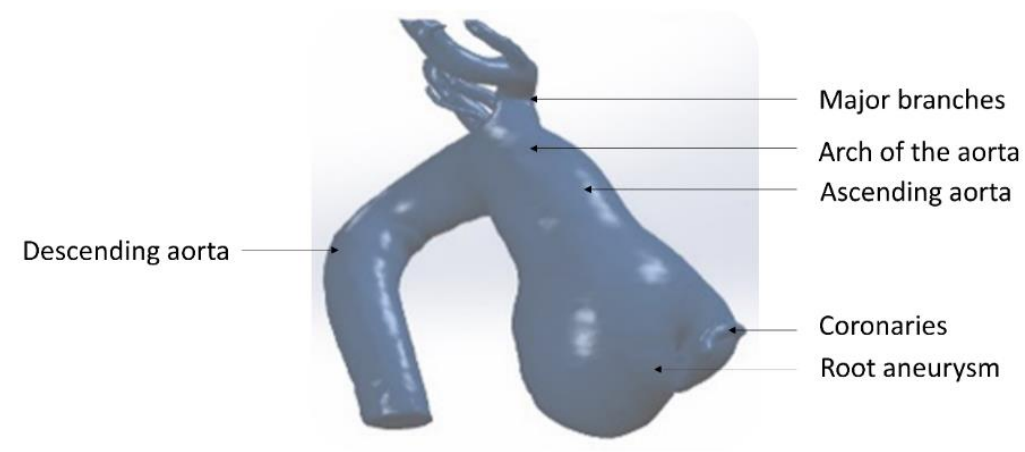

Figure 15. Steps required in the creation of a 3D printed model in health care education. Reprinted with permission (Garcia et al., 2017).

\subsubsection{Selection of the anatomy}

Once the need has been defined, discussion of these requirements should be shared with the radiologist whose expertise is to choose the adequate medical imaging process for your specific 3D printed simulator, as well as the precision of the imaging data for an appropriate representation. The concept of the medical imaging process can be seen as a multitude of $2 \mathrm{D}$ pictures taken one after the other and which are separated with a controlled thickness determined in advance, thus the 3D representation is made by simply stacking successive layer of 2D images into a 3D volume. This explains why the accuracy of the 3D 
geometry diminishes as the thickness between each slice increases (see Figure 16). Low resolution will produce large spaces between the 2D images, losing potentially important anatomical detail. The distance between two slices is generally recommended to be $1 \mathrm{~mm}$ or less (Friedman, Michalski, Goodman, \& Brown, 2016) but J. S. Matsumoto et al. (2015) found that $1.5-3 \mathrm{~mm}$ is an appropriate thickness for the chest and the abdomen and $0.4-0.75 \mathrm{~mm}$ for bones and joints. In our lab, we used a slice thickness of $0.625 \mathrm{~mm}$ in our reproduction of cardiovascular (thoracic aorta with root aneurysm) and hepatic anatomy.

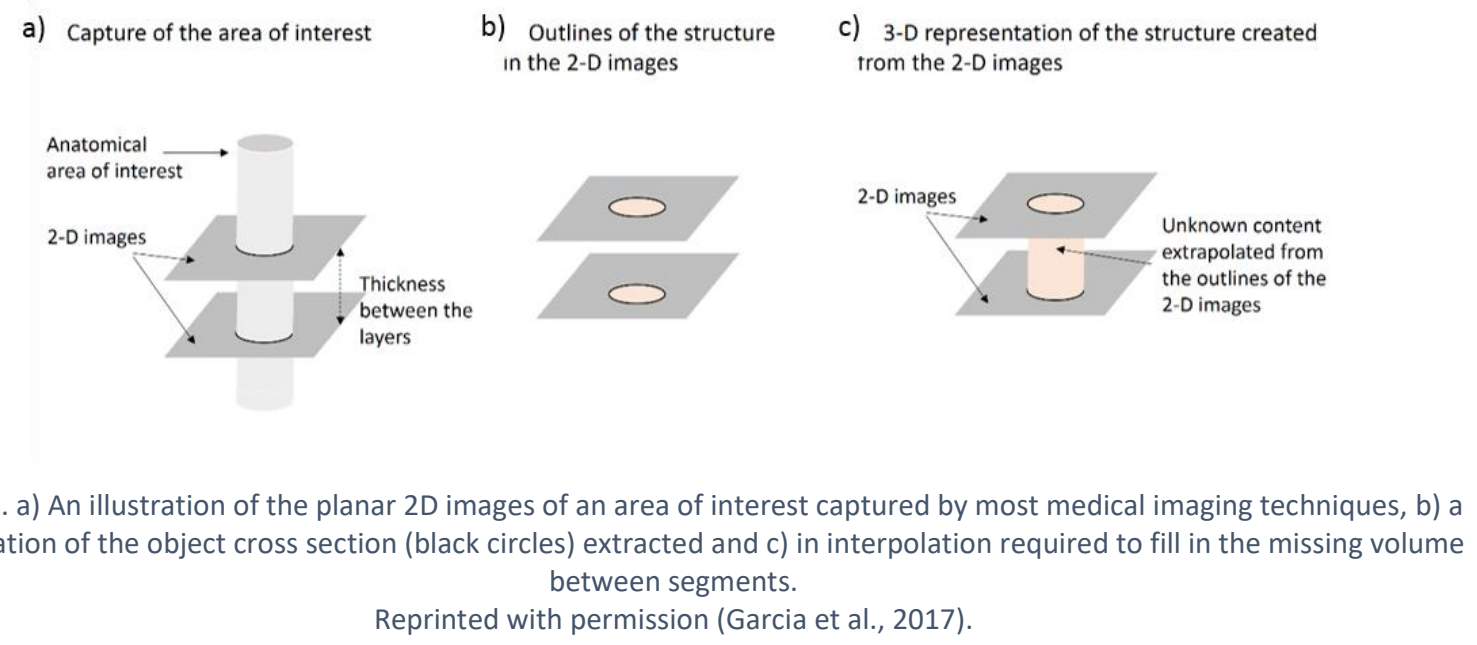

Figure 16. a) An illustration of the planar 2D images of an area of interest captured by most medical imaging techniques, b) a segmentation of the object cross section (black circles) extracted and c) in interpolation required to fill in the missing volume Reprinted with permission (Garcia et al., 2017).

Several imaging processes are used in radiology to capture 2D images of the human body but the most common technologies remain CT and MRI. For both methods, contrast agents are injected into the patients before each acquisition for a better distinction between the tissues by enhancing the contrasts of the structures of interest. Controlling the noise and resolution of the images also impacts on the quality of the 2D images (Ogden et al., 2015). A very high resolution of data imaging is always recommended, however, depending on the needs and the capabilities of the 3D printer selected for the fabrication, high resolution may not be required. As will be reviewed later, only a few printing technologies and apparatus models can reproduce very fine details.

If one is interested in printing structures of the cardio-thoracic and vascular systems the best modalities to visualize arterial and venous vessels (Friedman et al., 2016) are CT angiography and MRI angiography. $\mathrm{CT}$ is the method we chose to capture the geometry of the thoracic aortic model. CT and MRI both require electrocardiogram-gated acquisitions to identify the systolic and/or diastolic geometries while reducing body motion (Ripley et al., 2016) essential to avoid blurry images thus improving the visibility of aortic 
valves while capturing the geometry of the aorta for instance. In the literature, CT datasets have also been used for other aortic models (Schmauss et al., 2012) and geometries as hearts with congenital diseases (Shiraishi, Yamagishi, Hamaoka, Fukuzawa, \& Yagihara, 2010), aneurysm of the celiac trunk (Salloum et al., 2016), bronchial tree (Deferm, Meyns, Vlasselaers, \& Budts, 2016) and oesophagus (Dickinson et al., 2015). Similarly, structures of a pulmonary valve (Armillotta et al., 2007), cardiac tumors (Schmauss, Gerber, \& Sodian, 2013) and thoracic aortas (Markl, Schumacher, Küffer, Bley, \& Hennig, 2005) have been replicated from MRI images. Datasets from multiple imaging sources can be merged for optimal results. Indeed, pulmonary arteries for thoracic surgery training have been created with a mix of conventional CT and CT angiography images (Kurenov, lonita, Sammons, \& Demmy, 2015). Similarly, models for cardiac surgery and interventional cardiology have been developed with CT and MRI (Schmauss, Haeberle, Hagl, \& Sodian, 2015) and mitral valve models by CT and transesophageal echochardiographic (Vukicevic, Puperi, Grande-Allen, \& Little, 2016).

Models that replicate soft tissue and the skeleton, such as the assembly of lungs and a thorax are made from CT images (Gillaspie et al., 2016), and require optimization of imaging for multiple tissue densities. Friedman et al. (2016) have used CT with a standard algorithm to capture bone geometries minimizing artifacts along the contacts between bone and soft tissues, while using MRI to provide high contrast between the cortical bone and the surrounding tissues. Temporal bone 3D reconstructions have been created from CT (Cohen \& Reyes, 2015; Hochman, Kraut, Kazmerik, \& Unger, 2014; Suzuki et al., 2004) or microCT (Hochman et al., 2015), while cone beam CT (cone formed by X-rays) and multislice CT (higher number of slices, thus a better resolution) are known to be other appropriate methods in craniomaxillofacial surgery (Huotilainen et al., 2014).

In the field of neurosurgery, CT and MRI have been used to reproduce vascular and brain tissue (Kondo et al., 2016; Ploch, Mansi, Jayamohan, \& Kuhl, 2016). CT imaging data has been used in otolaryngology-head and neck surgical training for the creation of endonasal, paranasal sinuses, skull base and mandibular phantoms (Chan et al., 2015), a malformed skull (Levi et al., 2002), as well as a cortical brain tumor structure through the skin, bone, dura mater and surrounded by normal brain (Waran, Narayanan, Karuppiah, Owen, \& Aziz, 2014). Nevertheless, single CT datasets can also provide enough information to proceed a geometry extraction from the 2D images, as in the case of phantoms for endovascular aneurysm repair in complex neck anatomy (Tam, Laycock, Brown, \& Jakeways, 2013) and a cerebral aneurysm (Wurm, Lehner, Tomancok, Kleiser, \& Nussbaumer, 2011). 
Finally, when metallic elements are involved in CT imaging (prosthesis, plate, screw, etc.) the artifacts distorting the geometry of the structure caused by beam-hardening or scatter can be well controlled with a dual energy CT giving a second less powerful X-ray after the conventional one for a better image quality (Friedman et al., 2016).

\subsection{Creation of 3D geometry from dataset specific to the area of interest}

Once an adequate clinical imaging dataset has been acquired, an in silico geometric object needs to be created. As a rule, one 3D object means one component that will be fabricated from one specific material. For instance, if the assembly is composed of two distinct objects such as a bone and ligament, both geometries need to be clearly defined separately before joining them back together as an assembly before the printing for a proper fabrication (see Figure 17). Often the two objects will be made of different materials or printed in different colours for clarity.

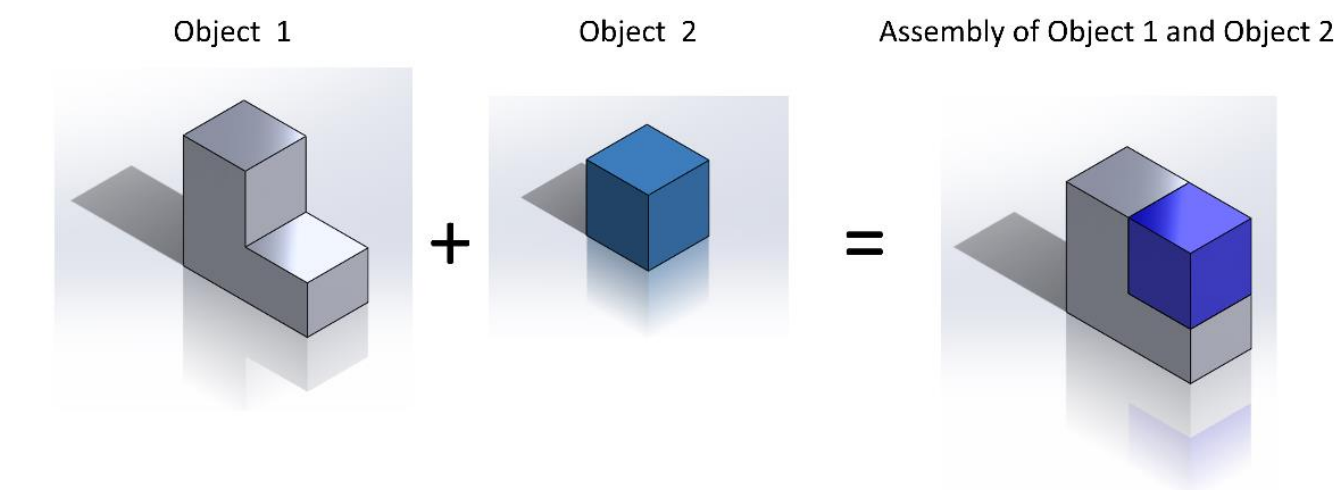

Figure 17. Two distinct objects made of different materials or printed in different colors creating an assembly. Reprinted with permission (Garcia et al., 2017).

The thoracic aortic model with root aneurysm did not include any secondary elements as the calcifications of the patient for instance. Therefore, a single 3D printed material was required to uniformly recreate the walls of the artery.

When different objects are involved, it is best to define them directly from the acquired DICOM image by extracting points along the outline of the anatomical structure to define its geometry. This extracting process is called a segmentation which is often threshold-based (Huotilainen et al., 2014) therefore it is using the colour contrast between tissue densities to separate tissue structures into different objects 
(Ripley et al., 2016). Some may prefer defining the geometries afterwards by dividing a bigger structure, however, this can be a complicated task for non-experienced design software users.

When defining objects from DICOM images, the contrast levels or thresholds of the segmentation are highly dependent on the image. Threshold levels often vary between, patient images and image quality from one slice to another may require altering threshold levels. Therefore, verification that the anatomical structures of interest are properly identified from the imaging data needs to be checked. Once threshold levels have been defined, segmented points can be then extracted from the dataset along the outline(s) of the structure on the 2D images by appropriate segmentation software (Figure 18). Each 2D image (or slice) of the 3D imaging dataset are analyzed individually and data points are created along the perimeter (or outline) of the structure. The number of data points describing the geometry of the structure for one slice depends on the size of the structure at that specific cross-sectional view. Number of points should increase with the size of the perimeter of the structure to avoid losing details of the geometry. The data points from all slices are collectively called a point cloud as seen in Figure 18 representing the aortic model with root aneurysm. We were only able to select the inner lumen of the geometry due to the lack of visibility of the thickness of the walls. The thickness would have to be determined later after the creation of the 3D geometry of the inner lumen of the aorta.

When stacking successive slices of 2D images to create an object, such as an aorta, each point is represented in the larger point cloud of the aorta. The multiple points of the cloud will be used to create the surface of the object.
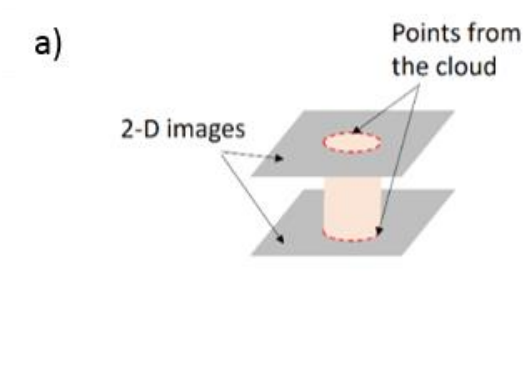

b)

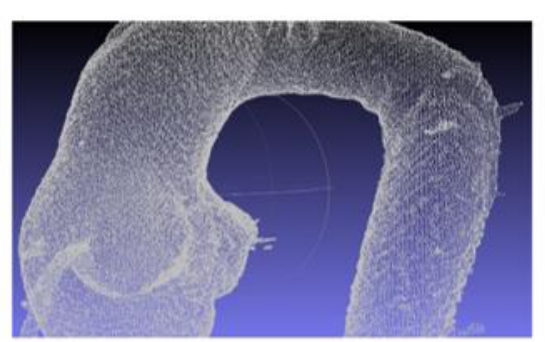

Figure 18. a) A point cloud created from two 2D images and an example of b) a point cloud obtained from the inner lumen of an ascending aorta of a patient from the Royal Victoria Hospital (Montreal, Canada) with a $0.625 \mathrm{~mm}$ thickness between the slices. Reprinted with permission (Garcia et al., 2017).

Segmentation is necessary as it extracts the points used to create a contour of the structure which will be read and printed by a 3D printer in successive layers. Segmentation can be performed manually or 
automatically. Manual segmentation is extremely slow but suitable for almost any anatomical structures even with complex geometries and in presence of artifacts, as the human visual system is still far superior to any algorithms in term of pattern recognition (Von Ahn, Maurer, McMillen, Abraham, \& Blum, 2008). However, it requires an experienced user with a good 3D appreciation to diminish the risks of potential errors since low contrasts or overlapping tissues may complicate the task (Huotilainen et al., 2014). Automatic segmentation is much quicker, but can only be used for easily identifiable or high contrast structures (Friedman et al., 2016). Regardless, in any realistic setting, segmentation is always done via a mix of manual and automatic segmentations by leveraging the advantage of both techniques to produce the best results as we did for the thoracic aorta after we verified the dataset for proper gating before any attempt at segmentation. The segmentation procedure of a very clear dataset can be done within a few hours, while a very unclear dataset may take up to a week. Only a few slices were segmented at once to optimize between time and quality. Each time a set of images has been segmented, the segmented slices were checked one by one to ensure that the lumen in each slice was given a proper boundary; if not, manual correction was made. 3D visualization of the segmented section was generated to ensure the quality of segmentation. Special care was given to the aortic root where the shapes of the valves were extrapolated from the images of the blood flow, however we knew that they were closed as the dataset used were all taken during diastole.

\subsection{Transformation of the 3D object to a file ready for printing}

The point clouds defining the objects are exported into a mesh processing software to remove all visible artifacts manually created by selecting unnecessary points. Deformations can create cavities or peaks deforming the original geometry of the structure (Figure 19). Once the in silico model is optimized, a computational mesh (a surface composed of flat polygonal elements to approximate the geometry) is then created by connecting the points from the cloud as a grid in order to describe the object. 
a)

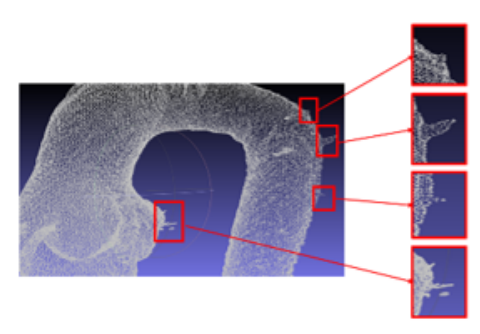

b)

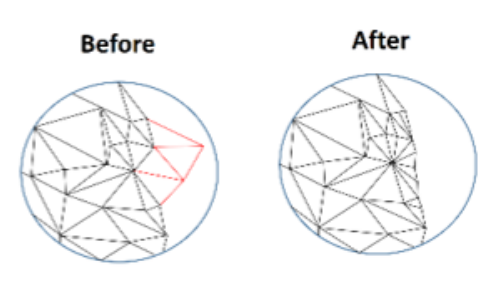

Figure 19. a) Artifacts from the point cloud of an ascending aorta of a patient from the Royal Victoria Hospital (Montreal, Canada) and b) an artifact before and after the mesh smoothing. Reprinted with permission (Garcia et al., 2017).

At this stage of the process, the interconnected elements of the model may present new artifacts that should be removed, as well as surface irregularities or sharp edges. Most irregularities can be fixed by simply smoothing the surfaces with algorithms meant to remove details from the object (Figure 19). The procedure took less than a half hour for the case of the aorta. However, the user should be aware that excessive smoothing can also deteriorate the resolution of the 3D model. Too little smoothing generally increases the number of elements required to define the object while too much smoothing has the opposite effect. It is recommended to control the number of elements since file size increases with the number of elements, thus making computer manipulations more difficult. In our lab, we use the opensource meshing software (MeshLab, Italy).

For the thoracic aortic model with root aneurysm, once the point cloud of the inner lumen of the artery was cleaned we removed the vasa vasorum (small vessels) and other irregularities from the geometry. The mesh was then repaired and the number of elements decreased to simplify the geometry and make it printable.

Manipulations of meshed anatomical geometries require powerful computers with good processors (Intel i7 with $3.2 \mathrm{GHz}$ or better), memory ( $8 \mathrm{~GB}$ or better) and a good graphics card (with dedicated memory). Gaming computers and engineering workstations generally has the capacity to handle most 3D models, however with particular large files, custom made workstation may be required. An optimization of the model with a proper degree of smoothness and a reduction of the element numbers describing the shape of the structure, can diminish the processing time for visualization and editing.

Sometimes, images obtained from the radiology may have poor resolution with no distinct boundaries, thus sections of the structure may not be fully defined. This lack of information can be replaced with a 
manual reconstruction by computer aided design, 3D graphics software and even some mesh processing programs that can make very basic modifications of the geometry. For example, we have previously used simple spherical segments to replace and approximate aortic valve leaflets missing from CT images, see Figure 20.
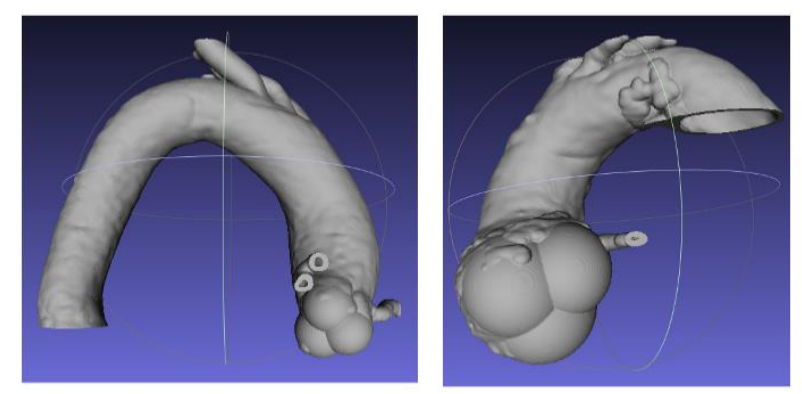

Figure 20. Spheres created to approximate the shape of a patient-specific aortic root, open source Meshlab (MeshLab, Italy). Reprinted with permission (Garcia et al., 2017).

Such software is also useful to make modification of objects if the printed model is made of several materials. As mentioned in the previous section, the geometry of each object of the model has to be defined individually, hence the easiest model would be made of only one object/material. To define the shape of the objects, it is recommended to use Boolean operations which are mathematical operations for volumes such as the addition (also called union) and subtraction, both most frequently used in medical simulation (Figure 21). For instance, if the user wants to print a liver containing a vein, the former will be removed from the liver with a subtraction giving two distinct objects (one for the vein and one for the hollowed liver without the vein) which will be finally printed together as an assembly. This step is crucial to avoid any volume overlapping (Figure 21 ) that would produce printing errors. Similarly, if a ball-shaped object made of the same material as the rest of the structure needs to be added to the geometry to simulate an aneurysm or a tumor, a union operation is required to create a unique object. 
a)

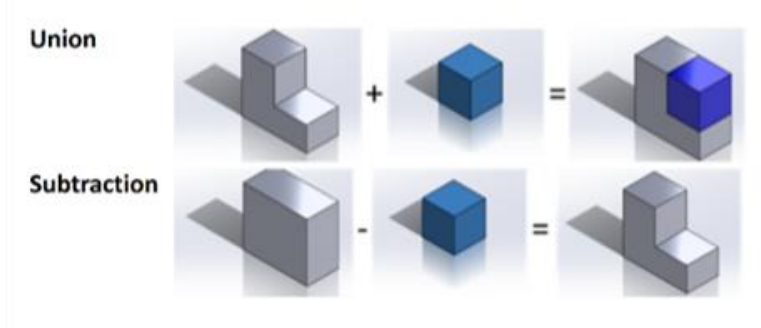

b)

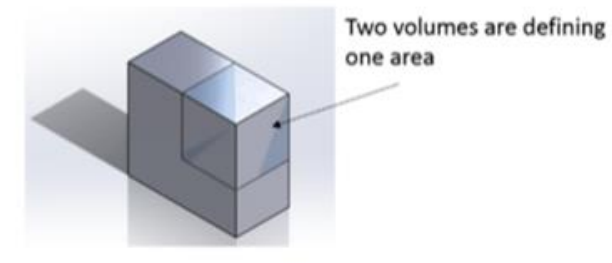

Figure 21. a) Most frequent Boolean operations to create objects (union and subtraction of volumes) and b) volume overlapping that should be avoided in any circumstances.

Reprinted with permission (Garcia et al., 2017).

To finalize the model, repair algorithms can highlight and correct all potential errors in your meshes which are not easy to see by eye, such as holes from non-connecting triangles or bad edges, overlapping triangles and small triangles that you may have missed and which are not part of the main geometry for instance (Figure 22). The software netfabb (Autodesk, California, USA) is open access and can perform these tasks for very simple geometries. For more complicated models, such as the patient specific aorta of Figure 19, a professional paid version is required.

To create hollow objects, a thickness can be added to the mesh surface otherwise the whole model will be automatically filled and made of solid unless it is clearly specified in the geometry. This is the method we used to create an aortic wall of $2 \mathrm{~mm}$ from the 3D object of the inner lumen in order to create a 3D printed model with the geometry of the in vivo artery.

a)

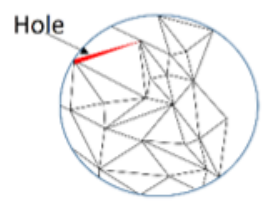

b)

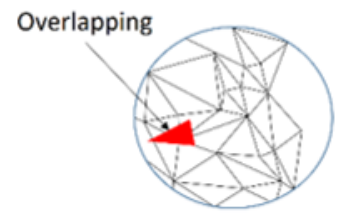

c)

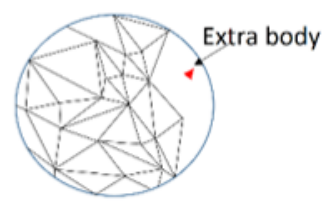

Figure 22. Mesh errors of a) non-connecting triangle, b) overlapping triangles and with c) an extra body that is not part of the main geometry.

Reprinted with permission (Garcia et al., 2017).

The aforementioned steps are important steps but also probably the most difficult in the creation of 3D printed models. Once again, if your mesh contains error(s), the printer might not be able to fabricate the object at all or mistakes would occur during the printing. Sometimes, it may be easier to remove and 
replace an entire surface instead of trying to fix the original object. Knowledge in 3D computer-aided design is a significant asset.

Once the 3D in silico solid model is finalized, the file can be then exported to the STL (stereolithography) standardized format for 3D printing before being uploaded to the 3D printer for fabrication. These files contain information related to the generated mesh, as well as orientation and position of the structure. Once again, only one object/material/colour can be assigned to one file. If several objects/materials/colours are required for the final 3D model, each object should be saved as a different file (see Figure 23) and all have to be uploaded simultaneously to the printer as an assembly. Material/colour is then attributed to each object with the 3D printer software.

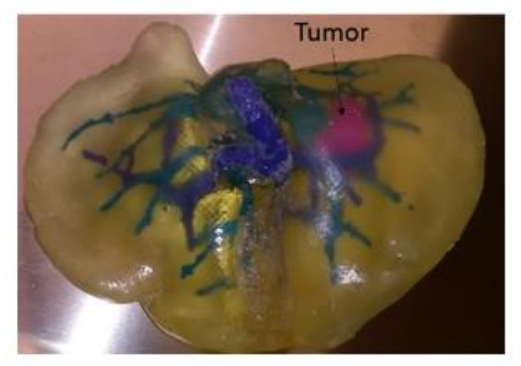

Figure 23. Multicolor 3D printed liver with a tumor (pink) of a patient from the Royal Victoria Hospital (Montreal, Canada) for surgical planning of a diseased liver. The print contain the portal vein, the hepatic vein as well as the tumor.

Reprinted with permission (Garcia et al., 2017).

\subsection{Selection of the appropriate 3D printer}

Selecting the appropriate 3D printer to fabricate a patient specific model can be a challenge. It is most helpful to have clarified the educational needs and the intended use of the model prior to the printing process. This will help guide the selection and requirement for the 3D printer. Herein we describe the most suitable methods for printing which are currently available and will discuss the pros and cons for each of them.

\subsubsection{Description of the 3D printing methods}

The general concept of 3D printing is the fabrication of objects as a succession of layers (see Figure 24). Each layer of an object (or an assembly of objects) has the same thickness and the thickness depends on the accuracy of the method and the machine chosen. Moreover, 3D printers are not necessarily limited 
to one material, for instance material (A) can be used for one object composed of layers 1, 2 and 3, while a second object (layer 4) can be fabricated with the material (B). This information has to have been previously defined by the geometrical files converted in STL that you have created for each distinct object. Some machines are even able to mix materials for one (or more) layer(s) in order to obtain specific colors or material properties.

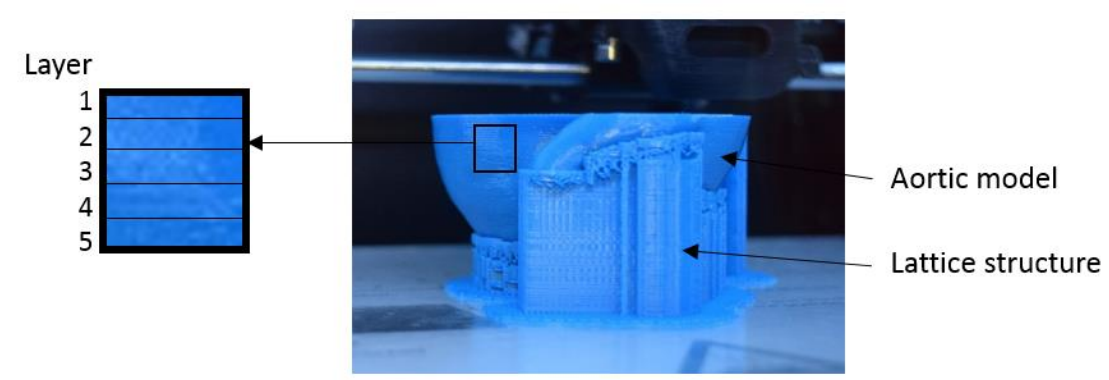

Figure 24. Aortic model in process for being printed on a FDM 3D printer by successive layers of ABS plastic ( $0.3 \mathrm{~mm}$ thickness). Reprinted with permission (Garcia et al., 2017).

Materials used in 3D printing are transformed during the fabrication of a model by changing their consistency. This process, commonly called the cure, can take the form of 1) a melting of hard filaments to give the desired form of the model by material distortion, 2) a liquid solidification for the construction of a solid structure, and 3) a powder solidification.

1) Melting of hard filaments: Filaments are melted in FDM (fused deposition modeling) then injected through a nozzle to a bed while cooling and solidifying during extrusion (Figure 25). These printers are inexpensive and fast but are limited to rigid material. FDM needs a scaffold or bed to support the object during the printing.

2) Liquid solidification: Liquid solidification is used in SLA (stereolithography) and PJ (polyjet) technology. SLA is the oldest method which contains liquid in a vat solidified with an ultraviolet (UV) laser controlled by lenses and mirror reflection, while a building platform is moved down for a layer-by-layer fabrication (Figure 25). A lattice structure is created to support the object. In contrast, PJ uses jets of liquid to build up thin layers of liquid before UV curing by sliding the head in the $x$ and $y$ axes then a moving platform for the $\mathrm{z}$ axis to build up successive layers (Figure 25). PJ is suitable for a wide range of coloured materials with specific properties (rubber-like material) (Biglino et al., 2013; Cloonan et al., 2014; Oxman, Tsai, \& Firstenberg, 2012; Reiter \& Major, 2011). 
3) Powder solidification: Finally, powder materials can be solidified by SLS (selective laser sintering) and BJ (binder jetting). SLS uses a laser to create the surface of an object by sintering a powder. When a layer is done, the build plate is stepping down and covered with a new layer of powder using rollers to keep a constant thickness (Figure 25). At the end of the process, all powder is removed with compressed air and recycled (Wong \& Hernandez, 2012). A wide range of materials are provided for SLS and no scaffold or support material is required since the unsintered powder provides support of the object during the 3D printing. Similar to the SLS, BJ has a platform covered with powder which is moved down while being solidified with a selective spraying of liquid binder, thus no support material is needed for the fabrication of the object (Figure 25). This technology offers a wide range of materials as far as a powder can be combined with a liquid with enough viscosity to form droplets (Guo \& Leu, 2013). In addition, mechanical properties of the structures obtained by $\mathrm{BJ}$ in medical simulation are generally tuned by post-processing techniques, such as drying and/or heating. Both have an impact on the mechanical properties of the materials (hardness) however none can be precisely replicated for each fabrication resulting in variations or the characteristics from a model to another (Hochman et al., 2014). 

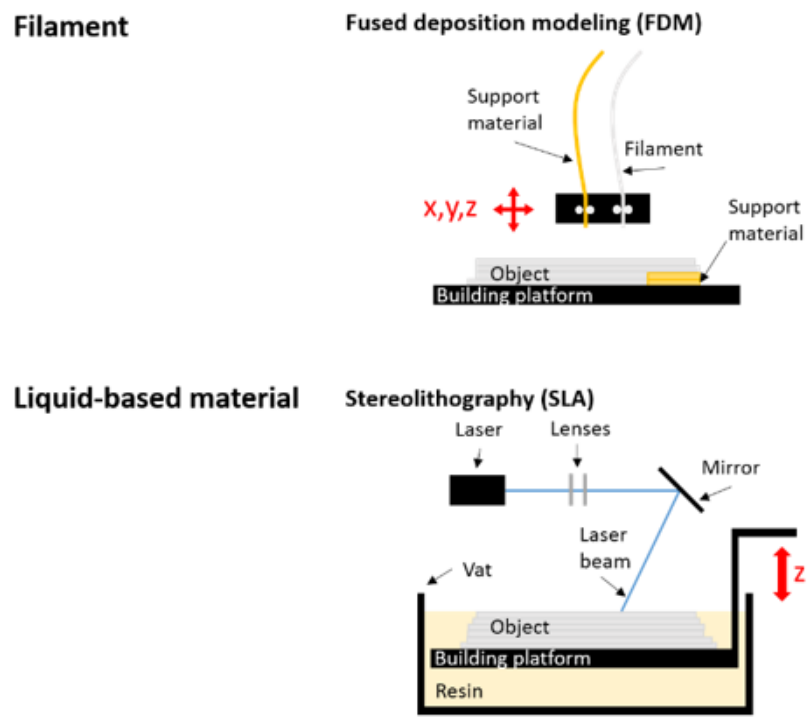

Polyjet (PJ)

Powder-based material Selective laser sintering (SLS)

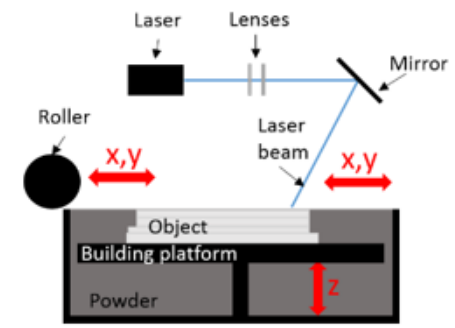

Binder jetting (BJ)

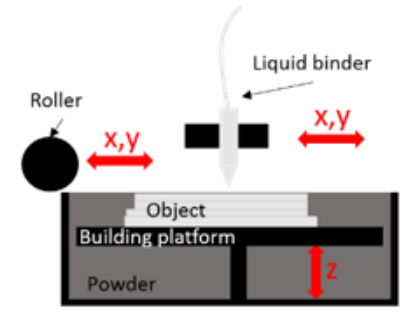

Figure 25. Rapid prototyping methods with red arrows indicating the directions of motion ( $x, y, z$ axes). Reprinted with permission (Garcia et al., 2017).

\subsubsection{Support material}

Material cannot be deposited on empty space thus models with overhangs, as Figure 26, often require filler or support material in lattice (or scaffold) forms. Moreover, the filling material is meant to strengthen the structure during the printing, thus avoiding distortion of the model while the material is being cured.

With filaments (FDM) or liquids (SLA), support materials provide lattices which can be easily removable by hand with a cutting tool, however they often leave undesired impressions on the surface requiring an additional polishing for a good finish. This step of the process is extremely delicate due to the risk of damaging the model by losing details of the geometry. A few support materials can also be easily removed when the model is complete by dissolution, as the PVA in FDM soluble in water. 
In PJ, volumes of cured waxy support material are used to fill the overhangs. For the soluble ones, sculpting tools are generally used to clean the models, as well as water jets. Otherwise, bath of solutions can remove on its own the filling necessary for the printing.

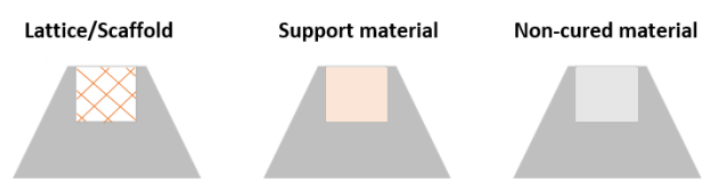

Figure 26. Structure with an overhang filled with a lattice structure, support material or non-cured material. Reprinted with permission (Garcia et al., 2017).

If your 3D printer is fabricating models from powders (BJ, SLS), the overhangs will be filled with uncured material playing the role as support. This is also very easy to clean up.

It is important to note that models printed with insoluble lattices or waxy support material can be extremely complicated to clean up especially when hollows are not easily accessible by hand or with tools.

\subsubsection{Costs of the printing: 3D printer, material and technical support}

The easiest, most accessible and cost-effective technology is the FDM (Wong \& Hernandez, 2012), which is also the least expensive technology, see Table 2. Price generally increases from BJ, to SLA and finally the SLS and PJ.

Regarding the material costs, FDM filaments are once again the least expensive material of all 3D printer type and should be considered for large print volume where material property and high print resolution are not the main concern. Moreover, they are easy to store and use (Armillotta et al., 2007) leading up to the most user-friendly process. SLA and PJ can provide high resolution but are also much more expensive. SLA has the highest resolution of all types of 3D printer, while PJ printers allow the use and mixing of more than one material in a single print. BJ is also cost-effective and the overall cost of the printing low. The powders are somewhat more expensive than the filaments for FDM, nevertheless they have the advantage of being reusable when uncured but used as support for previous printings. Therefore, there is very little waste of material in comparison to liquid and filament methods. Moreover, BJ printers are fast, easy to use and maintain. Main consumable components are inexpensive and easily replaceable. BJ models are, however, more fragile than FDM prints when untreated. 
In terms of technical requirements, risks of malfunction of the printer in FDM are rare and easy to solve. Common issues can be summarized as a melting or sliding problem of the material through the nozzles provoking a clogging, or when part does not attach properly on an inadequately heated print bed. Most failures in FDM printer trend to be caused by the design of the 3D model rather than any issues with the machine itself. The technology requiring the most extensive technical support is the PJ. In order to avoid any clogging with a potential solidification of the liquid in the nozzles a technician should run a weekly maintenance by using all loaded materials if there is no scheduled printing to provide a continuous flow of materials in the printer. Moreover multiple pieces of the machine need to be frequently cleaned using very specific techniques, and mistakes can easily damage the equipment. Furthermore, the 3D printer should be kept in a specific environment with a ventilation system, and the materials protected from light. PJ machines also should not be turned off unless there is a long period of time (months) without printing. This involves a large waste of material by removing the liquid to empty the nozzles, thus maintenance costs become higher if you are using PJ sporadically than if you are continuously printing. Liquids for SLA also need to be kept away from light but the machines do not require weekly maintenance, such as SLS and BJ. They can be used whenever the user wants to print an object and all are easy to use. The most complicated part of the process may be the material removal after the printing. For Binder jet printers, excess material as well as the build area has to be manually vacuumed to remove unbound powder. For SLA printers, the print tank must be carefully inspected to ensure no cured material remains in the tank, for it may interfere with the curing process of future prints.

Table 2. Main characteristics of the rapid prototyping methods: Stereolithograpy (SLA), PolyJet (PJ), Fused deposition modeling (FDM), Selective laser sintering (SLS), Binder jetting (BJ). Reprinted with permission (Garcia et al., 2017).

\begin{tabular}{|c|c|c|c|c|c|c|}
\hline \multirow{2}{*}{\multicolumn{2}{|c|}{$\begin{array}{l}\text { Material based type } \\
\text { Method }\end{array}$}} & \multicolumn{2}{|l|}{ Liquid } & \multirow[b]{2}{*}{$\begin{array}{l}\text { Filament } \\
\text { Material } \\
\text { melted and } \\
\text { solidified by } \\
\text { cooling }\end{array}$} & \multicolumn{2}{|l|}{ Powder } \\
\hline & & $\begin{array}{l}\text { Liquid } \\
\text { solidification }\end{array}$ & $\begin{array}{l}\text { Liquid } \\
\text { solidification }\end{array}$ & & $\begin{array}{l}\text { Material sintered } \\
\text { by laser }\end{array}$ & $\begin{array}{l}\text { Material } \\
\text { solidified } \\
\text { with liquid } \\
\text { binder }\end{array}$ \\
\hline \multicolumn{2}{|l|}{ Process } & SLA & PJ & FDM & SLS & $\mathrm{BJ}$ \\
\hline \multicolumn{2}{|c|}{$\begin{array}{l}\text { General building speed } \\
\text { (slow/intermediate/fast) }\end{array}$} & Intermediate & Intermediate & $\begin{array}{l}\text { Slow (Wong } \\
\text { \& } \\
\text { Hernandez, } \\
\text { 2012) }\end{array}$ & $\begin{array}{l}\text { Fast (M. S. Kim, } \\
\text { Hansgen, \& Carroll, } \\
\text { 2008) }\end{array}$ & $\begin{array}{l}\text { Very fast } \\
\text { (M. S. Kim } \\
\text { et al., 2008) }\end{array}$ \\
\hline \multirow[t]{2}{*}{$\begin{array}{l}\text { Printing } \\
\text { quality }\end{array}$} & $\begin{array}{l}\text { Accuracy } \\
\text { (low/intermediate/high) }\end{array}$ & $\begin{array}{l}\text { High (M. S. } \\
\text { Kim et al., } \\
\text { 2008; } \\
\text { Rengier et al., } \\
2010 \text { ) }\end{array}$ & $\begin{array}{l}\text { High (Wong } \\
\text { \& } \\
\text { Hernandez, } \\
\text { 2012) }\end{array}$ & $\begin{array}{l}\text { Low (Rengier } \\
\text { et al., 2010; } \\
\text { Wong \& } \\
\text { Hernandez, } \\
\text { 2012) }\end{array}$ & $\begin{array}{l}\text { Intermediate } \\
\text { (Rengier et al., } \\
\text { 2010) }\end{array}$ & $\begin{array}{l}\text { Low (M. S. } \\
\text { Kim et al., } \\
2008)\end{array}$ \\
\hline & $\begin{array}{l}\text { Resolution of a typical } \\
\text { machine }\end{array}$ & 5-25 micron & $\begin{array}{l}15-30 \\
\text { micron }\end{array}$ & 100 micron & 125 micron & 100 micron \\
\hline Costs & $\begin{array}{l}\text { Machine } \\
(\$ / \$ \$ / \$ \$)\end{array}$ & $\$ \$$ & $\$ \$ \$$ & $\$$ & $\$ \$ \$$ & $\$ \$ \$$ \\
\hline
\end{tabular}




\begin{tabular}{|c|c|c|c|c|c|}
\hline $\begin{array}{l}\text { Material } \\
(\$ / \$ \$ / \$ \$)\end{array}$ & $\$ \$$ & $\$ \$ \$$ & $\$$ & $\$ \$$ & $\$ \$$ \\
\hline $\begin{array}{l}\text { Overall cost for printing an } \\
\text { object all costs included } \\
\text { (low/intermediate/high) }\end{array}$ & $\begin{array}{l}\text { Medium } \\
\text { (Rengier et } \\
\text { al., 2010) }\end{array}$ & $\begin{array}{l}\text { High (M. S. } \\
\text { Kim et al., } \\
2008 \text { ) }\end{array}$ & $\begin{array}{l}\text { Low (Rengier } \\
\text { et al., 2010; } \\
\text { Wong \& } \\
\text { Hernandez, } \\
\text { 2012) }\end{array}$ & $\begin{array}{l}\text { High (Rengier et al., } \\
\text { 2010) }\end{array}$ & $\begin{array}{l}\text { Very low } \\
\text { (M. S. Kim } \\
\text { et al., 2008) }\end{array}$ \\
\hline
\end{tabular}

\subsubsection{Building speed, accuracy and quality of the fabrication}

The liquid-based technologies (SLA, PJ) provide the best accuracy and the powder-based (SLS, BJ) printers have the fastest build speed. This is the reason why we chose PJ process to create the thoracic aorta with root aneurysm for teaching purposes to ensure a high-quality printing that can replicate details in the geometry of the artery. We wanted indeed our 3D printed model to be as accurate as possible to show the in vivo geometry of a diseased aorta to the non-experienced surgeons. Methods using a support fiber lattice may degrade surface quality and may require hand finishing to remove the undesired impressions on the surface as explained above (Wong \& Hernandez, 2012). Nevertheless good machines with a high resolution requiring support fiber scaffolds may still fabricate an object with a good surface roughness and definition (G. D. Kim \& Oh, 2008) as the FDM and SLA (Table 2).

In addition, powder fineness will impact the quality of the printing by SLS (Ventola, 2014), as well as the quality of the laser. This is the case for any printer using UV for solidification (SLA, PJ) and sintering (SLS). Powder sintering can also create pores/voids in the model (Guo \& Leu, 2013) thus causing fragile structures with a low stiffness (G. D. Kim \& Oh, 2008) in BJ, in opposite to the solid structures created by SLS (Wong \& Hernandez, 2012). In contrast, liquids tend to create more homogeneous material properties (G. D. Kim \& Oh, 2008; Wong \& Hernandez, 2012) that can be hard as well as elastic (Blanco, Fernandez, \& Noriega, 2014).

Finally, good design and positioning of the object are required for an optimal fabrication regardless the technology itself. Mechanical property variations may be observed on the model depending on the method and parameters chosen, such as an anisotropic behavior corresponding to a higher resistance to deformation of the layer direction directly related to the building direction (M. S. Kim et al., 2008). Stiffness reductions can also be seen on the side of the object in contact with the support material which will be less exposed to the UV radiation cure during a polymerization (Blanco et al., 2014). 


\subsubsection{Selecting the right printer for teaching}

Depending on the educational need listed below recommendations of methods are made.

1) Teaching anatomy, patient education: To teach the anatomy and explain the pathology or situation to the patients, hard material model are often sufficient. The low-cost and most accessible method FDM is most certainly the best choice if there is no need for fine printing definition and if the size of the model is large, otherwise we would recommend SLA. Models obtained by SLA present more detail thus would be better for small printing models (coronary arteries).

However, in the case of the thoracic aortic model with root aneurysm we put the emphasis on the realism of the geometry by representing as much as details as possible which is why we needed to use one of the most accurate 3D printing method: PJ. It also allowed us to change easily the colours of the 3D printed model if desired.

2) Surgical planning and review of procedure: Surgical planning and review of procedure do not necessarily require materials to have the same mechanical properties of the biological tissues. Hard material model can be well representative of the anatomical structure and once again, FDM and SLA might be your best options.

3) Pre-procedural planning: Pre-procedural planning models are more complicated to fabricate since they require materials mechanically representative to the biological tissues. Discussions on the matter are provided in the following section where all printing methods are eventually used.

\subsection{Selection of an appropriate use of materials}

The selection of the material is directly linked to the selection of the 3D printing process and printer, as well as the needs of the model. All information developed in the following paragraphs are summarized and reorganized in Table 3.

\subsubsection{Rigid materials}

Human bones are the easiest biological tissues to reproduce by 3D printing as the majority of the materials are rigid. The most common option remains acrylonitrile butadiene styrene (ABS) by FDM (Chan et al., 2015; Cohen \& Reyes, 2015; Mowry, Jammal, Myer, Solares, \& Weinberger, 2015) but powders of plaster (Chan et al., 2015) and hydroquinone (Hochman et al., 2015) were also used by BJ, as well as a mix of polyamide with glass beads by SLS (Suzuki et al., 2004). ABS is the same plastic used in water pipe of most home and is the most affordable material in $3 D$ printing. It has proved to be an appropriate bone 
substitute with good visual and haptic renderings for practicing the drilling with minimized cost, despite the softness of the material (Cohen \& Reyes, 2015). Cohen and Reyes (2015) believed that the benefits of the training will be felt even if the mechanical properties did not match exactly the bone. This study is a perfect example showing that the tissue fidelity does not necessarily need to be completely realistic and that the purposes and the requirements of the models are absolutely essential in the choice of the proper materials.

Rigid materials may also be adequate in the context of pre-procedural planning which has already proven its ability to improve the way surgeons think, interpret, evaluate a procedure and face a complex situation by simulating all steps in advance (Chua, Chou, Lin, Eu, \& Lew, 1998; Gillaspie et al., 2016; Rengier et al., 2010). A lack of imaging (Schmauss et al., 2013) and anatomic consistency (Charcot foot syndrome) (Giovinco et al., 2012), as well as the hard visualisation of a 3D geometry through a 2D monitor (Salloum et al., 2016), might all complicate surgical strategies that can be fulfilled with 3D printed models. The same rigid model can afterwards be used intra-operatively for orientation purposes (Spottiswoode et al., 2013). Moreover, Levi et al. (2002) have highlighted the importance for the residents to see an operation several times to memorize phases of the procedures.

On the other hand, the physical and graspable models help the trainees to familiarize themselves with the human anatomy and unlimited pathologies for a superior understanding (Chae et al., 2015; Deferm et al., 2016), tactile and visual 3D appreciations (Malik et al., 2015; Rengier et al., 2010). Therefore, the accuracy of the procedure will be higher and the diagnostic quality facilitated while the operating time is diminishing up to two thirds (Singhal et al., 2016), as well as the risks of complications and traumas of the patients (Rengier et al., 2010). Models can also be designed with removable structures for a better understanding and visualisations of multitude situations (Soares et al., 2013).

\subsubsection{Flexible materials}

Most of the 3D printing materials present a lack of realism to mimic adequately a soft human biological tissue thus post-processing might be required to soften printed structures. For instance, cartilaginous tissues for dissection and drilling needed a liquid coating to increase the physical strength of a structure created by $\mathrm{BJ}$ in contrast to the infiltrations of elastomeric resins meant to increase its flexibility (Chan et al., 2015). Similarly, BJ was used for tumors in the context of surgical simulation (Kondo et al., 2016), arteries to practice transcatheter valve replacements (Schmauss et al., 2015), as well as hepatic segments (Kong et al., 2016) and hearts (Noecker et al., 2006; Schmauss et al., 2015) to teach human anatomy. 
By contrast, SLA had the capability to fabricate flexible hearts made in urethane suitable for cutting and suturing practices without post-processing (Shiraishi et al., 2010). Similarly, a cartilaginous trachea was also replicated by PJ (Walenga, Longest, \& Sundaresan, 2014) providing a rubber-like material mixable with a rigid photopolymer to control the flexibility of the structure, as arteries (Biglino et al., 2013; Kurenov et al., 2015), soft tissue (K. Wang, Zhao, et al., 2016), mitral valve (Vukicevic et al., 2016) and cerebral aneurysms (Wurm et al., 2011). The trachea, arteries and soft tissues were created to be as realistic as possible while the mitral valve was used to learn catheter-based interventions and to evaluate a surgical device, moreover the aneurysm was to learn how to clip an artery.

\subsubsection{Printing with multiple materials}

Depending on the needs, several materials (colors, properties, textures) may be required to create a proper phantom. Waran et al. (2014) opted for the use of a multi-material PJ machine for a layer-based model made of rigid and soft tissues (bone, dura mater, tumor, normal brain). Similarly, K. Wang, Wu, et al. (2016) created a material made of rigid fibers embedded in a flexible material to control the properties of the printed composite. Chan et al. (2015) manufactured separately the hard structure of the bone and the softer cartilaginous tissues with different materials and processes for a final realistic assembly of a replicated head and neck. Finally, Hochman et al. (2015) added as well three coats of urethane to simulate the dural membrane of the temporal bone models for a better tissue fidelity.

Multi-material composites are the future in the creation of 3D printed models since none of the current available materials can mimic elastic and biological tissues. Hence printing materials containing fibers to control adequately the mechanical behavior of the object are being explored. Mechanical testing can be performed to analyze the biomechanical response of the human tissue by cutting, compressing or tearing apart the material for instance. Emmott et al. (2016) have described the tensile testing method commonly performed in our laboratory to evaluate the biomechanics of the aortic tissues. 
Table 3. 3D printing technologies and materials involved in the fabrication of models for surgical training, their simulated body parts, purposes, requirements and the machines used. Reprinted with permission (Garcia et al., 2017).

\begin{tabular}{|c|c|c|c|c|c|c|c|c|}
\hline & Simulation & Technology & Material & Reproduction & Purpose & Requirement & $\begin{array}{l}\text { Machine/ } \\
\text { Manufacturer }\end{array}$ & Ref \\
\hline \multirow[t]{7}{*}{$\begin{array}{l}\text { Rigid } \\
\text { material }\end{array}$} & \multirow[t]{6}{*}{ Bone } & \multirow[t]{3}{*}{ FDM } & \multirow[t]{3}{*}{ Acrylonitrile butadiene styrene (ABS) } & \multirow[t]{2}{*}{ Temporal bone } & \multirow[t]{2}{*}{ Drilling } & Quick and inexpensive fabrication & & $\begin{array}{l}\text { (Cohen \& Reyes, } \\
\text { 2015) }\end{array}$ \\
\hline & & & & & & $\begin{array}{l}\text { Inexpensive model with good haptic and visual } \\
\text { aspects }\end{array}$ & Makerbot x2 & $\begin{array}{l}\text { (Mowry et al., } \\
\text { 2015) }\end{array}$ \\
\hline & & & & Head and neck & Endoscopic surgery & Durability, accuracy, rigidity, homogeneousity & Vantage/Stratasys & $\begin{array}{l}\text { (Chan et al., } \\
\text { 2015) }\end{array}$ \\
\hline & & \multirow[t]{2}{*}{ BJ } & $\begin{array}{l}\text { Plaster (ZP-130) + Binder (CA101 } \\
\text { Cyanoacrolate) }\end{array}$ & Head and neck & Dissection and drilling & $\begin{array}{l}\text { Anatomical fidelity, cut-ability, realistic } \\
\text { response for drilling }\end{array}$ & $\begin{array}{l}\text { ZP printer } 310 / Z \\
\text { corporation }\end{array}$ & $\begin{array}{l}\text { (Chan et al., } \\
\text { 2015) }\end{array}$ \\
\hline & & & Hydroquinone + Binder (Cyanoacrylate ) & $\begin{array}{l}\text { Cortical and trabecular } \\
\text { temporal bones }\end{array}$ & & $\begin{array}{l}\text { Anatomical fidelity of the internal structure } \\
\text { and proper mechanical characteristics } \\
\text { (elasticity, hardness, vibrations while drilling) }\end{array}$ & $\begin{array}{l}Z \text { printer } 650 / 3 D \\
\text { Systems }\end{array}$ & $\begin{array}{l}\text { (Hochman et al., } \\
\text { 2015) }\end{array}$ \\
\hline & & SLS & Polyamide + Glass beads & Temporal bone & $\begin{array}{l}\text { Drilling, burring and } \\
\text { suction }\end{array}$ & Suitable for surgical simulation & & $\begin{array}{l}\text { (Suzuki et al., } \\
\text { 2004) }\end{array}$ \\
\hline & Tumor & BJ & $\begin{array}{l}\text { Plaster (ZP-150) + Binder (ZB-63 clear) } \\
\text { Wax coating }\end{array}$ & Skull base tumor & $\begin{array}{l}\text { Investigation of the } \\
\text { usefulness of the tumor } \\
\text { by evaluating its } \\
\text { visibility }\end{array}$ & $\begin{array}{l}\text { Tumor with mesh structure suitable for } \\
\text { surgical simulation }\end{array}$ & $\begin{array}{l}Z \text { printer } 450 / 3 D \\
\text { systems }\end{array}$ & $\begin{array}{l}\text { (Kondo et al., } \\
\text { 2016) }\end{array}$ \\
\hline \multirow[t]{2}{*}{$\begin{array}{l}\text { Semi-rigid } \\
\text { material }\end{array}$} & \multirow[t]{2}{*}{ Cartilage } & PJ & Rubber-like resin (TangoPlus FLX930) & Trachea & & $\begin{array}{l}\text { Proper mechanical characteristics } \\
\text { (compliance) }\end{array}$ & \multirow[t]{2}{*}{ Objet500/Connex3 } & $\begin{array}{l}\text { (Walenga et al., } \\
\text { 2014) }\end{array}$ \\
\hline & & BJ & $\begin{array}{l}\text { Plaster (ZP-15) + Binder } \\
\text { Infiltration (Elastomeric resin, 30min) }\end{array}$ & $\begin{array}{l}\text { Septum, middle and } \\
\text { inferior turbinares }\end{array}$ & Dissection and drilling & $\begin{array}{l}\text { Anatomical fidelity, cut-ability, realistic } \\
\text { response for drilling }\end{array}$ & & $\begin{array}{l}\text { (Chan et al., } \\
\text { 2015) }\end{array}$ \\
\hline \multirow[t]{10}{*}{$\begin{array}{l}\text { Flexible } \\
\text { material }\end{array}$} & \multirow[t]{3}{*}{ Artery } & PJ & Rubber-like resin (TangoPlus FLX930) & $\begin{array}{l}\text { Human pulmonary } \\
\text { arteries }\end{array}$ & & & Objet500/Connex3 & $\begin{array}{l}\text { (Kurenov et al., } \\
2015 \text { ) }\end{array}$ \\
\hline & & & & Arteries & & $\begin{array}{l}\text { Proper mechanical characteristics } \\
\text { (distensibility) }\end{array}$ & Objet500/Connex3 & $\begin{array}{l}\text { (Biglino et al., } \\
\text { 2013) }\end{array}$ \\
\hline & & BJ & Model infiltrated with polyurethane & Ascending aorta & $\begin{array}{l}\text { Transcatheter valve } \\
\text { replacement }\end{array}$ & - & $\begin{array}{l}\begin{array}{l}\text { Spectrum } \\
\text { corporation }\end{array} \\
\end{array}$ & $\begin{array}{l}\text { (Schmauss et al., } \\
\text { 2015) }\end{array}$ \\
\hline & Valve & PJ & $\begin{array}{l}\text { Rubber like resin (TangoPlus FLX930 Shore } \\
27 \text { and 35) }\end{array}$ & Mitral valve & $\begin{array}{l}\text { Catheter-based } \\
\text { interventions: Mitraclip } \\
\text { procedure and plugin of } \\
\text { a trans-catheter device }\end{array}$ & $\begin{array}{l}\text { No moving of the MitraClip after pulling and } \\
\text { accuracy of the geometry }\end{array}$ & Objet500/Connex3 & $\begin{array}{l}\text { (Vukicevic et al., } \\
\text { 2016) }\end{array}$ \\
\hline & \multirow[t]{4}{*}{ Heart } & \multirow[t]{3}{*}{ BJ } & $\begin{array}{l}\text { Plaster (ZP 150) + Binder (Z-bond 90) } \\
\text { Plaster (ZP 150) + Binder (N/A) }\end{array}$ & Hepatic segments & & $\begin{array}{l}\text { Realism of anatomical conditions, quality, } \\
\text { color, and tactility }\end{array}$ & $\begin{array}{l}\begin{array}{l}\text { Spectrum } \\
\text { corporation }\end{array} \\
\end{array}$ & $\begin{array}{l}\text { (Kong et al., } \\
\text { 2016) }\end{array}$ \\
\hline & & & $\begin{array}{l}\text { Starch/cellulose + Binder (Polymer) } \\
\text { Infiltration (urethane) }\end{array}$ & & & & $\begin{array}{l}\begin{array}{l}\text { Spectrum } \\
\text { corporation }\end{array} \\
\mathrm{Z} 5 \mathrm{~s} 10 / \mathrm{Z}\end{array}$ & $\begin{array}{l}\text { (Schmauss et al., } \\
\text { 2015) }\end{array}$ \\
\hline & & & $\begin{array}{l}\text { Starch-based powder } \\
\text { Elastomer coating }\end{array}$ & & & & & $\begin{array}{l}\text { (Noecker et al., } \\
\text { 2006) }\end{array}$ \\
\hline & & SLA & Urethane & & Cutting and suturing & & & $\begin{array}{l}\text { (Shiraishi et al., } \\
\text { 2010) }\end{array}$ \\
\hline & $\begin{array}{l}\text { Cerebral } \\
\text { aneurysm }\end{array}$ & PJ & Rubber-like resin & Cerebral vessel & Clipping the aneurysm & Proper mechanical characteristics & Objet500/Connex3 & $\begin{array}{l}\text { (Wurm et al., } \\
\text { 2011) }\end{array}$ \\
\hline & Soft tissue & PJ & $\begin{array}{l}\text { Rigid material (VeroBlackPlus RGD875) + } \\
\text { Rubber-like resin (Tangoplus FLX930) }\end{array}$ & & & Proper mechanical characteristics & Connex350 & $\begin{array}{l}\text { (K. Wang, Zhao, } \\
\text { et al., 2016) }\end{array}$ \\
\hline
\end{tabular}


Skin, bone, PJ

dura mater,

PJ

tumor, brain
Cutting and suturing Phantom providing the haptic of a surgery with Objet500/Connex3 layers made of different materials

(Waran et al.,

(skin); Perforation and

cutting (bone); Drilling 


\subsection{Discussion}

We described the main steps of a general and straightforward method to follow for the creation of 3D printed patient-specific models for medical education based on the model of a thoracic aorta with root aneurysm (Figure 27): teaching the anatomy, planning a surgery or practicing procedures and medical manipulations as the head model to practice the drilling in Figure 28 created by Chan et al. (2015) with one material or in Figure 29 with a combination of materials by Waran et al. (2014). We started by listing the most appropriate choice of imaging technology to visualize the geometry depending on the tissues and explained how to convert the information to a STL 3D geometrical object for fabrication.

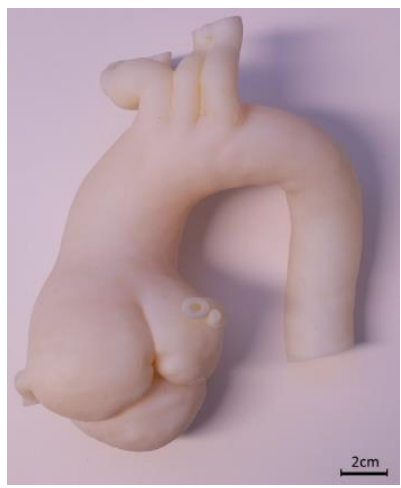

Figure 27. Model of the aorta with root aneurysm for teaching purposes. Reprinted with permission (Garcia et al., 2017).

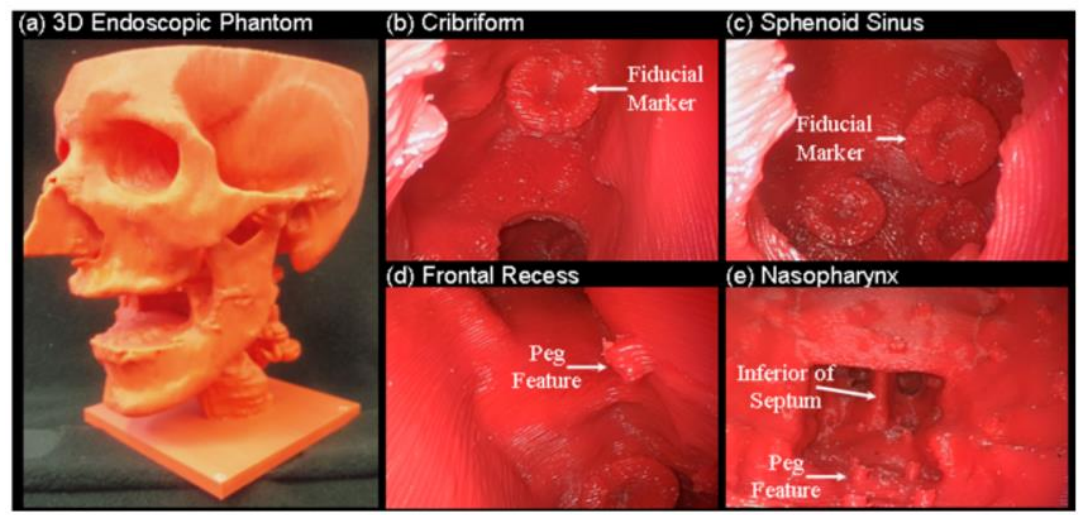

Figure 28. Head model made of one material to practice the drilling in medical simulation (Chan et al., 2015). Reprinted with permission (Garcia et al., 2017). 


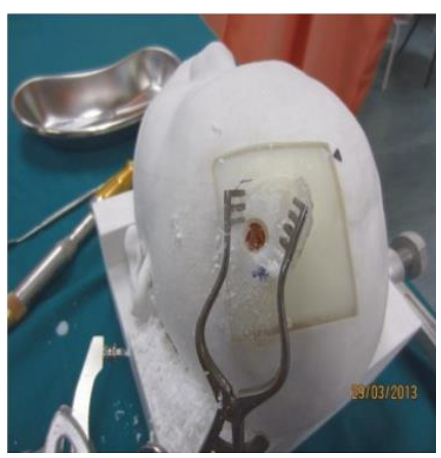

Figure 29. Head model made of a combination of materials to practice the drilling in medical simulation Waran et al. (2014). Reprinted with permission (Garcia et al., 2017).

Medical imaging gives good anatomic spatial resolution but still need to be improved in some areas such as the aortic valves. In addition, the automatic segmentation is not optimal, cannot be entirely trusted (artifacts may not be detected or good part of the geometry may be identified as undesired) and is not good as manual segmentation. Creating a 3D geometrical model requires a high level of anatomical knowledge and coordination between surgeon, radiologist, and engineer. It is a relatively simple concept but does require specific multidisciplinary expertise. There is not a "one size fits" all in this growing field and educational goals, required technical expertise, and cost are important considerations when starting a 3D printing program. However, a good understanding of the 3D printing process will greatly help you meet your educational goals. A recent study has shown the possibility to fabricate very accurate 3D aortic models (c.f. intimal flap) with a $1 \mathrm{~mm}$ of error in the mean difference in luminal diameter (Ho \& Sun, 2017) which would allow an efficiency improvement by its use in medical simulation (see Figure 30).
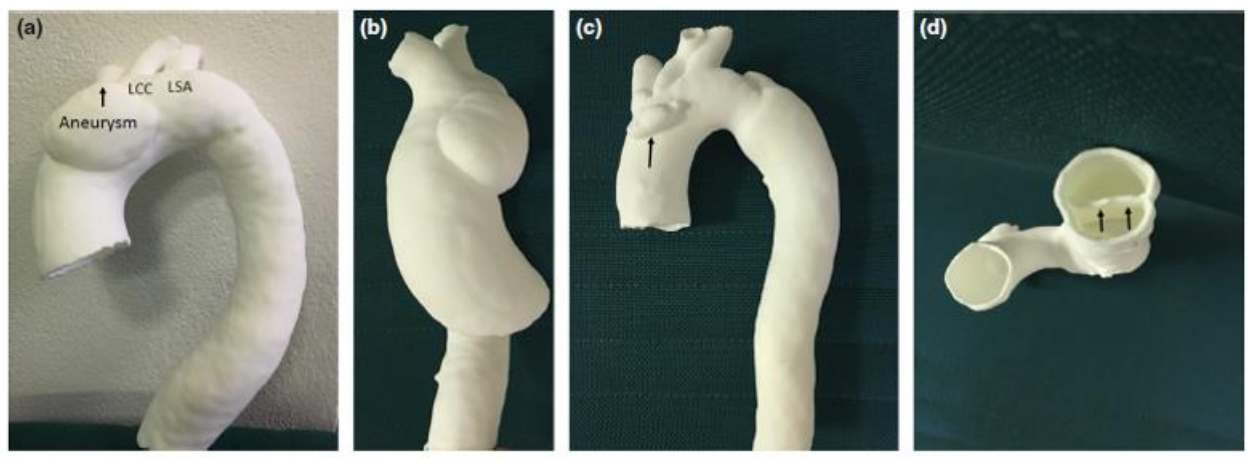

Figure 30. Accurate 3D model of an aortic aneurysm captured from CT imaging (Ho \& Sun, 2017). Reprinted with permission (Garcia et al., 2017). 
3D printing is an attractive, powerful, versatile technology which has the potential to be very accessible to anyone who would happen to be interested. It is gaining popularity mostly in orthopedics, dentistry and plastic surgery where bones are easier to replicate, even though much work is needed in this area. 3D printed specific-patient models have demonstrated that they can increase performance and foster rapid learning (Rosen, 2008) while significantly ameliorating the knowledge, management and confidence of the trainees regardless of the area of expertise (Chakravarthy et al., 2011). Physical interaction has been proven to be the key to gaining motor skills needed for surgical intervention improving operating room outcomes (Armillotta et al., 2007; Kalejs \& von Segesser, 2009; Sulaiman et al., 2008; Suzuki et al., 2004).

Nevertheless, very few materials currently present elastic properties ideal for surgical training in the field of cardiac surgery for instance. This is the current main limitation that the companies and researchers are trying to solve and even if there is a progress on the matter, soft materials such as silicone can already be printed but are not suitable for complex geometries (Oxman et al., 2012). In the near future, we believe that the most representative printed materials to mimic soft tissues would have to be made of several components. Manipulating the materials themselves as an area of research and innovation will widen the possibilities thus improve the mechanical responses of the printed models and provide better teaching tool for surgical education. A combination of materials in 3D printing have recently been explored by Waran et al. (2014), as well as in our lab where we tend to replicate the natural structure of the biological aortic tissue to mimic as far as possible its biomechanical properties.

Printing materials were selected to create a novel flexible composite which simulates the composition of the aortic tissue by representing its smooth muscle cells with a non-mechanically responsive material and the matrix of proteins with a rigid one. Mechanical tests were performed for comparison purposes and resemblances with the biological material have been found. Hopefully this process and research will open a new future for 3D printing. One which will not only include anatomical fidelity but tissue fidelity as well.

Finally, we believe that 3D digital reconstruction of surgical anatomy is complimentary to 3D physical models. Shah et al. (2017) studied the importance of the variation in teaching for undergraduate dental education and found that the majority of students had a preference for kinesthetic learning (tactile learning). Those physical interactions or activities are the reasons why 3D printed models are essential in the training of medical professionals. 


\section{Chapter 5: Paper 2}

\subsection{Preface}

The following manuscript was published in 2018 in the Journal of Surgical Simulation: Garcia, J., M. AlOmran, A. Emmott, R. Mongrain, K. Lachapelle, and R. L. Leask. 2018. Tunable 3D printed multi-material composites to enhance tissue fidelity for surgical simulation, Journal of Surgical Simulation, 5: 87-98.

In the previous manuscript, we developed a method to 3D print patient-specific aortic models but only used rigid materials. The presented work focused on the creation of heterogeneous and tunable composites by 3D printing (Polyjet technology) for aortic tissue-mimicking phantoms in the context of surgical training. We wanted to obtain a 3D printable and visco-elastic material that will eventually be implemented in patient-specific models to enhance the surgical simulation.

We chose to replicate the natural structure of the vessel for the creation of the 3D printed tissue before testing the materials for comparison purposes (Objective 1.1.). Effects of the embedded fibers to the composite and variations in its structure were also investigated (Objective 1.2.).

We found that three-material composites were able to reproduce mechanical properties of human ascending thoracic aortas that were previously collected from surgeries or autopsies (mechanical and physiological fidelities corresponding to the Objectives 2.2 and 2.3 respectively). In addition, changing the structure as well as the fibers geometry affected the mechanical properties of the composites (elasticity, strength and directional dependency) (Objective 1), thus improved the current homogeneous 3D printed TGPF930 material often used in surgical education.

Complementary information about the data analyses in tensile testing is presented in Appendix 1. Appendix 2 gives more details about the chemical compositions and properties of the materials after thermogravimetric analysis (TGA) and differential scanning calorimetry (DSC). 
Tunable 3D printed multi-material composites to enhance tissue fidelity for surgical simulation

Justine Garcia ${ }^{1}$, Mansour AlOmran ${ }^{2}$, Alexander Emmott ${ }^{3}$, Rosaire Mongrain ${ }^{1}$, Kevin Lachapelle ${ }^{2}$, Richard L. Leask ${ }^{3 *}$

(1) Mechanical Engineering, McGill University, Montreal, Quebec, Canada

(2) Cardiovascular Surgery, McGill University Health Network, Montreal, Quebec, Canada

${ }^{(3)}$ Chemical Engineering, McGill University, Montreal, Quebec, Canada

* corresponding author

Keywords: Surgical simulation; 3D printing; Simulation; Ascending aortic tissue; Biomechanics; 3D printed composite

\subsection{Abstract}

Medical simulation is an important component in surgical education. Unfortunately, there are very few 3D printed materials that have the tissue fidelity needed for enhanced learning of cardiovascular surgical techniques. Therefore, we sought to determine if we could develop 3D printed composites to better reflect the tissue mechanical properties of the ascending aorta.

3D printed composites were created using commercially available materials for a Connex3 Objet500 3D printer (Stratasys, Eden Prairie, USA). Support material (SUP705) as well as rigid zigzag Vero fibers were systematically combined with an elastic polymer (TGPF930). The mechanical properties were evaluated in equi-biaxial tensile (tensile stiffness and visco-elasticity), nano-indentation (compressive stiffness) and suture retention strength (strength) (SRS) tests for comparisons to normal ascending thoracic aortas (ATAA) and ascending aortas with aneurysm (ATAA).

When compared to TGPF930 (Unpaired t-test), the insertion of support material reduced the SRS (TGPF930: 4.45 $\pm 0.49 \mathrm{~N}, \mathrm{~N}=3$;TGPF930 + SUP705: $2.57 \pm 0.32 \mathrm{~N}, \mathrm{~N}=3 ; \mathrm{p}=0.0023$ ) and compressive modulus (TGPF930: 0.61 $\pm 0.08 \mathrm{MPa}, \mathrm{N}=6$; TGPF930 + SUP705: 0.38 $\pm 0.06 \mathrm{MPa}, \mathrm{N}=6 ; \mathrm{p}=0.0002$ ). Embedding Vero fibers increased the SRS (TGPF930: $4.45 \pm 0.49 \mathrm{~N}, \mathrm{~N}=3$; TGPF930 + Vero: $5.39 \pm 0.65 \mathrm{~N}, \mathrm{~N}=3 ; \mathrm{p}=0.0037$ ), 
compressive modulus (TGPF930: $0.61 \pm 0.08 \mathrm{MPa}, \mathrm{N}=6$; TGPF930 + Vero: $0.85 \pm 0.07 \mathrm{MPa}, \mathrm{N}=6 ; \mathrm{p}=0.0003$ ) and allowed for tuning the mechanical directional dependency of the composite. When all three components were combined, similarities were found with aortic tissue in terms of SRS (three-material composite: $4.25 \pm 0.67 \mathrm{~N}, \mathrm{~N}=3$; ATAA: $5.31 \pm 2.71 \mathrm{~N}, \mathrm{~N}=3 ; \mathrm{p}=0.6177$ ) and compressive modulus (threematerial composite: $0.39 \pm 0.03 \mathrm{MPa}, \mathrm{N}=6$; ATAA: $0.36 \pm 0.12 \mathrm{MPa}, \mathrm{N}=4 ; \mathrm{p}=0.4485$ ).

The study has shown that the insertions of fibers and/or support material in a TGPF930 structure can control the mechanical properties of the 3D printed composites. We were able to simulate ex vivo passive tissue characteristics of aortas, therefore improving on the current homogeneous 3D printed TGPF930 material often used in surgical education.

\subsection{Introduction}

Simulation is an important tool in medical education as it has been shown to enhance the learning and acquisition of basic technical skills outside the operating room (Malik et al., 2015). Surgical simulators are often made with synthetic material (latex, silicone) which acts as a surrogate for human tissue. Such surgical simulators lack the anatomic, tissue and physiologic fidelity required for advanced surgical training. Biological tissue, such as animal and cadaveric material, provide excellent tissue fidelity, however they are expensive, lack patient specific pathologies and have limited physiological realism. For these reasons, we sought to determine if we could develop a 3D printing strategy to better reflect the anatomical, physiological and tissue fidelity needed for surgical simulation.

Ascending aortas (AA) are frequently involved in cardiothoracic procedures where they are manipulated, canulated, cut, sewed and resected or reconstructed by surgeons. The vessel is very elastic and naturally designed to absorb the energy of systole and redistribute it through visco-elastic recoil during diastole (Windkessel effect). Pathologies of the ATA greatly change the mechanical properties and function of the vessel. With disease, the ATA stiffens, increases energy loss and loses its mechanical directional dependency (Chung et al., 2017; Shahmansouri et al., 2016). The energy loss is the amount of energy absorbed by the vessels during cyclic loading and is therefore a measure of the visco-elasticity of the tissue that estimates the ability of the material to perform a Windkessel function. Therefore, controlling the material properties of synthetic aortic tissues for realistic training models is important as it would provide trainees with the haptics of a large panel of potential pathologies. 
This study aims to describe our process and technique for creating 3D printed material composites with variable and controllable mechanical properties to simulate aortic walls. Given the heterogenous nature of the ATA and its tendency to change its mechanical characteristics in diseased states, we elected to approach the problem of tissue fidelity by employing a fibrous multi-material composite using commercially available products. The material construct properties were compared and validated with ex vivo passive normal and abnormal freshly harvested aortic tissue.

\subsection{Materials and methods}

\subsubsection{Creation of the heterogeneous synthetic aortic tissue}

To guide the development of our synthetic tissues, we first evaluated a flexible 3D printable material Tangoplus FullCure 930 (TGPF930) in which brittle support material (SUP705) and rigid Verowhite (Vero) were added to change the properties of the structure. All resins were available for use with a Connex 3 Objet500 (Stratasys, Eden Prairie, USA). Pure TGPF930 has previously been used to 3D print synthetic arteries (Biglino et al., 2013; Kurenov et al., 2015), mitral valves (Vukicevic et al., 2016) and cerebral aneurysms (Wurm et al., 2011). It is relatively elastic, homogenous and isotropic when printed by Polyjet technology, so limited in its ability to recreate different tissue pathologies. We used TGPF930 as a base material to create two- and three-material composites to tune the mechanical properties of our synthetic aortic tissue. The steps can be described as follows (Figure 31):

- To soften a TGPF930 structure, we inserted brittle support material (SUP705);

- To strengthen a TGPF930 structure, we embedded two layers of rigid Vero fibers;

- To control the mechanical directional dependency, we embedded one layer of large fibers;

- To create a tunable, soft but strong composite from a TGPF930 structure, we embedded two layers of rigid Vero fibers and inserted brittle support material (SUP705).

Preliminary studies demonstrated that the size, volume density (distance between the fibers, number of layers of fibers) and shape of the fibers (pattern, amplitude and phase) have a significant impact on the stiffness of the composite material (Yang, 2016). We therefore chose a series of candidate composites based on these preliminary studies to demonstrate how the tissue fidelity can be tuned to create synthetic ATA tissue. 
Control of the material softness

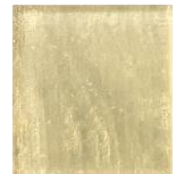

TGPF930

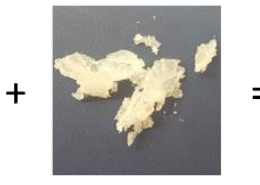

SUP705

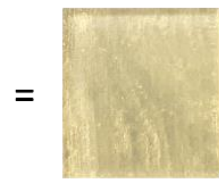

Control of the material strength

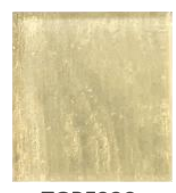

TGPF930

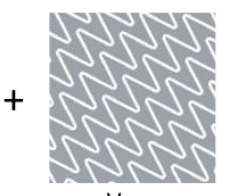

Vero

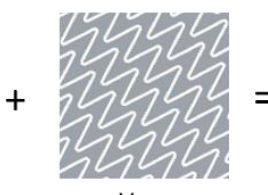

Vero

Control of the material directional dependency

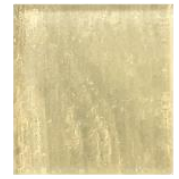

TGPF930

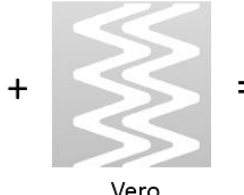

Vero

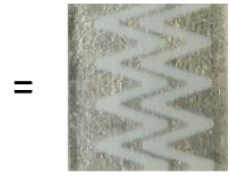

Tunable, soft but strong three-material composite

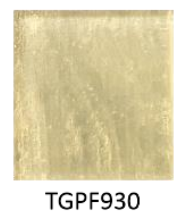

TGPF930

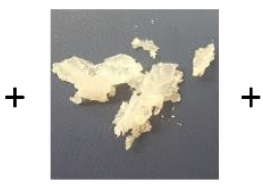

SUP705

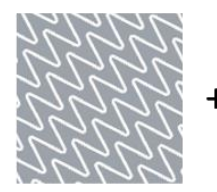

Vero

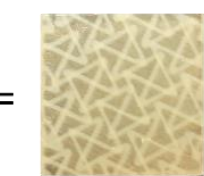

Figure 31. Composition and materials used to control the softness, strength and directional dependency of soft TGPF930 structures, as well as a tunable, soft but strong three-material composite. Reprinted with permission

\subsubsection{Mechanical evaluations of the materials}

Each 3D printed sample was tested within $48 \mathrm{~h}$ of fabrication. Similarly, all samples were printed with the same printer and tested by the same operator, conditions and with fully defined standard operating procedures. The number of 3D printed samples was limited and the distribution is assumed to be normal. Moreover, all samples were printed with the same batch of raw materials.

All aortic tissue was collected from transplant donors and patients undergoing surgery at the Royal Victoria Hospital (Montreal, Quebec, Canada) in accordance with the tri-council Ethical Conduct for Research Involving Humans. Three mechanical tests were carried out to assess the ability to tune the mechanical behavior of the 3D printed composites (tests and outcomes in Table 4). The results are compared with normal and aneurysmal ex vivo passive tissue characteristics of human ATA samples. Planar equi-biaxial tensile testing was used to reveal material abilities to deform and stretch (Emmott et al., 2016). In addition, nano-indentation testing (ASTM E2546) (also-known-as a compression test) was 
used to evaluate the stiffness in compression, thus the compressive modulus important for the haptics on a macro level and tactile properties of the material. Finally suture retention strength (SRS) assessed the material's ability to be sewed for suturing practice.

Table 4. Mechanical tests and outcomes obtained from the study.

\begin{tabular}{ll}
\hline Test & Outcome \\
\hline Equi-biaxial tensile test & $\begin{array}{l}\text { Apparent elastic modulus (tensile stiffness: stiff/ soft) and energy loss (visco- } \\
\text { elasticity: inefficient/efficient Windkessel function) }\end{array}$ \\
\hline Nano-indentation test & Compressive modulus (compressive stiffness: stiff/soft) \\
\hline Suture retention strength test & Forces to tear the material (strength: tough/ fragile) \\
\hline
\end{tabular}

5.4.3. Apparent elastic modulus and energy loss of the material: equi-biaxial tensile testing Equi-biaxial tensile testing was carried out within $24 \mathrm{~h}$ of the excision. Samples of human tissue or composite material $\left(15 \times 15 \mathrm{~mm}^{2}\right)$ were subjected to equi-biaxial stretching at constant strain rate $(0.1 \mathrm{~mm} / \mathrm{s})$ (ELF 3200 , Bose Co., Framingham, USA) in a bath at $37^{\circ} \mathrm{C}$. The engineering stress and strain of the loading curves were fit to a second order Mooney-Rivlin model (Pazos et al., 2009). This model was used to calculate the apparent elastic modulus in MPa (commonly called stiffness in tensile testing, calculated with the slope of the loading curve) at 25\% strains (Figure 32 (a)). The percentage of energy lost over the testing cycle was computed with the raw data to quantify the visco-elastic nature of the ATA and 3D printed composites. The loading and unloading stress-strain curves of the aortic tissue formed a hysteresis loop as a result of a storage and release of energy (Chung et al., 2014) (Figure 32).

\subsubsection{Compressive modulus: nano-indentation testing (ASTM E2546)}

Compressive tests were performed with the Nanovea M1 nano-indenter (Nanovea, Irvine, USA) as per the ASTM E2546 (Standard Practice for Instrumented Indentation Testing) with a 1mm diameter spherical tip to assess the compressive stiffness of the material with a compressive modulus ( $E_{\text {comp }}$ in $\mathrm{GPa}$ ). $\mathrm{E}_{\text {comp }}$ defined in the Oliver-Pharr method (Oliver \& Pharr, 1992) helps to determine the tactile properties of the materials thus their abilities to be squished. The parameter is calculated from the Poisson's ratio of the material ( $v$ is assumed 0.5$)$, the apparent elastic modulus and Poisson's ratio of the indenter $\left(E_{\text {ind }}=200 G P a\right.$, $\left.v_{\text {ind }}=0.1\right)$, as well as the reduced modulus $E_{r}($ in $G P a)$.

$E_{\text {comp }}=\frac{\left(1-v^{2}\right) E_{\text {ind }} E_{r}}{E_{\text {ind }}-E_{r}\left(1-v_{\text {ind }}^{2}\right)}$ 
The $E_{r}$ is derived from the material stiffness obtained from compression $\left(S_{i}\right.$ in $\left.m N / \mu m\right)$ and projected contact area $\left(A_{c}\right.$ in $\left.\mu m^{2}\right)$ of the spherical tip. $A_{c}$ is expressed with the radius of the sphere $(R$ in $\mu m)$, the contact depth ( $h_{c}$ in $\mu m$ ) computed with the maximum depth penetration ( $h_{\max }$ in $\left.\mu m\right)$, the maximum load $\left(P_{\max }\right.$ in $\left.\mathrm{mN}\right)$ and stiffness corresponding to the slope of the beginning of the unloading curve (Figure 32).

$E_{r}=\frac{\sqrt{\pi} s_{i}}{2 \sqrt{A_{c}}}$

$S_{i}=\frac{d P}{d h}$

$A_{c}=2 \pi R h_{c}$

$h_{c}=h_{\max }-\frac{3 P_{\max }}{4 S_{i}}$

\subsubsection{Suture retention strength testing}

SRS is defined the maximum force in $\mathrm{N}$ required to tear the suture from the material. The evaluations were performed with a $500 \mathrm{~N}$ vertical tensile tester EZ Test (Shimadzu, Columbia, USA) at room temperature with a single 4-0 prolene suture. The samples were pulled at $20 \mathrm{~mm} / \mathrm{min}$ (Figure 32 (c)). Triplicates of each synthetic aorta material were tested and compared with ATA tissue samples.

\subsubsection{Statistical analyses}

Data are presented as mean \pm standard deviation and the analyses were performed using GraphPad Prism 5 (GraphPad Software, Inc., La Jolla, USA). Comparisons between groups were done with Paired and Unpaired t-tests. A p value of 0.05 was taken as significant. The results are summarized in Table 5. 
(a) Equi-biaxial tensile test
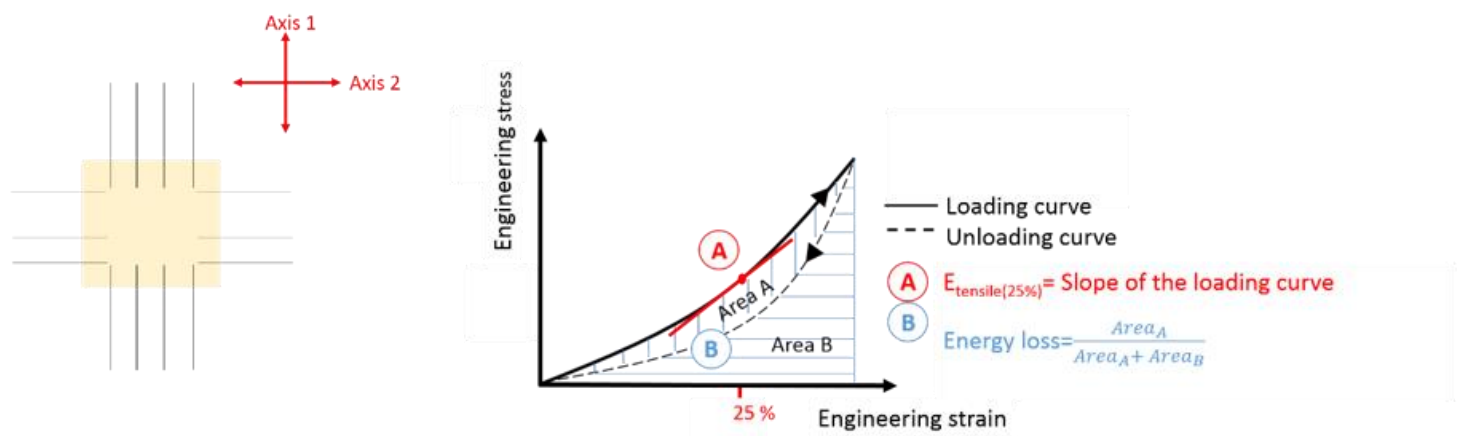

(b) Nano-indentation test
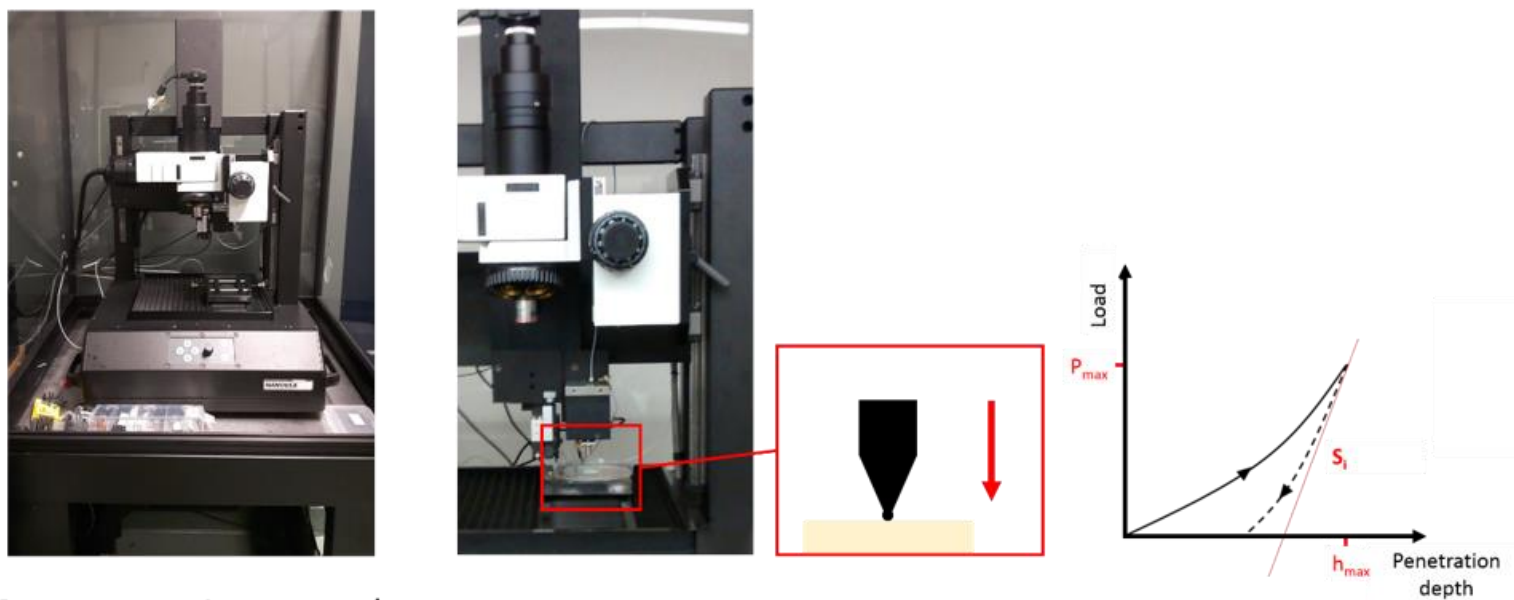

(c) Suture retention strength test
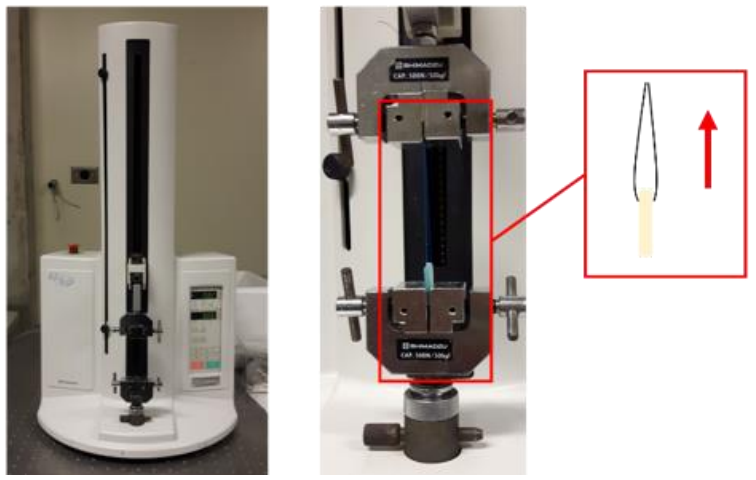

Figure 32. a) Equi-biaxial tensile test: tensile tissue tester stretching the sample and resulting stress-strain curve to evaluate the

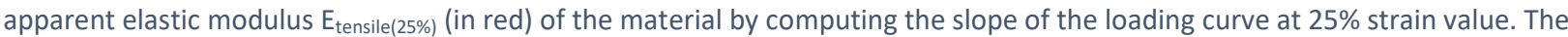
energy loss was calculated by using the A and B areas, b) nano-indentation test: typical loading and unloading curves of the load and penetration-depth with visco-elastic materials in indentation. $\mathrm{P}_{\max }$ is the maximum load, $\mathrm{h}_{\max }$ the maximum depth and $\mathrm{Si}$ the stiffness of the material calculated with the slope of the beginning of the unloading curve. Nanovea M1 nano-indenter

(Nanovea, Irvine, USA) during a test using a spherical tip moving down and touching the surface of the sample, c) suture retention strength: Tensile tester EZ Test (Shimadzu, Columbia, USA) and a suture retention strength test with a 4-0 prolene suture. 


\subsection{Results}

\subsubsection{Evaluation of the aortic tissue}

ATA were collected at the time of surgery. Pathologic specimens were obtained from patients with an ATAA. Normal specimens were obtained from donor hearts harvested for transplants. The apparent elastic modulus (computed at $25 \%$ strains) for normal aortas ranging from $0.05 \mathrm{MPa}$ to $0.09 \mathrm{MPa}$ $(0.08 \pm 0.01 \mathrm{MPa}$ and $0.06 \pm 0.01 \mathrm{MPa}$ for the circumferential and axial directions, $\mathrm{N}=4$ respectively). The normal energy loss of $20 \%$ to $29 \%$ ( $26 \pm 3 \%$ and $23 \pm 3 \%$ for the circumferential and axial directions respectively). The compressive modulus of the normal donors ranged from $0.26 \mathrm{MPa}$ to $0.51 \mathrm{MPa}$ $(0.36 \pm 0.12 \mathrm{MPa}, \mathrm{N}=4)$. Aneurysmal aortas had an apparent elastic modulus that ranged from $0.05 \mathrm{MPa}$ to $0.11 \mathrm{MPa}(0.09 \pm 0.02 \mathrm{MPa}$ and $0.07 \pm 0.02 \mathrm{MPa}$ for the circumferential and axial directions, $\mathrm{N}=19$ respectively) and an energy loss from $23 \%$ to $36 \%$ ( $31 \pm 5 \%$ and $28 \pm 5 \%$ for the circumferential and axial directions respectively). The aneurysmal tissue compressive modulus ranged between $0.15 \mathrm{MPa}$ and $0.36 \mathrm{MPa}(0.29 \pm 0.12 \mathrm{MPa}, \mathrm{N}=3)$. Finally, the forces required to tear sutures from the aneurysmal ATA were between $3.09 \mathrm{~N}$ and $8.33 \mathrm{~N}$ in circumferential direction $(5.31 \pm 2.71 \mathrm{~N}, \mathrm{~N}=3)$, as well as $2.38 \mathrm{~N}$ and $5.93 \mathrm{~N}$ in axial direction $(3.59 \pm 2.03 \mathrm{~N}, \mathrm{~N}=3)$.

\subsubsection{Evaluation of the soft 3D printed material}

We evaluated TPF930 by itself and compared the material to ATA (Unpaired t-tests) (Figure 33). TGPF930 had a similar SRS to the aneurysmal tissue $(4.45 \pm 0.49 \mathrm{~N}, \mathrm{~N}=3, \mathrm{p}=0.5004)$. However, the printing material was stiffer and more viscous than ATA $(p<0.05)$ due to its higher apparent elastic modulus $(0.17 \pm 0.02 \mathrm{MPa}$, $\mathrm{N}=3$ ), compressive modulus $(0.61 \pm 0.08 \mathrm{MPa}, \mathrm{N}=6)$ and energy loss ( $39 \pm 6 \%, \mathrm{~N}=3$ ). Consequently, TGPF930 is stiffer and harder than human aortic tissues. 
(a)

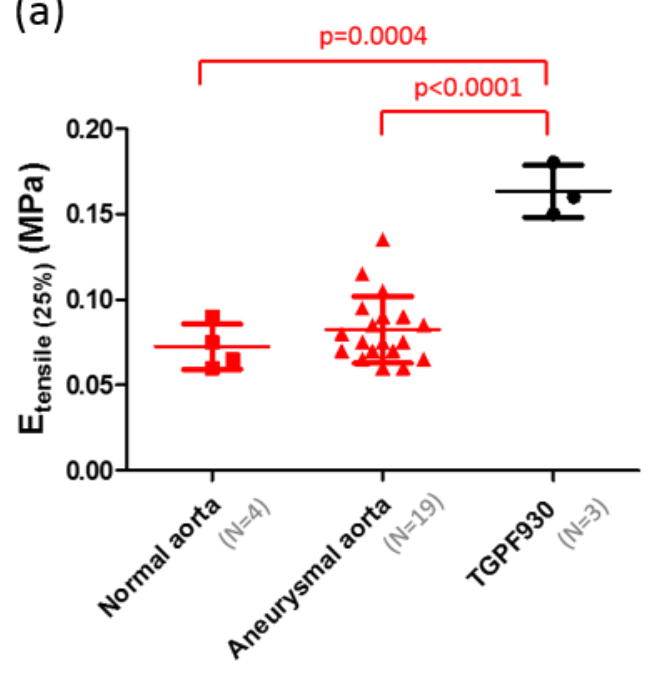

(c)

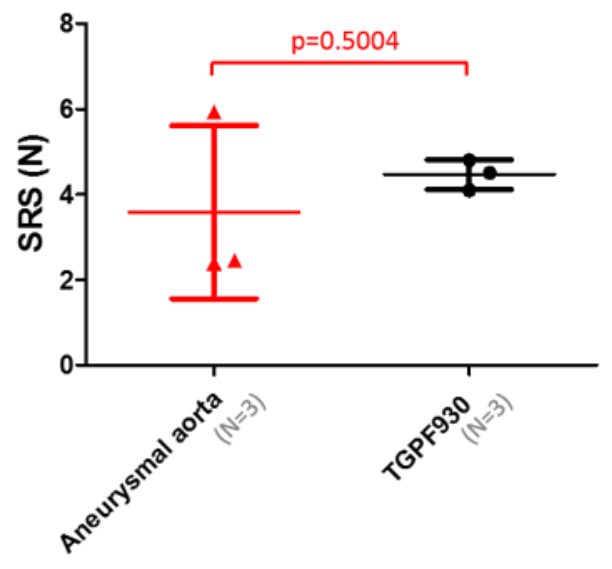

(b)

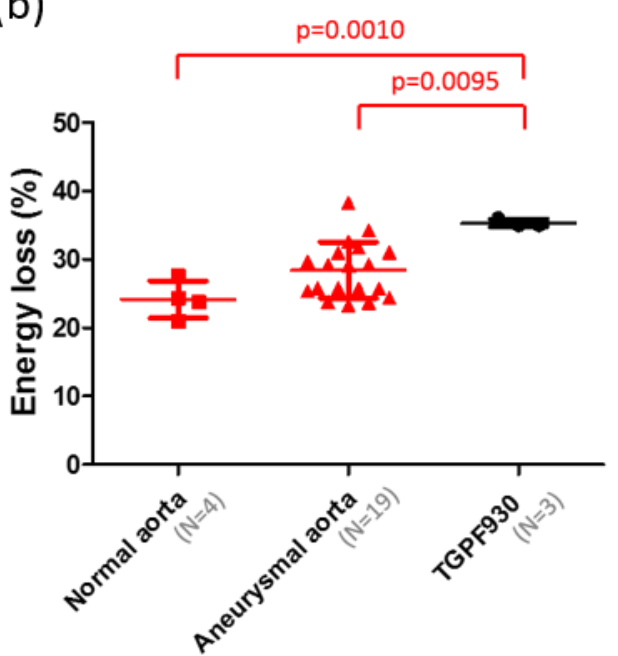

(d)

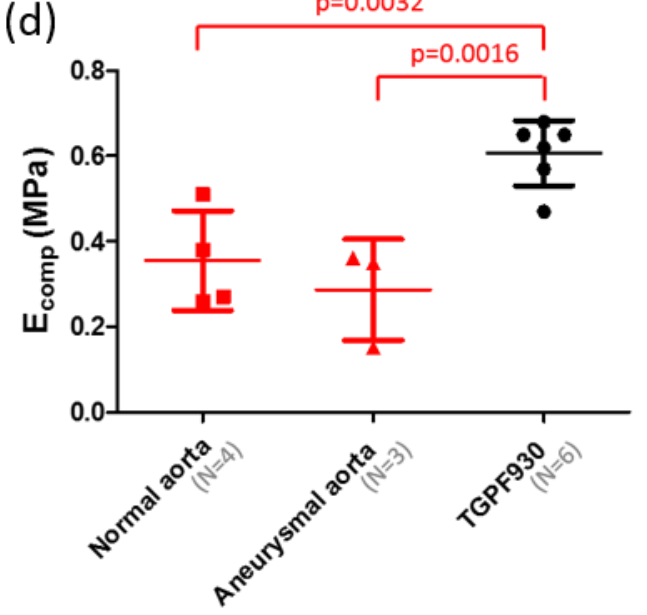

Figure 33. a) The apparent elastic modulus ( $\left.E_{\text {tensile }(25 \%)}\right)$, b) energy loss, c) suture retention strength (SRS) and d) compressive modulus ( $E_{\text {comp }}$ ) of pure Tangoplus FullCure 930 (TGPF930) and aortic tissues (normal and aneurysmal). p values of Unpaired ttests.

\subsubsection{Control of the 3D printed material softness}

The insertion of support material (SUP705) in soft TGPF930 structure significantly softened the composite but made it too brittle. In fact, it was impossible to test the equi-biaxial tensile properties of these twomaterial composites because every sample ripped during the experiments. In addition, it significantly decreased the SRS by $42 \%$ (TGPF930 + SUP705: $2.57 \pm 0.32 \mathrm{~N}, \mathrm{~N}=3, \mathrm{p}=0.0023$ ) and the compressive modulus by $38 \%$ (TGPF930 + SUP705: 0.38 $\pm 0.06 \mathrm{MPa}, \mathrm{N}=6, \mathrm{p}=0.0002$ ) when compared to pure TGPF930 (Figure 34). Therefore, adding the brittle component (SUP705) alone to the rubber material changes the overall 
mechanical response of the structure but made the samples too brittle to be used as synthetic aortic tissue.

(a)

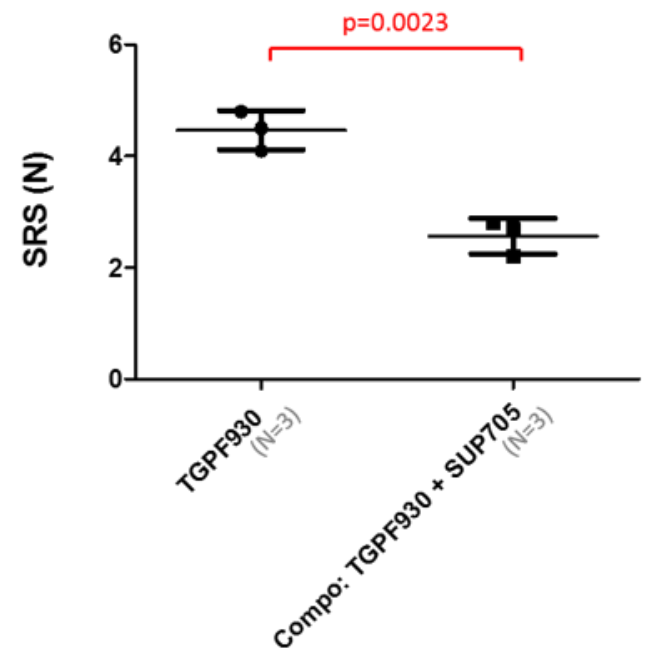

(b)

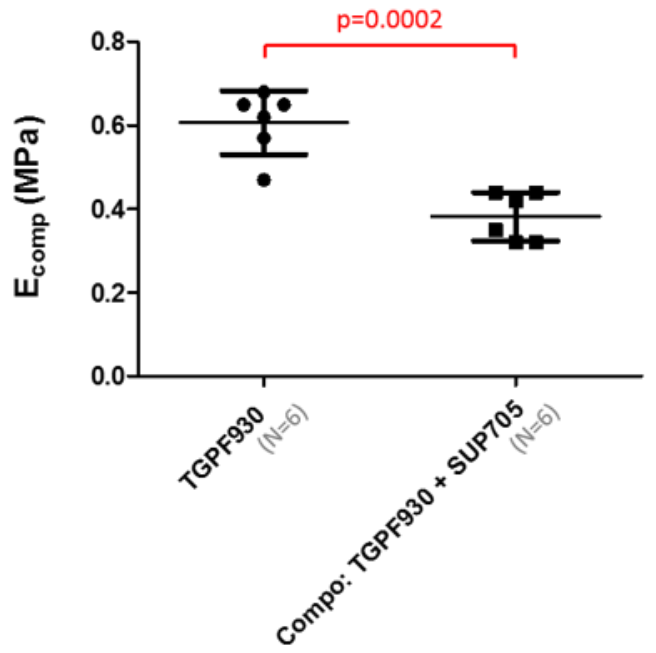

Figure 34. a) The suture retention strength (SRS) and (b) compressive modulus ( $E_{\text {comp }}$ ) of pure Tangoplus FullCure 930 (TGPF930) and two-material composite (Tangoplus FullCure 930 (TGPF930) as well as support material (SUP705)). p values of Unpaired ttests.

\subsubsection{Control of the 3D printed material strength}

Two layers of rigid Vero fibers were embedded in a TGPF930 structure to reproduce the extracellular matrix structure of the arteries and strengthen the material. When compared to pure TGPF930 (Unpaired t-test), the fibers increased the energy loss $(43 \pm 1 \%, N=3, p<0.0001)$, SRS (difference of $21 \%)(5.39 \pm 0.65 N$, $\mathrm{N}=3, \mathrm{p}=0.0037)$ and compressive modulus (difference of $39 \%)(0.85 \pm 0.07 \mathrm{MPa}, \mathrm{N}=6, \mathrm{p}=0.0003)$ (Figure 35). Embedding layers of rigid Vero fibers in flexible TGPF930 structure affects significantly the energy loss, SRS and compressive modulus. 
(a)
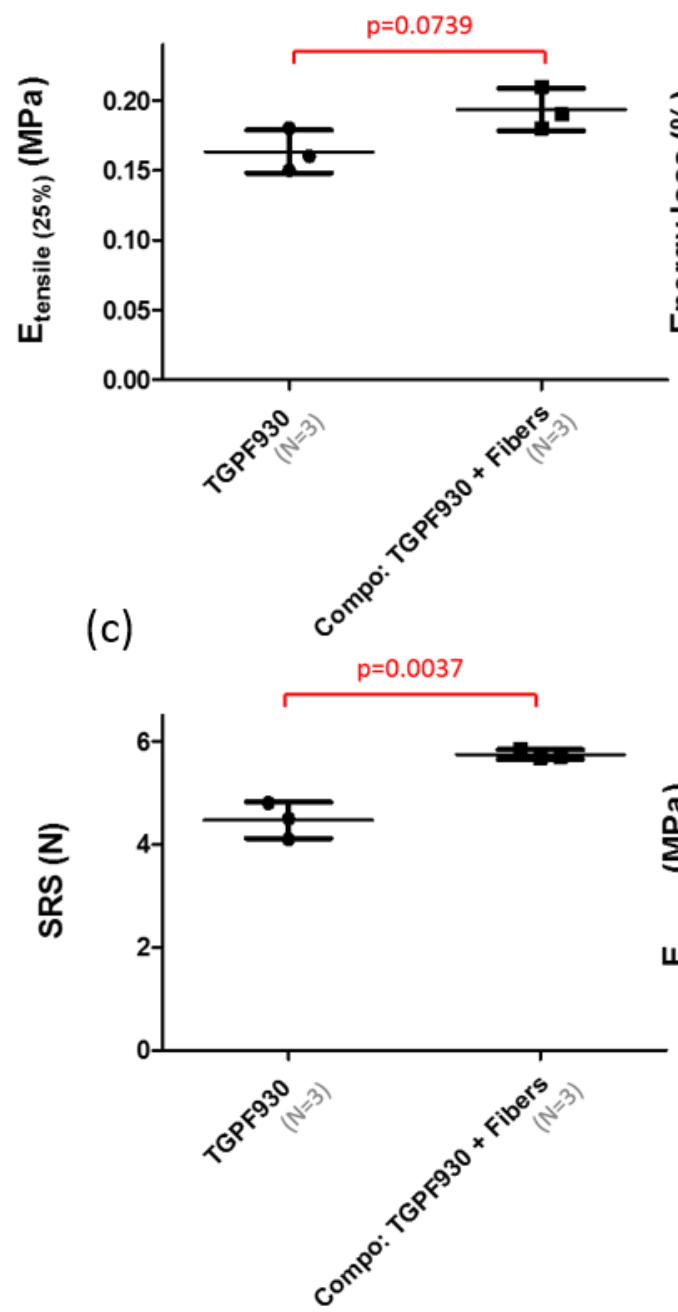

(b)
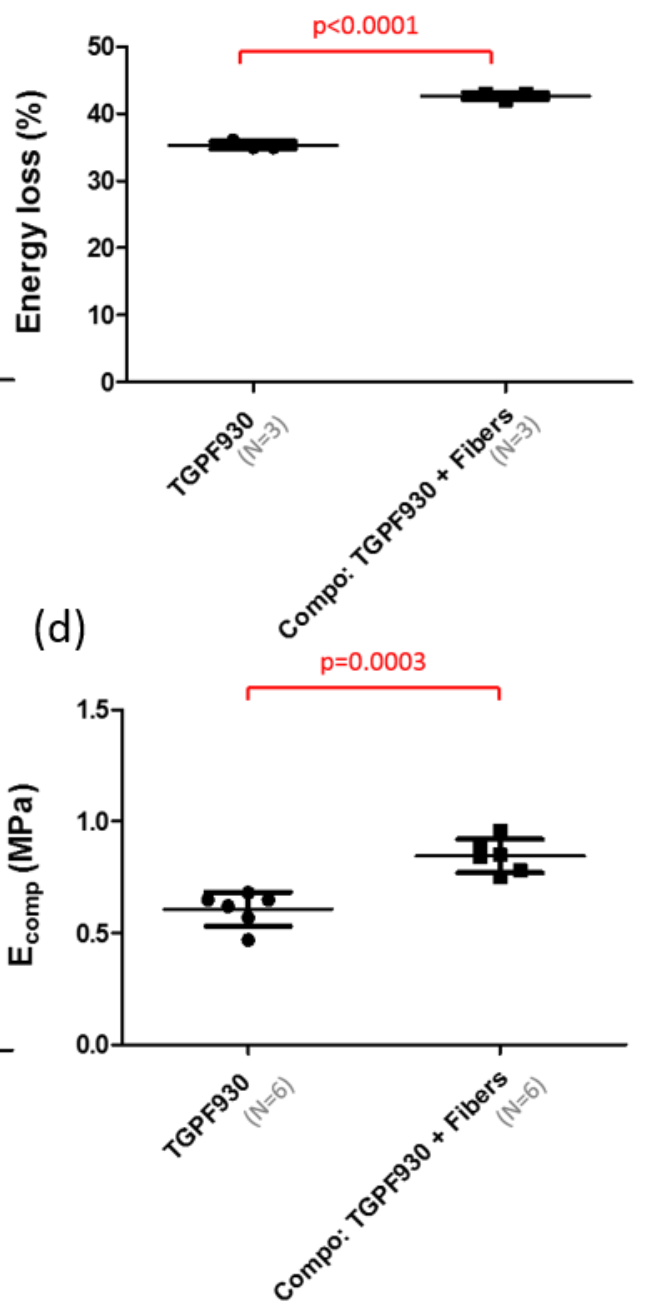

Figure 35. a) The apparent elastic modulus $\left(E_{\text {tensile(25\%) }}\right)$, b) energy loss, c) suture retention strength (SRS) and d) compressive modulus ( $E_{\text {comp }}$ ) of pure Tangoplus FullCure 930 (TGPF930) and two-material composite (Tangoplus FullCure 930 (TGPF930) with two layers of rigid Vero fibers). $p$ values of Unpaired t-tests.

\subsubsection{Control of the mechanical directional dependency of the 3D printed material}

To understand if fiber embedding could control the directional dependency of the material, one layer of two thick embedded Vero fibers (cross section area of $0.6 \times 0.8 \mathrm{~mm}^{2}$ ) was placed in the middle of a soft TGPF930 structure. The apparent elastic modulus in tensile of the axis at which the fibers were orientated was significantly higher (16\%) than the other one $(0.16 \pm 0.04 \mathrm{MPa}$ versus $0.13 \pm 0.03 \mathrm{MPa}, \mathrm{N}=8$ each, $p=0.0347$, Paired t-test) and but had minimal effect on the energy loss $(51 \pm 3 \%$ versus $57 \pm 8 \%, N=8$ each, $p=0.2032$ ) (Figure 36). Different in stiffness in each principal direction can therefore be obtained when fibers are embedded in the rubber-like material. 
(a)

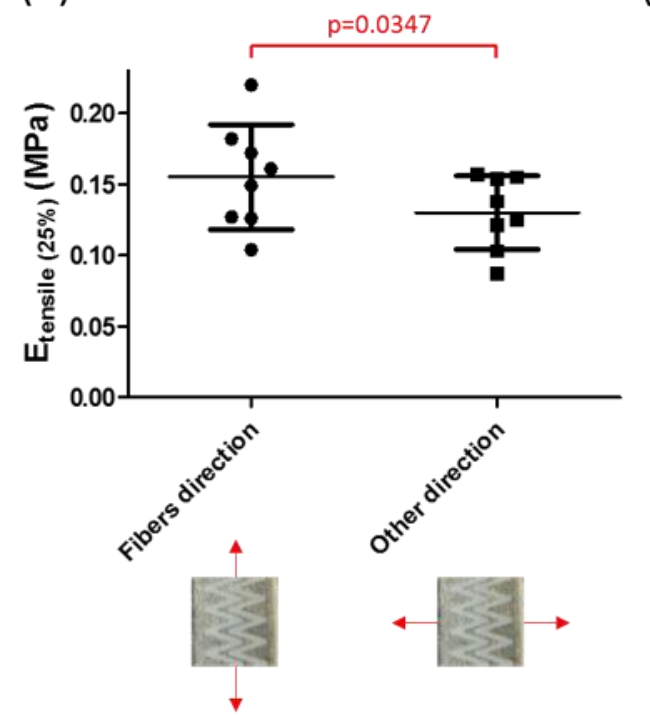

(b)

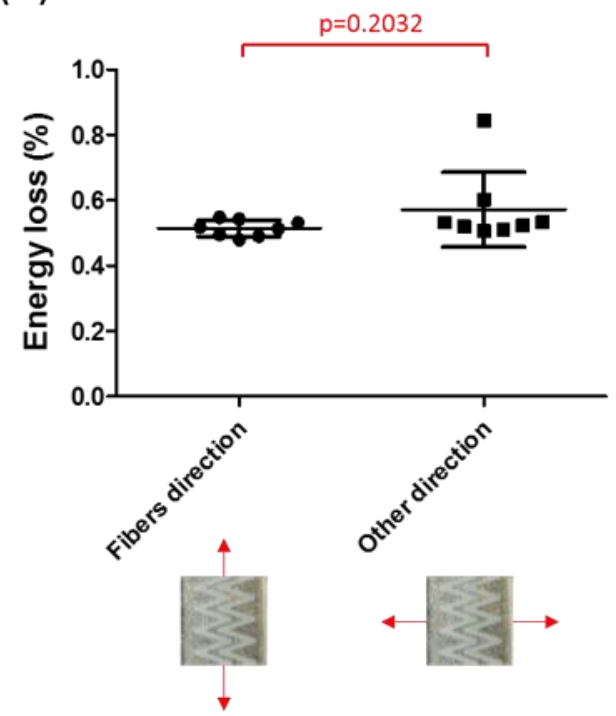

Figure 36. a) The apparent elastic modulus $\left(E_{\text {tensile(25\%) }}\right)$ and b) energy loss of the two principal directions of the two-material composite (Tangoplus FullCure (TGPF930) and one layer of large Vero fibers). p values of Paired t-tests.

\subsubsection{Tunable, soft but strong three-material composite}

\section{Comparison to the 3D printed material}

The above results were used to tune a three-material composite to create a synthetic aortic tissue. The results of a TGPF930 with two fiber layers (Vero White) material softened by support material (SUP705) are presented. There was not a significant difference of the three-material composite with pure TGPF930 (Unpaired t-test) in terms of apparent elastic modulus $(0.16 \pm 0.02 \mathrm{MPa}, \mathrm{N}=5, \mathrm{p}=0.6010)$ and SRS $(4.25 \pm 0.67 \mathrm{~N}, \mathrm{~N}=3, \mathrm{p}=0.6507)$ (Figure $37(\mathrm{a}-\mathrm{d})$ ). However, the energy loss $(40 \pm 3 \%, \mathrm{~N}=5, \mathrm{p}=0.0158$ ) was significantly higher and the compressive modulus $(0.39 \pm 0.03 \mathrm{MPa}, \mathrm{N}=6, \mathrm{p}<0.0001)$ lower. Consequently, adding support material (SUP705) softened the overall structure and increased visco-elasticity. The fibers allow the material to maintain integrity and allow for tuning of the material anisotropy.

\section{Comparison to the aortic tissue}

The SRS $(4.25 \pm 0.67 \mathrm{~N}, \mathrm{~N}=3, \mathrm{p}=0.6177)$ and compressive modulus $(0.39 \pm 0.03 \mathrm{MPa}, \mathrm{N}=6, \mathrm{p}=0.4485$ and $p=0.0596$ for normal and aneurysmal aortas respectively) of the three-material composite has shown similarities with the aortic tissue. The $25 \%$ strain apparent elastic modulus $(0.16 \pm 0.02 \mathrm{MPa}, \mathrm{N}=5, \mathrm{p}=0.0002$ and $p<0.0001$ for normal and aneurysmal aortas respectively) and energy loss $(39.92 \pm 2.27 \%, N=6$, $p<0.0001$ ) (Unpaired t-test) were still significantly stiffer and more viscous than human ATA (Figure 37 (eh)). 
When looking at the average loading curve of the three-material composite $(\mathrm{N}=6)$ in comparison to normal $(\mathrm{N}=3)$ and aneurysmal $(\mathrm{N}=4)$ aortic tissues in tensile testing at lower strains, the properties are very similar, Figure 38 . By $25 \%$, strain hardening of the composite occurs at a higher rate than the human tissue. 
Comparisons to the 3D printed material
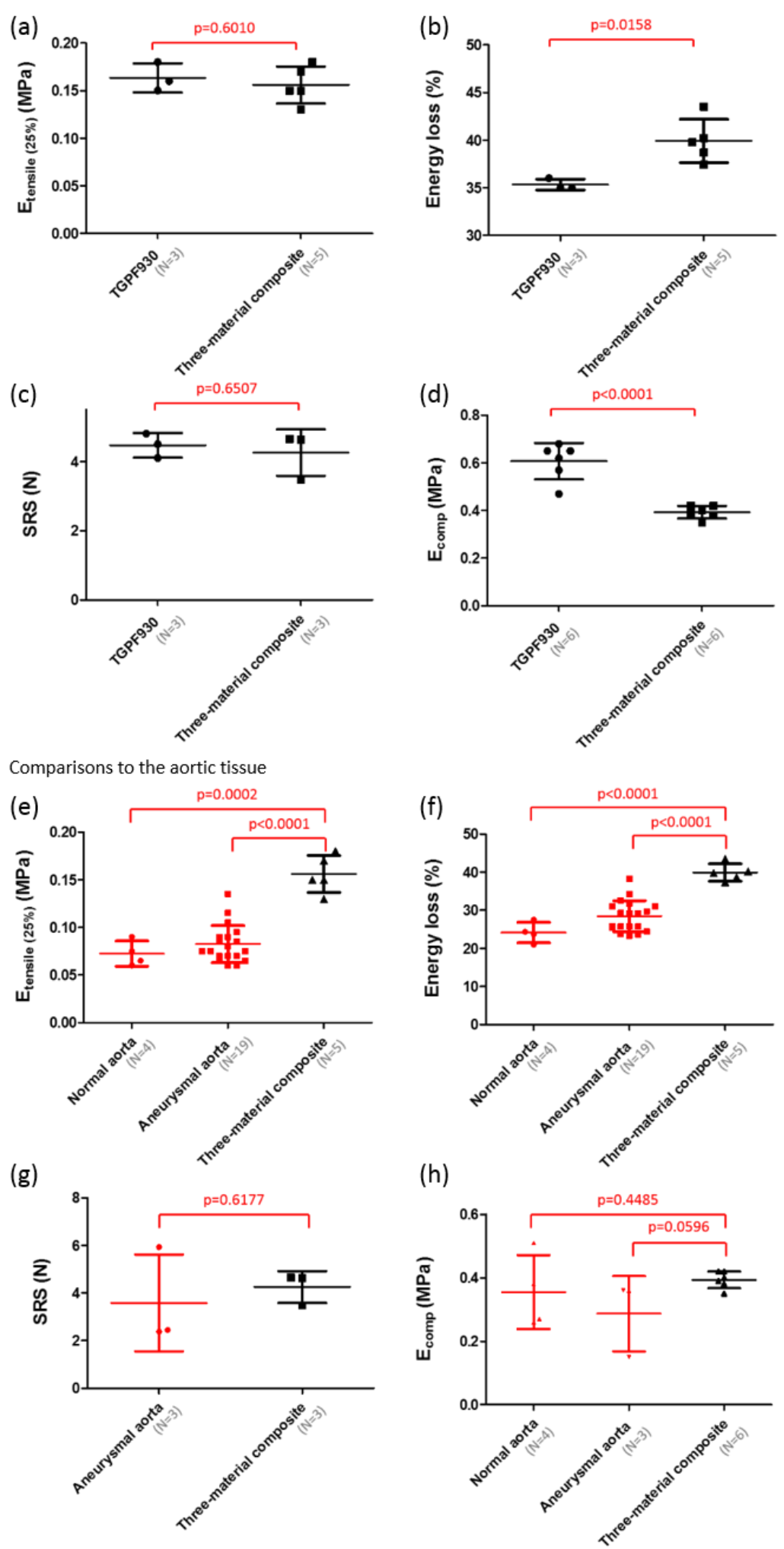

Figure 37. Comparisons of the three-material composite to the 3D printed composite (a-d) and aortic tissue (e-h) in terms of apparent elastic modulus $\left(E_{\text {tensile }(25 \%)}\right)(a, e)$, energy loss $(b, f)$, suture retention strength $(S R S)(c, f)$ and compressive modulus $\left(E_{\text {comp }}\right)(d, h) . p$ values of Unpaired t-tests. 


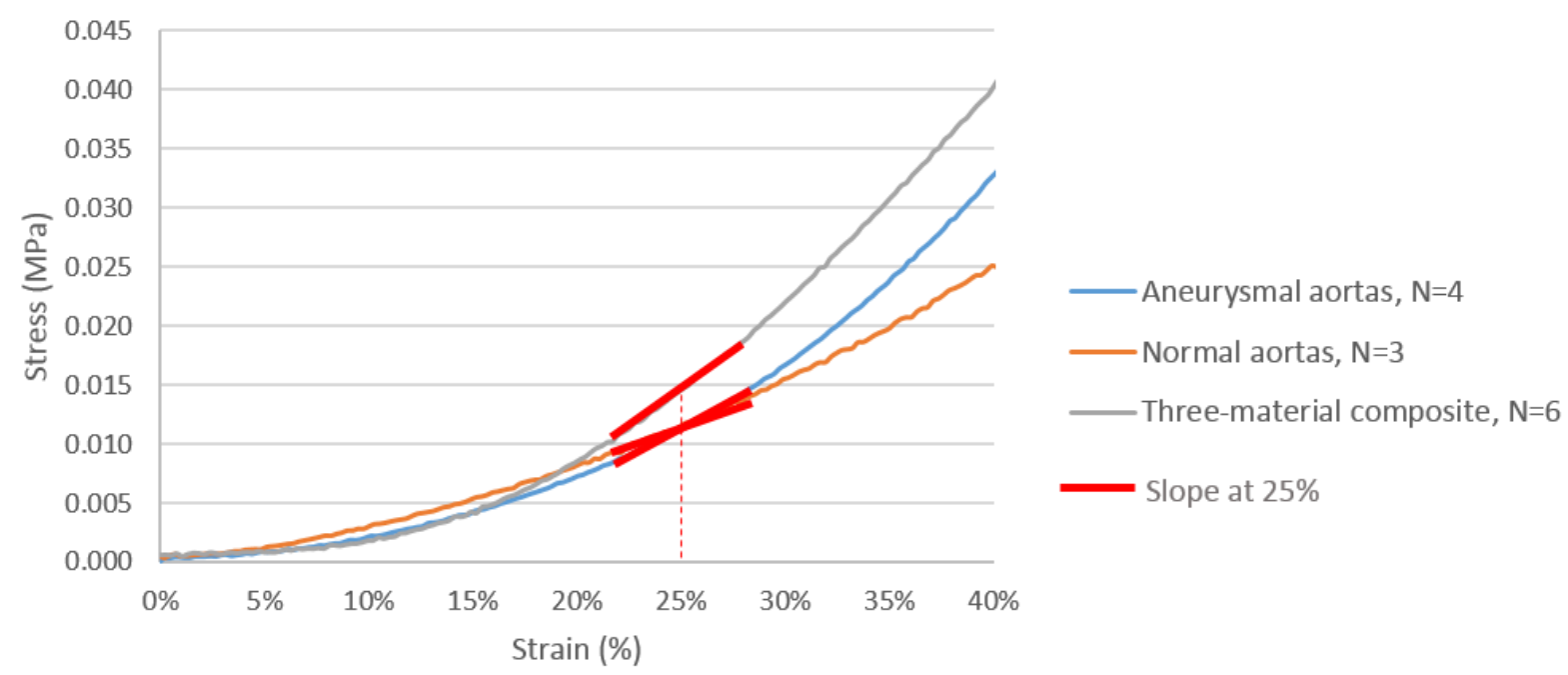

Figure 38. Stress and strain loading curves of normal and aneurysmal aortas, as well as three-material composite.

Table 5. Test applied and outcomes for each tested material.

\begin{tabular}{|c|c|c|}
\hline Material tested & Test applied & Outcome \\
\hline TGPF930 & $\begin{array}{l}\text { Equi-biaxial tensile test } \\
\text { Suture retention strength } \\
\text { Nano-indentation test }\end{array}$ & $\begin{array}{l}\text { Stiffer (in tensile testing) and more viscous than ATA } \\
\text { Strength similar to ATA }\end{array}$ \\
\hline $\begin{array}{l}\text { Compo: TGPF930 + } \\
\text { SUP705 }\end{array}$ & $\begin{array}{l}\text { Nano-indentation test } \\
\text { Suture retention strength }\end{array}$ & Softer and less resistance to suture tear than aortic tissue \\
\hline $\begin{array}{l}\text { Compo: TGPF930 + } \\
\text { Fibers (two layers) }\end{array}$ & $\begin{array}{l}\text { Equi-biaxial tensile test } \\
\text { Suture retention strength } \\
\text { Nano-indentation test }\end{array}$ & $\begin{array}{l}\text { Stiffer (in compression and tension) and better suture tear } \\
\text { resistance than TGPF930 }\end{array}$ \\
\hline $\begin{array}{l}\text { Compo: TGPF930 }+ \\
\text { One layer of thick } \\
\text { fibers }\end{array}$ & Equi-biaxial tensile test & Control of mechanical directional dependency \\
\hline $\begin{array}{l}\text { Three-material } \\
\text { composite }\end{array}$ & $\begin{array}{l}\text { Equi-biaxial tensile test } \\
\text { Suture retention strength } \\
\text { Nano-indentation test }\end{array}$ & $\begin{array}{l}\text { Less stiff (in tensile testing) and more viscous than Compo: } \\
\text { TGPF930 + Fibers (two layers) } \\
\text { Stiffer (in tensile testing) and more viscous than aortic tissues } \\
\text { Similar compressive stiffness and suture strength to ATA }\end{array}$ \\
\hline
\end{tabular}

\subsection{Discussion}

The 3D printed material TGPF930 has been used in surgical education and is suitable in some applications. However, the material properties of TGPF930 are uniform and isotropic. To resolve this issue, we created 3D printable composites using TGPF930 to create synthetic ATA tissue for surgical simulation. The mechanical testing was used to evaluate the tissue fidelity of our 3D printed models. The apparent elastic modulus and energy loss provide a measure of the dynamic response of the model due to the forces 
created by blood flow. The compressive modulus characterizes the feel or haptics and the suture retention strength simulates the material capacity of being sewed.

The study has shown how we can control the softness, strength, and mechanical directional dependency of the materials with a fibrous structure and the insertion of support material. However, there are limits to the mechanical properties that can be tuned with the currently available commercial materials. In the future, further fine-tuning can be done by varying the fiber geometries as well as the amount of filling in the soft structure. Despite the limitations, mechanical properties of the aortic tissue were replicated with the three-material composite such as the compressive stiffness and strength.

We found the tensile properties of TGPF930 stiffer and more viscous than ATA tissue but this may not be a fair comparison. The use of the same strain range for tensile testing assumes the samples start at a zerostress state. When ATA tissue is excised, it demonstrates significant residual stress and recoils when cut. It is known that the physiological strain range of the aorta during a cardiac cycle is in the range of 5-10\% due to blood pressure (Grøndal, Bramsen, Thomsen, Rasmussen, \& Lindholt, 2012; Morrison, Choi, Zarins, \& Taylor, 2009). To account for the residual stress in vivo, ex vivo biomechanical studies generally use higher strains (25\%, $40 \%$ or maximum apparent elastic modulus) to identify the stiffness of the tissue (Emmott et al., 2016). The 3D printed materials do not demonstrate significant residual stress when printed and would likely only see such high strains due to surgical manipulation. Moreover, small differences in stiffness and energy loss may not be perceptible for surgical training. Future studies should examine the physiological fidelity of the composites implemented in phantoms using pressure dilation testing, echocardiography imaging (Maragiannis et al., 2015), strain imaging (Petrini, Eriksson, Caidahl, \& Larsson, 2018) and subjective analysis of surgeons in training scenarios. In our study, we were able to reproduce the compressive modulus and SRS of ex vivo passive normal and aneurysmal ATA tissue. Matching these properties will help ensure the feel of manipulation and suturing are recreated in our synthetic models.

The novelty of our method is the ability to create heterogeneous and tunable composites using a commercial 3D Polyjet printer and materials. Despite the advancements in printing technology, commercial 3D printers do not allow the user to alter the polymer formulation, thus limiting the starting point for surgical simulation. Making combinations of materials is an alternative to controlling the polymer 
blend properties. To the best of our knowledge, this is the first study that has used the support material to soften the structure.

Our analyses showed that the mechanical behavior of the 3D printable composite is largely dominated by the properties of TGPF930 (apparent elastic modulus and energy loss). The fibers allow for adjusting the directional properties of the material while the support material softens the composite and increases its viscosity. Using 3D Polyjet printing, we can manipulate these properties to adjust local material properties necessary to recreate thoracic aortic pathologies.

Pathological ATA tissue is heterogeneous and can show a significant loss of anisotropy due to the degeneration and remodeling of the walls (Chung et al., 2017; Shahmansouri et al., 2016). Fibers of collagen of normal tissue are at a slight angle from the circumferential direction of the artery but tend towards the principal directions in distension state (Gasser, Ogden, \& Holzapfel, 2006). Those property variations between the principal directions of the tissue can be replicated with the use of fibers mimicking fibrous proteins like collagen with different angular orientations (Chen et al., 2011; Finlay, McCullough, \& Canham, 1995). Pathological tissue remodeling is also known to alter the ATA collagen and elastin structure resulting in large variations in material property amongst patients. This medical degeneration changes the anisotropy of the tissue (Chung et al., 2017; Shahmansouri et al., 2016). Therefore, adjusting these local properties could be key to tissue fidelity in aortopathies.

Others have used multi-material composites to alter mechanical properties of 3D printed models made with Polyjet technology. A recent study developed 3D printed microcells made of hard elliptical or ellipsoid like fibers (non-specified materials) randomly distributed in a soft TGPF930 matrix in order to improve micromechanical models used in finite element analyses for numerical simulations (Reiter \& Major, 2011). Materials were evaluated in tensile and compressive testing but were not compared to biological tissues. Similarly, K. Wang, Wu, et al. (2016) combined soft (TGPF930) and rigid Vero materials for soft patientspecific tissue-mimicking phantoms but neglected the mechanical directional dependency of the overall structure by using fibers in only one direction. They also limited the assessment of their 3D printing multimaterials to uniaxial testing only, which may not be enough for a proper validation and representation of potentially anisotropic biological tissues. In addition, Vukicevic et al. [6] mixed both materials (TGPF930 and Vero) during the printing in order to change the hardness of the model. Two blends were created to differentiate the external structure and inner core of the valve leaflets in a model used for mitral valve repair strategies. However, both resulting materials remained homogeneous and 
isotropic, which could potentially be a major limitation for a proper representation of soft tissue, as mentioned above.

We have implemented the three-material composite in a patient-specific aortic geometry obtained by imaging data with the method developed by Garcia et al. (2017). To further our development, the 3D patient specific aortic models with high tissue fidelity need to be evaluated by experienced surgeons to validate the best combinations for task specific vascular surgical training.

Beyond aortic surgery, the tunable 3D printed material could be used for other applications. Alterations of the composite structure would potentially allow accurate representations of other soft tissues and whole organs. These phantoms will also be very useful for evaluating medical devices and imaging techniques.

\subsection{Conclusion}

In this study we reported that mechanical properties of 3D printed composites (hardness, apparent elastic modulus, energy loss, compressive modulus and SRS) can be controlled and adjusted by using rigid fibers and brittle support material. We replicated the ex vivo passive tissue characteristics of aortas, and therefore were able to improve the current homogeneous 3D printed TGPF930 material often used in surgical education. 


\section{Chapter 6: Paper 3}

The manuscript is in preparation for submission to Journal of Surgical Simulation.

The paper deals with the creation of dynamic and echo-compatible aortic simulators with geometric and physiological fidelity. It builds on the previous paper (Garcia et al., 2018) which presented composite materials to mimic aortic tissue collected from patients. One of the structures of that study was taken and implemented to different geometries and tested by echocardiography. Appendix 5 explained how to implement the composite structure in the aortic geometries to obtain the phantoms.

The aortic model material properties were analyzed by mechanical testing and in a simulated in vivo environment by incorporating it into a mock circulatory system for speckle tracking echocardiography (SPE) measurements. SPE is a technique that quantifies the wall movement by tracking natural acoustic reflections of cross-sectional areas (physiological fidelity Objective 2.3). The perfused system would also allow the practice of entire surgeries instead of limiting the practice to simple and individual tasks (Objective 3). The study showed that the simulated in vivo measures co-varied with the destructive mechanical testing. Moreover, we found that that only the material composition had an impact on the overall mechanics of the models and the dynamic echo-compatible aortic simulators were able to reproduce motions and properties of real aortas.

To conclude, we developed an enhanced and realistic aortic simulator for the practice of entire surgeries. 


\title{
3D printed ascending aortic simulators with physiological fidelity for surgical
}

\author{
simulation
}

Justine Garcia ${ }^{1}$, Ali Alakhtar ${ }^{2}$, Mansour AlOmran ${ }^{2}$, Cornelius Hart ${ }^{1}$, Alexander Emmott ${ }^{3}$, Rosaire Mongrain ${ }^{1}$, Richard L. Leask ${ }^{3}$, Kevin Lachapelle ${ }^{2 *}$

${ }^{(1)}$ Mechanical Engineering, McGill University, Montreal, Quebec, Canada

(2) Cardiovascular Surgery, McGill University Health Network, Montreal, Quebec, Canada

${ }^{(3)}$ Chemical Engineering, McGill University, Montreal, Quebec, Canada

* corresponding author

Keywords: Aortic simulator, Physiological fidelity, Speckle tracking, Echocardiography

\subsection{Abstract}

Surgical simulation is an essential part of training for cardiac surgery. It helps trainees to develop skills, knowledge and experience. Nevertheless, the current aortic simulators remain very simplistic and can not replicate the variety of geometries or material properties of diseased aortas. For these reasons, we developed a novel technique to create 3D printed aortic models to be able to fabricate unique aortic geometries and adapt the material properties to better represent patient data. In this study, we show the effect of geometry and material properties on the physiological fidelity of the models.

Models were 3D printed with a Connex3 Objet500 3D printer (Stratasys, Eden Prairie, USA) with two geometries and two different material compositions. A set of straight tube models and a set of idealized ascending aortic aneurysm models where produced. For each geometry a one material elastic model made of TGPF930 (Tangoplus FullCure 930, Stratasys, Eden Prairie, USA) was printed. A second set of models was designed to replicate the natural structure of the aorta by imitating the collagen with embedded solid zigzag fibers made of Verowhite (Stratasys, Eden Prairie, USA) in a flexible TGPF930 structure meant to simulate the elastin. 
Each model was evaluated by ex vivo tensile testing (apparent elastic modulus and energy loss) and in vitro dynamic speckle tracking echocardiography SPE (cardiac cycle pressure modulus (CCPM)) to investigate the role of material composition/geometry on the physiological fidelity. The speckle track echocardiography was compared to our patient cohort data ( $N=5$, Age: $77 \pm 6$ years old).

Two-way Anova analyses showed that the ex vivo material properties were only dependent on model composition (apparent elastic modulus: $p \leq 0.01$ and energy loss: $p \leq 0.001, N=3$ for each category of simulators), but not the geometry. Similarly, SPE analysis showed the model composition, not the geometry determines the physiological fidelity. There was good covariance between the apparent elastic modulus at $25 \%$ strains and the energy loss obtained by tensile testing versus CCPM in SPE (apparent elastic modulus: $r^{2}=0.5579, p=0.0041, N=12$ and energy loss: $r^{2}=0.8173, p<0.0001, N=12$ ). Finally, when comparing the SPE data ( $\mathrm{N}=3$ for each category of simulators) with patient data $(\mathrm{N}=5)$, we showed that TGPF930 tubes and aortas were able to replicate the apparent vessel wall deformations of real in vivo data. Moreover, adding fibers to the models had the ability to mimic the anisotropy and stiffest aortic tissue properties which could not be replicated by single material TGP930 models.

The results provide a means to create surgical training models of the ascending aorta with biomechanical physiological fidelity of patient specific geometry which will help advance surgical training.

\subsection{Introduction}

Surgeons require a high skill set that is traditionally acquired through an intensive training in the clinic which, for several years now, includes some form of medical simulation. This educational method allows for repeated practice at any time in a safe and controlled environments without harming patients (de Montbrun \& MacRae, 2012; Dutta \& Krummel, 2006). This improves knowledge, confidence as well as management skills of the trainees (Chakravarthy et al., 2011).

Ideal cardiac surgical simulations involve realistic cardiovascular systems. However, the current vascular simulators remained oversimplified with no perfusion and basic tubular shapes for complex aortic or venous geometries (Cao, Duhamel, Olympe, Ramond, \& Langevin, 2013; Pazos et al., 2010; Pepley et al., 2018; Sulaiman et al., 2008). The creation of patient-specific models with tuned material properties is possible from 3D printing (Garcia et al., 2017). These methods can create models that have geometric and material property fidelity suitable for surgical training involving the ascending aorta. 
To provide optimal surgical training, models also need to have biomechanical physiological fidelity. The model vessel should be able to replicate the tissue deformations created by the dynamics of blood flow. Ideally, surgical simulators would be perfused and provide realistic pulse pressure-diameter fidelity for a variety of pathological states to allow trainees to practice surgical skills on moving vessels. For example, ascending thoracic aneurysmal aortas (ATAA) can demonstrate aberrant hemodynamics and tissue distension when compared to a healthy ATA. These differences are created by abnormal left ventricle outflow tack blood flow and pathological tissue remodeling characterized by elastin degradation and loss of medial structure resulting in a dilation of the vessel (Chung et al., 2017; Holzapfel et al., 2004; Iliopoulos et al., 2009; Mulvany \& Aalkjaer, 1990; Shahmansouri et al., 2016). To the best of our knowledge, no study has shown biomechanical physiological fidelity in a surgical simulator of the ascending aorta.

The creation of a new generation of dynamic aortic simulators suitable for mock flow circulation will enhance the current cardiac surgical training. We developed 3D printing methods that allow for the creation of models with geometric fidelity from patient images (Garcia et al., 2017), as well as multimaterial structure able to adapt local mechanical properties and mimic human aortic tissue (Garcia et al., 2018). In this study, we evaluated the capacity of the aortic simulators to replicate the deformations of real aortic tissue (physiological/ tissue fidelity) by speckle tracking echocardiography (Geyer et al., 2010). In addition, we investigated if the physiological fidelity of the models is dependent on material and/or geometry.

\subsection{Materials and methods}

\subsubsection{Geometries and materials of the 3D printed aortic simulators}

Aortic models were developed by computer-aided design with a combination of two software (Solidworks (Dassault Systèmes, Waltham, USA), Rhinoceros 3D (Robert McNeel \& Associates, Seattle, USA)), and then 3D printed with a Connex3 Objet500 printer (Stratasys, Eden Prairie, USA). To understand the role of material composition and geometry on the physiological fidelity, two geometries were created using two different material compositions (Figure 39). Similar to our previous work (Garcia et al., 2018), we used a Connex3 Objet500 to 3D print our models, which were made of materials that have proven to be echogenic (Maragiannis et al., 2015). The physiological fidelity was assessed by speckle tracking echocardiography (SPE). 
Straight tube models and an idealized ATAA geometry were created with and without inlaid fibers. The single-material models were made with the homogeneous, isotropic and elastic TGPF930 only (Tangoplus FullCure 930, Stratasys, Eden Prairie, USA). This 3D printed resin has already been used in the literature, however the use of this material has limitations as it does not have the ability to tune any mechanical properties. The two-material composite (Figure 39) was designed to broadly replicate the natural structure of the aortic tissue by imitating the mechanics and geometry of the fibrils of collagen. The latter were represented by zigzag fibers made of Verowhite (Stratasys, Eden Prairie, USA) embedded in the flexible TGPF930 material to simulate the elastin lamellae. The structure has shown its ability to control the softness (feel or haptic), stiffness (resistance of deformation) and mechanical directional dependency (anisotropy) of the material (Garcia et al., 2018).

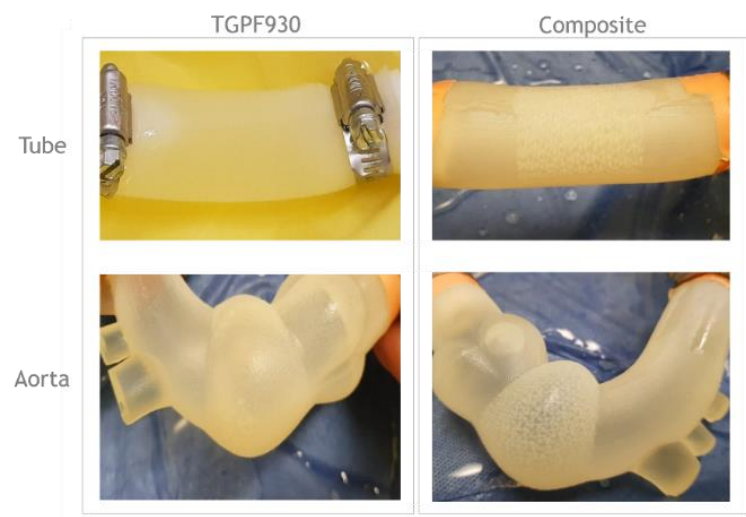

Figure 39 Aortic simulators with the two geometries (tube, aorta or idealized ATAA) and material (TGPF930, Composite).

\subsubsection{Echocardiography and dynamic speckle tracking}

A GE Vivid E95 echocardiographic unit (GE Healthcare, Chicago, USA) was used to obtain the imaging data for all analyses (patients and aortic simulators). By placing Cardiac probe (M5Sc) on the anterior surface of the model, we acquire short axis images under pulsatile flow loop with a mean pressure of $90 \mathrm{mmHg}$. The settings of the echocardiographic machine are as the following: frequency $2.3 / 4.6 \mathrm{MHz}$, AutoGain 2 $\mathrm{dB}$, compress $60 \mathrm{~dB}$ and depth $8.0 \mathrm{~cm}$. 


\section{Aortic simulators}

Perfusion studies of the models were performed within 7 days of printing. A pulsatile flow loop consisting of a positive displacement piston pump (Cardioflow MR 1000, Shelley Medical, Toronto, Canada), pressure sensor (86A, TE Connectivity Measurement Specialties), resistance valve and an aortic model mounted in a water-bath as shown in Figure 40. For each SPE acquisition, a $0.5 \mathrm{~Hz}$ sinusoidal flow waveform was created by the pump and the relative diameter was measured (Figure 40). The pressure in the flow loop was adjusted to a mean pressure of $90 \mathrm{mmHg}$. The pressure and STE data was collected for 3 cycles and averaged to ensure a nontruncated cycle.

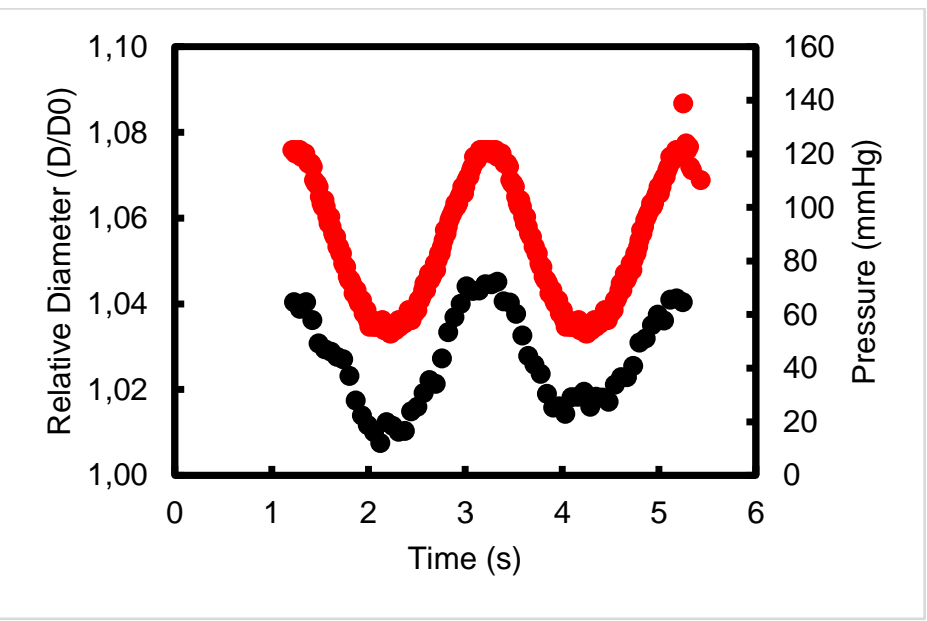

Figure 40. Relative diameters and corresponding pressure obtained for the perfusion system

Therefore, the data simultaneously acquired during the experiments were (1) the instantaneous crosssectional areas (thus deformations) of the simulators at the middle of the tube or idealized ATA aneurysm and (2) the simulated blood pressure obtained by the programmable piston-pump. 
a)

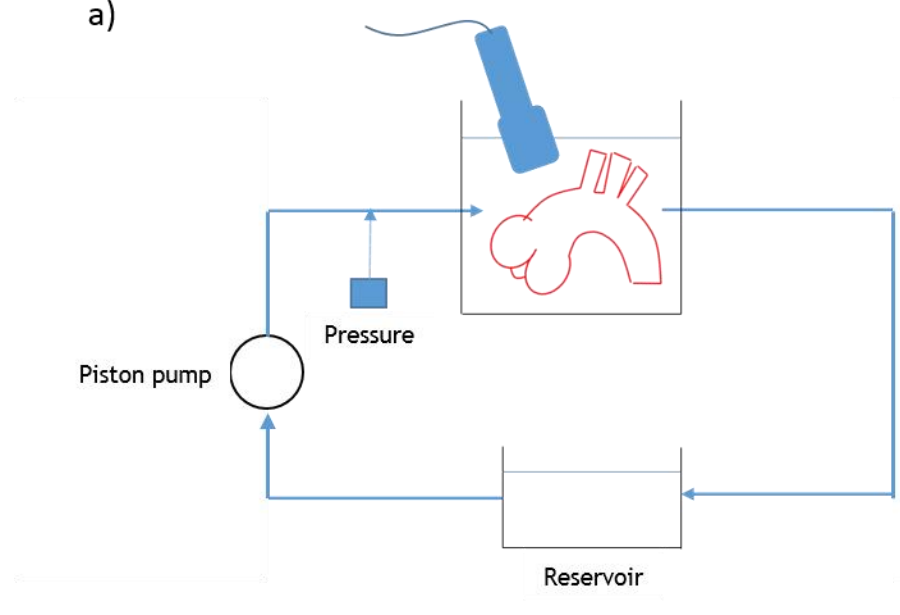

b)

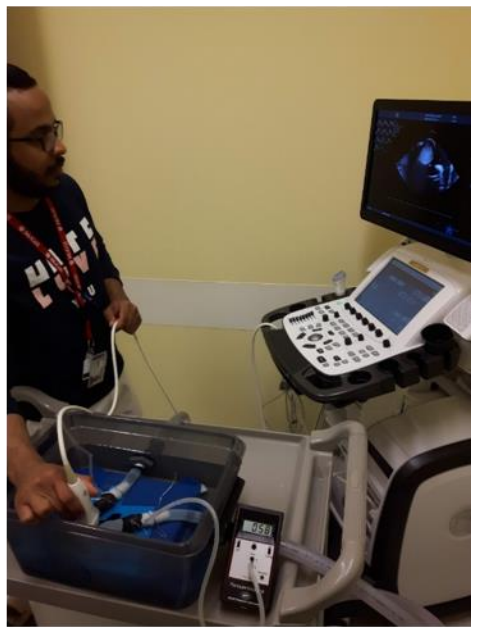

Figure 41. a) Piston-pump system for the evaluation of the aortic simulator by SPE and b) Picture of the operator performing the evaluation.

\section{Patient population}

Informed consents were obtained from patients in compliance with the Canadian tri-council policy statement on ethical conduct for research involving humans (Emmott et al., 2018). Patients were administered anesthetic before we recorded the aortic expansions with a transesophageal echocardiography probe inserted into the esophagus to record the maximum deformations of three cardiac cycles, as explained in a previous study led by Emmott et al. (2018) in our lab. The following data were recorded for each patient: (1) the instantaneous aortic cross-sectional areas (thus deformations), (2) the electrocardiogram and (3) the calibrated pulse tracing of 5 patients (Age : $77 \pm 6$ years old).

\section{Speckle tracking analyses of both sets of data}

Analyses were performed with short-axis view and processed with GE's Echo-PAC software (GE Healthcare, Chicago, IL, USA). The 2D strain analysis (SAX-PM for the model) function was used to manually place markers (or speckles) on the instantaneous lumen areas (obtained for patients or simulators) to track the displacements over time (Figure 42). From the displacements were finally computed the circumferential stretches ( $\left.\lambda_{\text {circ }}\right)$ of the aortas, as well as the maximum diameters.

Pressure-stretch curves were then obtained for every sample and patient aortas and hysteresis loops were obtained. Slopes of the linear fits of the pressure and circumferential stretch $\left(\lambda_{\text {circ }}\right)$ curves were calculated to define the cardiac cycle pressure modulus (CCPM, slope of the curve) (Figure 42), which was originally 
developed to better predict the need of surgery than the use of the aortic diameter (Emmott et al., 2018). Moreover, we took the ex vivo tissue thicknesses for the in vivo studies.

a)

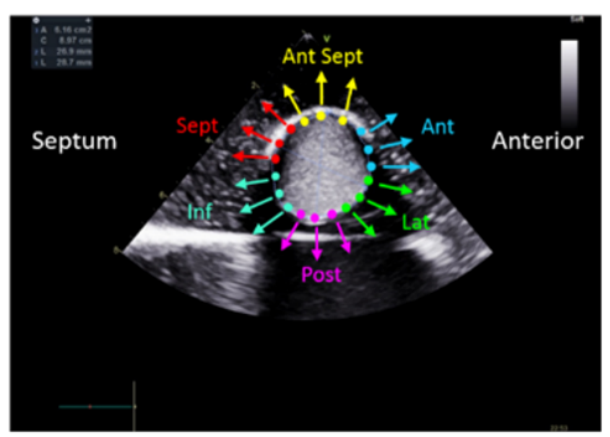

b)

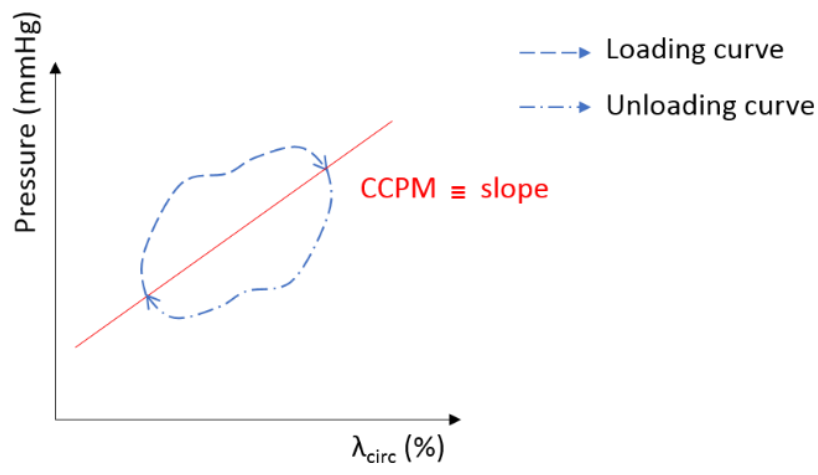

Figure 42. a) Points taken along the aortic walls track displacements over time for the septal (sept), anteroseptal (ant sept), anterior (ant), lateral (lat), posterior (post) and interior (int) segments. b) Pressure-circumferential stretch curve to determine the slope, thus the Cardiac cycle pressure modulus (CCPM).

\subsubsection{Equi-biaxial tensile testing}

Equi-biaxial tensile testing is a common method to evaluate ex vivo material properties of the aortic tissue (Emmott et al., 2016). In this study, we used this method to compare the simulator outputs obtained by ex vivo (short squared samples extracted from the simulators) and in vivo (entire aortic simulators) testing. If we obtain a good covariance between the methods, it would mean that findings obtained by tensile testing could therefore be used to predict echo testing.

All simulators were tested after SPE testing and within a week after fabrication. The same operators did the testing with the same equipment. We have a limited number of samples, but the distribution is assumed to be normal. Informed consents were obtained from patients in compliance with the Canadian tri-council policy statement on ethical conduct for research involving humans.

Samples taken from patient aortas were collected at the time of surgery and stored at $4^{\circ} \mathrm{C}$ in a saline solution before carrying out the testing within 24 hours of excision. Normal tissue was obtained from donors. 
Squared samples $\left(15 \times 15 \mathrm{~mm}^{2}\right)$ were stretched in a bath $\left(37^{\circ} \mathrm{c}\right.$ saline solution for the tissue and room temperature water for the 3D printed models) with a constant strain rate $(0.1 \mathrm{~mm} / \mathrm{s})$ and silk sutures connected to the ElectroForce ${ }^{\circledR}$ Planar Biaxial TestBench tensile tester (TA Instruments, New Castle, DE, USA) (Figure 43, a). The two-parameter Mooney-Rivlin energy function was used to fit the loading stressstrain curves and calculate the apparent elastic modulus (or slope of the loading curve also-known-as stiffness $E_{\text {tensile } 25 \%}$ ) at $25 \%$ strains, as well as the energy loss (Figure $43, b$ ). The energy loss is quantifying the energy that would be absorbed and returned by the material, as does an aorta under physiological function during heart cycles (Chung et al., 2014)

a)

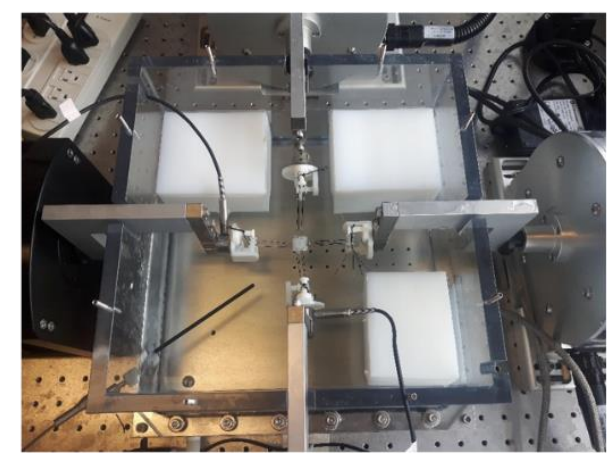

b)

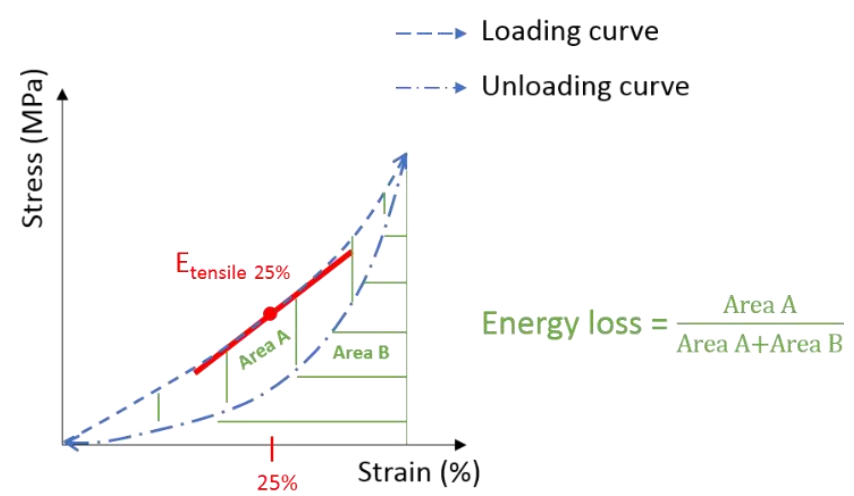

Figure 43. a) Equi-biaxial tensile tester with the generated stress-strain curves obtained from the stretching and b) the Apparent elastic modulus $E_{\text {tensile } 25 \%}$ as well as the Energy loss were calculated with the slope of the loading curve at $25 \%$ strains.

\subsubsection{Statistical analysis}

Data are presented as mean \pm standard deviation and the analyses were performed by using GraphPad Prism 5 (GraphPad Software, Inc., La Jolla, USA). The significance was achieved at $p$ values $\leq 0.05$. The analyses used in the study started with the linear regression to determine the covariance. Covariance plots are presented with a linear regression line (solid, black) and a 95\% confidence interval (dotted, black). In addition, we used a Two-way Anova and One-way Anova with Tukey's multiple comparison test to compare sets of data.

\subsection{Results}

A total of 3 models per geometries and materials were 3D printed for the study and the experiments were carried out within a week after their creations. 


\subsubsection{Effects of the geometry and material on the model tensile properties}

Figure 44 displays the average and standard deviation of the tensile testing results for 3 replicate models in each category. Two-way Anova analyses confirms that the tensile properties of the models were dependent on the material composition but not the geometry ( $E_{\text {tensile } 25 \%} p \leq 0.01$ and energy loss $p \leq 0.001$ respectively, $\mathrm{N}=3$ for each category of simulators).

a)

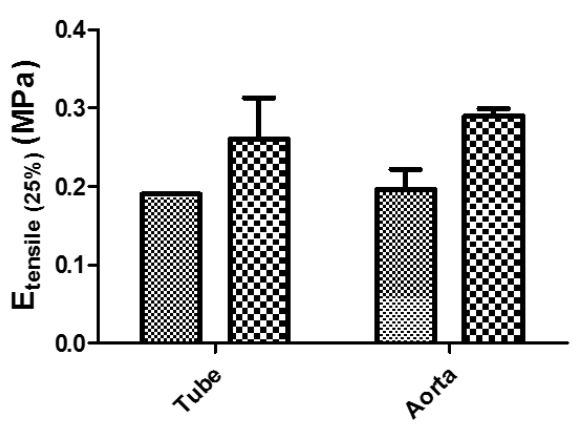

b)

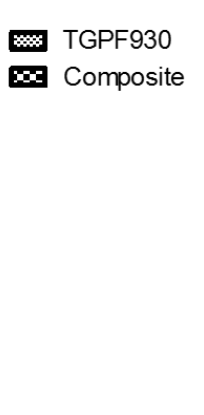

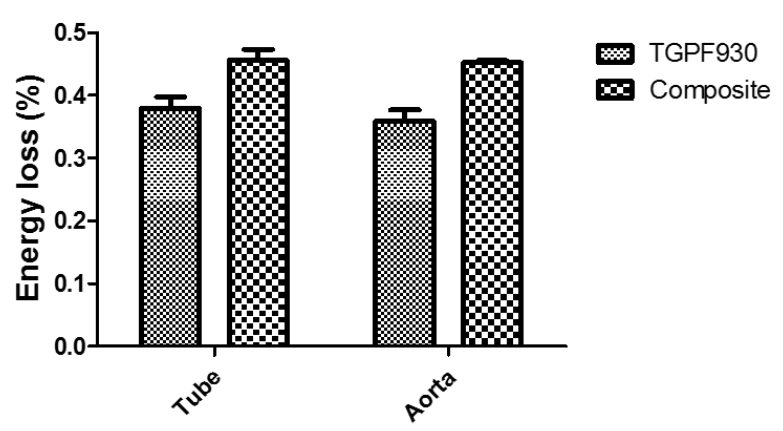

Figure 44. Two-way Anova analyses to compare the effects of geometries and materials to the simulators with the a) Apparent elastic modulus ( $\left.E_{\text {tensile } 25 \%}\right)$ and $b$ ) Energy loss.

\subsubsection{Comparison of SPE to tensile testing}

TGPF930 already proved its echogenicity (Maragiannis et al., 2015), but the analyses showed that the composite was also echo-compatible for SPE analysis. The CCPM was compared to the apparent elastic modulus at $25 \%$ strains ( $E_{\text {tensile } 25 \%}$ ) and energy loss obtained by tensile testing (well-known method for material evaluation). We found that CCPM had a strong significant covariance with the apparent elastic modulus $E_{\text {tensile } 25 \%}\left(r^{2}=0.5579, p=0.0041, N=12\right)$ (Figure 45) and energy loss $\left(r^{2}=0.8173, p<0.0001 \mathrm{~N}=12\right)$. (Figure 45). Similar correlations were found between the ex vivo parameters and patient data (Emmott et al., 2018). 
a)

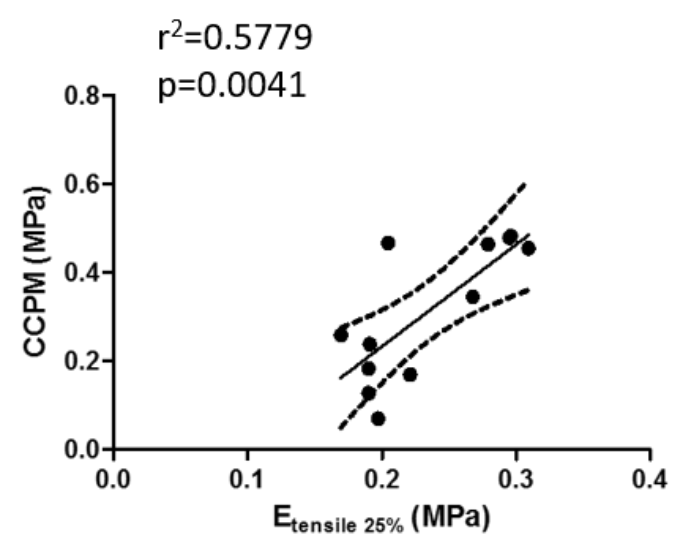

b)

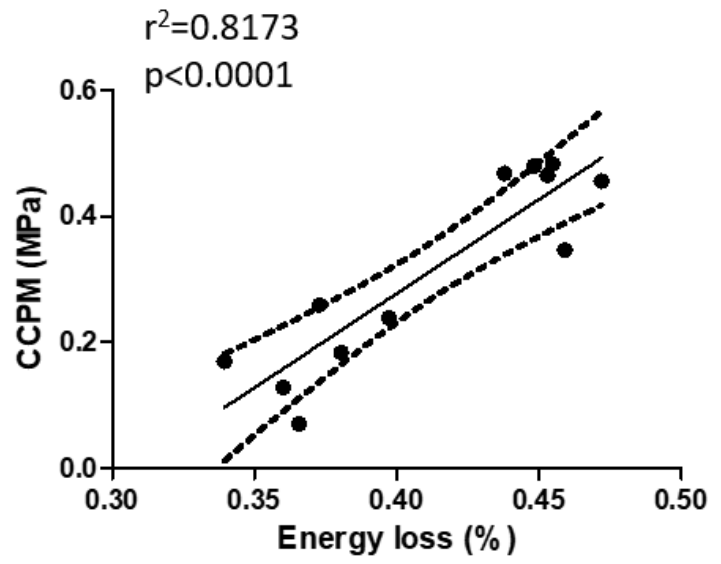

Figure 45. Comparison by linear regression of CCPM (Cycle Pressure Modulus) to the a) Apparent elastic modulus ( $\left.E_{\text {tensile } 25 \%}\right)$ and b) Energy loss.

\subsubsection{Comparison with aortic tissue}

We had a total of 5 patients for the study taken from a study previously led in our lab (Emmott et al., 2018). The One-way Anova analyses and Tukey's multiple comparison tests were used to compare the aortic simulators to in vivo patient data with CCPM. Similarities were found between TGPF930 models (tube and ATAA, $\mathrm{N}=3$ for each type of simulators) and real aortas $(\mathrm{N}=5)$. Moreover, Figure 46 shows the ability of the composite to mimic the hardest tissues obtained from the patients.

When the composite was used, we found higher CCPM than for models made of TGPF930 which proves than using fibers allow for tuning the properties of the aortic walls. However, the amount of fibers that was used in this study was probably too high, and their widths too large. Therefore, we will need to reduce both in order to mimic more patient aortic tissue. Being able to produce elastic material with controllable stiffness is key as it could be used to make more realistic 3D printed aortic tissue with local hotspots.

Taken together the results show 3D printed dynamic aortic simulators can replicate local deformations of patient aortas tested by SPE. TGPF930 allows for similar deformations to human tissue, however pathological tissue could show high stiffness that this homogeneous resin can not reproduce. Therefore, we can see that using a 3D printed composite has the ability to change properties of the synthetic walls, thus would be able to mimic a larger population of patients which characteristics are known to show very large variations. 


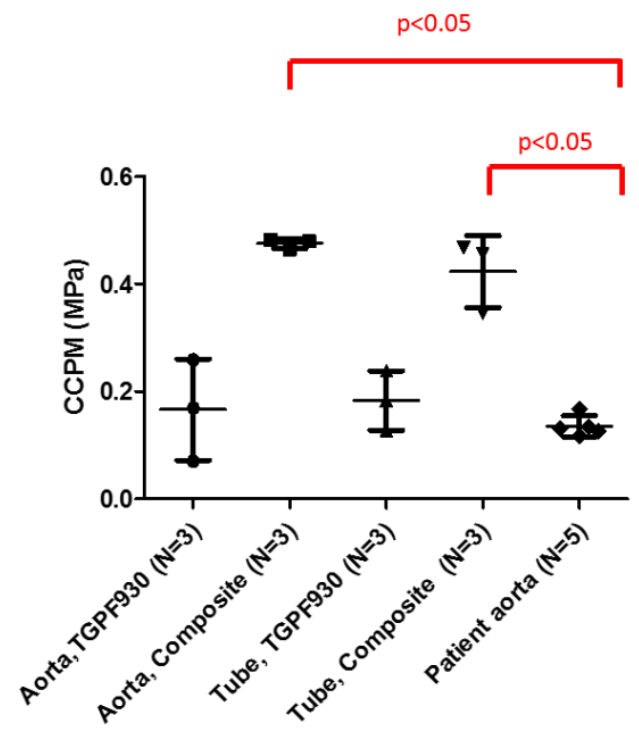

Figure 46.One-way Anova and Tukey's multiple comparison tests to compare the 3D printed models to patient aortas.

\subsection{Discussion}

In this study we demonstrated 3D printed models can replicate the physiological deformation of a human ascending aortic aneurysm. This biomechanical physiological fidelity is necessary to improve the look and feel of aortopathy surgical simulation training. Furthermore, we showed SPE could be used on the models to estimate the material properties and that these estimates were representative of ex vivo tensile testing (apparent elastic modulus and energy loss).

The study shows that the geometry of the 3D printed model has little effect on the physiological distension. Physiological fidelity can therefore be achieved by altering the material composition and not be affected if complex geometries, as captured patient specific aortas, are replicated. This fidelity is essential for a good simulated surgical training to prepare surgeons to real situations and apprehend the tactile feel of the tissue - important for skill developments (Lujan \& DiCarlo, 2006; Shah et al., 2017). Moreover, it allows the dynamic models to move with simulated heart cycles when such motions are key for the practice of entire surgical procedures. The replication of realistic parts of the cardiovascular system for complete operations remained unfortunately inexistent on the market, thus needed to be developed.

The possibility of tuning synthetic tissue that could be implemented in any geometrical simulators would inevitably enhance the current training models on the market. As shown in the literature, properties of 
the aortic tissue remain very complex and would vary within the population. There is therefore a need to be able to control the mechanics of the simulator walls to change characteristics in accordance to pathologies and disease stages (Chung et al., 2014; Pisano et al., 2011; Schlatmann \& Becker, 1977), instead of using single and not tunable materials for arteries, (Biglino et al., 2013; Kurenov et al., 2015), mitral valves (Vukicevic et al., 2016) or cerebral aneurysms (Wurm et al., 2011) Studies demonstrated that pathological aortic tissue would have stiffness increases (Iliopoulos et al., 2009) resulting in an isotropic response of the tissue (Chung et al., 2017; Shahmansouri et al., 2016) instead of anisotropic for normal aortas (Holzapfel et al., 2004; Mulvany \& Aalkjaer, 1990). Those are consequences of tissue remodeling which is degrading fibrils of elastin controlling passive elastic behavior when collagen is in charge of tensile strength (Tsamis et al., 2013). They are both largely present in the adventitia and media layers for normal tissue.

The solution that we developed was the creation of 3D printed composites that could be indefinitely tuned to imitate a wide variety of patient aortic tissue by controlling the softness (feel or haptic), strength (resistance of deformation) and mechanical directional dependency of the material (Garcia et al., 2018). In this study, we found that the use of our fibers gave a high CCPM stiffness, thus we would need to reduce their amount and/or size to better mimic the aortic tissue. However, it proved that using fibers controls mechanical properties of the 3D printed materials and would therefore allow the possibility to 3D print higher fidelity models with local structure variations, thus replications of local mechanical response.

The main limitation of the study remained in the limited numbers of samples. Properties of 3D printed models were assumed very consistent, however we were limited to 5 patient samples for SPE which may not provide a good representation of a diverse patient population. One of the reasons is that echocardiography used for aortic tissue evaluations is still a new method which was originally used for initial diagnoses to assess the global motion of the vessels in response to the stresses caused by blood pressure. Today, researchers are developing the technique to help medical professionals make surgical decisions (Satriano, Rivolo, Martufi, Finol, \& Di Martino, 2015) as it is known that distensibility of the aortic tissue was correlating with risks of rupture. The use of echo-parameters could therefore replace or complete the current predictor of dangerous conditions (Di Martino et al., 2006; Wilson et al., 2003).

For future work, we would need to determine ex vivo and/or in vivo mechanical properties of a large combination of composites (with different fiber densities, widths, patterns for instance), in order to adapt 
the structures to patient specific aortas. A larger collection of patient data would also be required to have a better representation of the patient population properties. Those additional analyses would allow us to create even more realistic simulators with local mechanical variations to mimic the exact tissue mechanical responses. In addition, we would need to test the circuit with more realistic blood-mimicking liquid to enhance the training and validate the models by medical experts.

\subsection{Conclusion}

In the study, we developed dynamic echo-compatible aortic models able to reproduce motions thus properties of real aortas (physiological fidelity). It was found that ex vivo results obtained by tensile testing could be predicted from in vivo SPE analyses. The study has also shown that only the materials would impact the overall mechanics of the models. Those aortic surgical simulators would therefore enhance simulation training, ideal the practice of an entire surgery. 


\section{Chapter 7: Discussion and Conclusions}

Medical simulation is fundamental for the education of the future generations of physicians and surgeons. Strong knowledge and motor skills would be gained during classes providing learning activities in the form of practical exercises with specific-task training tools and/or simulated environments. In the thesis, I focused the research on the physical (thus not virtual) simulation of the ATA. Cardiac simulators currently used by simulation centres remain very simplistic with basic tubular geometries and/or may not necessarily have representative material properties. For these reasons, I investigated whether 3D printed composites could be used to represent human aortic tissue needed for physical surgical simulation. Therefore, the objective of this research was to produce synthetic 3D printed models with specific anatomical similarities (anatomic fidelity) and biomechanical properties of both normal and pathologic human aortas (tissue fidelity). For this purpose, we selected the high resolution (20-30 microns) Polyjet technology known for its fast building speed and ability to create multi-material objects with a wide range of coloured materials with specific properties (Biglino et al., 2013; Cloonan et al., 2014; Oxman et al., 2012; Reiter \& Major, 2011). This UV curing method is expensive (high machine and material costs) but remains today the only one with sufficient versatility.

In the first manuscript (Garcia et al., 2017), we described an easy-to-follow and straightforward technique to fabricate 3D printed patient-specific simulators guided by educational objectives. The anatomically correct models obtained from those methods would ultimately be better than the tubular and idealistic current training tools. Each step of the creation process was explained in Chapter 4, from the segmentation of the imaging data to the choice(s) of material(s), based on the example of an ATA model.

Designing an accurate medical simulator is complex and requires skills and techniques from multiple disciplines. It starts with the participation of medical experts to obtain optimal imaging data. Gating of the image sequence is important when organs move. It helps avoid blurry images, but also lengthens the acquisition time. When poor images are provided, time-consuming and challenging idealizations and/or improvements of the geometries are required. Moreover, not all data is easily obtainable from one imaging technique. In the case of aortic models, CT is not optimal for identifying the wall thicknesses, which has to be estimated by applying constant offsets to the lumen, but local variations would be neglected. Consequently, post imaging fixing or improving the geometries can be done but are not ideal as they may not be representative of the in vivo geometry. 
After segmentation, artifacts of the 3D parts would have to be removed and irregularities fixed by smoothing surfaces. This procedure is delicate as we do not want to deteriorate details that could be important in the representation of the area of interest. Also, too little smoothing generally increases the number of elements required to define the object while too much smoothing has the opposite effect. The control of the number of elements is important as it would affect the ease of manipulation of the object, as well as its level of details. Finally, for an accurate representation of the desired form of the model, the choices of the proper 3D printer and materials would be key. Printers do not offer the same resolution and the quality of the printing may depend on the viscosity of the liquid, when resins are used.

After an in silico model is created, the challenge is to develop a method that would give the anatomic fidelity and also physiological fidelity. It is important to investigate the possibility of creating materials with a realistic haptic rendering ideal for the practice of medical manipulations and the gain of motor skills (Armillotta et al., 2007; Kalejs \& von Segesser, 2009; Sulaiman et al., 2008; Suzuki et al., 2004). For these reasons, tunable 3D printed multi-material composites were designed to replicate properties of ATA and ATAA for educational purposes. We sought to develop fibrous structures that would have similar characteristics of the real tissue when compared with tensile testing, nano-indentation and suture retention strength of human samples. The fibers were selected to have the smallest size that the printer (Objet3 Connex500) could print without discontinuities. The impact of the embedded fibers were investigated, and we found that they could control the elasticity, strength and directional dependency of the material.

Changes of the composite structure would therefore potentially be able to replicate specific patient characteristics (tissue fidelity) varying amongst population (Chung et al., 2017; Shahmansouri et al., 2016). We chose to use a homogenous pattern of fibers, but in future studies, local variations in the patterning could be used to better represent individual pathologies. Such control of the mechanical properties would not be attained with the use of common homogeneous and isotropic materials.

We acknowledge in this work, that our composite formulation did not perfectly match the ex vivo mechanical properties of the aortic tissue. However, it is important to note that assumptions were made during this research which probably impacted the values obtained for the analyses, especially in tensile testing. Biological and non-biological samples were, without exception, considered stress-free. Therefore, the presence of residual stresses in the aortas was neglected. We used the same strain range for all analyses, even though the aortic tissue would realistically not start at a zero-stress state. Such method 
would unfortunately affect the results obtained for the material stiffness computed at a specific strain value (25\%). The latter is usually taken higher than physiological strains (5-10\% (Grøndal et al., 2012; Morrison et al., 2009)) to account the residual in vivo stresses of the tissue (Emmott et al., 2016). In addition, the absence of the residual stress in the 3D printed materials would not allow the models to recoil when cut, like vascular tissue. The compensation or replication of this mechanical response with the synthetic material has not been investigated.

Similarly, comparisons between the aortic tissues and 3D printed materials should be nuanced, as all testing methods are labor-intensive, thus only limited numbers of biological samples are evaluated in any given study. In tensile testing, number of samples ranges between 6 and 40, which leaves very little room for patient subcategorization with any statistical power (Emmott et al., 2016). Also, ascending ATAA tissue is inhomogeneous with regional variations (Choudhury et al., 2009; Della Corte et al., 2012; Iliopoulos et al., 2009) but most studies neglect this potential variability especially when a small quantity of tissue is analyzed. Additionally, several tissue samples are sometimes extracted from the same subject, thus the diversity of the patient population would remain limited (Khanafer et al., 2011; T. Matsumoto et al., 2009). Consequently, interpretations and conclusions might not be entirely representative of the patient population. Also, data would only give the general and average characteristics of the vessel, thus neglect mechanical local variations of the tissue.

In the stated objectives of the thesis, a goal was to move towards producing anatomically correct ATA and ATAA training phantoms that would replicate the deformation under pressure of real tissue (physiologic fidelity). To reach this goal, we implemented 3D printed structures presented in Garcia et al. (2018) to aortic models. Each model was then evaluated by tensile testing and SPE to investigate the dynamic accuracy of the models at different points in the cardiac cycle. Replicating this mechanical response with this new generation of models is key, as they would allow the practice of entire surgical procedures during cardiac perfusion and on pump bypass instead of limiting the practice to simple cardiac arrested individual tasks. Moreover, they could help train medical experts recognize and diagnose pathologies during surgery and from dynamic medical imaging.

The third paper helps move toward allowing surgeons to replicate a patient specific pathology prior to surgery. Using medical imaging estimates of aortic biomechanics, 3D printed patient specific models can be recreated and allow the surgeon to practice the procedure on a model with physiological fidelity. 
Previous work by our group has shown that energy loss can be used as predictors for risk assessment (Emmott et al., 2016). The results presented in my thesis shown that simulated in vivo measures co-varied with the destructive mechanical testing of the 3D printed models. In addition, the same imaging used in patients confirmed that only the material composition of our models impacted the overall physiological fidelity and the geometry of the print did not significantly change it. The dynamic echo-compatible aortic simulators were also able to reproduce motions and properties of real aortas with room-temperature water.

SPE was originally used for initial diagnoses to assess the global motion of the vessels in response to the stresses caused by blood pressure, which has only been recently used for the evaluation of the aortic properties by analyzing the local deformations of the walls in order to help surgeons to make surgical decisions (Satriano et al., 2015). It was shown that distensibility of the aortic tissue was correlating with risks of rupture. The use of echo-parameters could therefore be potentially replaced or could complete the current predictor of dangerous conditions (Di Martino et al., 2006; Wilson et al., 2003). Nowadays, surgical decisions are mainly taken in accordance of the maximum diameter of the dilatation, where 5$5.5 \mathrm{~cm}$ becomes risky for an adult (Rosamond et al., 2007; Saliba \& Sia, 2015).

The next step of this research would consist in 3D printing several models and methodically evaluate them with experienced cardiac surgeons from diverse hospitals. A collaboration with experts in medical simulation and education would be required to assess the efficiency of the training and quality of the ATA, ATAA models.

Again, the objective of this research project was to create training tools for unexperienced cardiac surgeons, where the geometries, mechanics and physiological responses were crucial. The use of 3D bio printing (or biodegradable materials) was therefore not an option, as we are currently not able to create accurate and (large) parts with this method. Moreover, it would have cost more money (need of special room conditions) than PJ 3D printers and the storage of the materials would have been more complicated.

In addition, a more detailed investigation of the fibers' geometries would be necessary to better understand their impact on the mechanics of the composites. The models presented in Paper 2 were already selected from preliminary studies achieved by a master student (Zhang et al., 2018), who played with parameters shown in Appendix 7. Same as the collagen, we assumed that the greater the undulation 
of the embedded zigzag geometries, the greater the extension at which the material stiffens. However, at this stage of the process we were limited by the technology its capacity to make thin and complex fibers.

Similarly, more investigations could be done on the bio-fluid mechanics of the models connected to a flow loop, such as the assessment of the helical flow and/ or secondary flow. Those dynamic parameters would indeed affect the mechanical responses (stresses for instance) of the walls, thus the deformations. The use of the angiography procedure, Doppler imaging or the most recent cardiac magnetic resonance (Hope et al., 2011) or 4D MRI (Harloff et al., 2010; Markl et al., 2010) methods are non-invasive and could be used to obtain velocity profiles of the models and continue the research.

\section{Practical Issues}

For the creation of models with anatomic and tissue fidelity, we had to implement the composite structure in the aortic geometry. This might look trivial, but it was a major obstacle to the project. In opposite to standardized geometries used for the physiological models, there was no specific tool that would have done it automatically and accurately on patient-specific geometries. Therefore, we were forced to develop a method requiring the use of several software to create aortic geometries, fibers and projections over the models (Appendix 5). Projection was a reliable technique but would always slightly deform the fibers as there would not be smooth surfaces after segmentation of patient aortas.

In addition, the creation of 3D printing patient aortic models was complex, especially the cleaning of the objects. Long tubular shapes with small diameters (coronaries, branches) and large curvatures are fragile when printed and thus not easy to clean with traditional tools (carving tools, waterjet). Stratasys advised us to leave them in a high PH solution for a few hours, however, we found that the resins absorbed liquids and a short analysis confirmed it by showing an increase weight of $7 \%(\mathrm{~N}=4)$ after a 3.5-hour bath (Appendix 3). Such reaction was therefore damaging the composites by changing the volumes of the inside structures (fibers and support material) and generating cracks on the outside. Consequently, we were forced to develop our own method which consisted of spraying models with a cleaning product made of ammonia (Appendix 6) to partially dissolve the support material before removing it by hand. The creation of novel 3D printing cleaning tools for specific and complex geometries could be an additional solution to improve the process. 
Finally, to avoid any variabilities in the mechanical responses of the cured printed resins, we always underwent the analyses within 48 hours after printing for the squared sample (Manuscript 2) and within a week after fabrication for the simulators with physiologic fidelity (Manuscript 3 ). According to an investigation explained in Appendix 4, samples became too brittle for testing after three weeks when they were kept in sealed bag with air. Nitrogen would help and lengthen the product lifetime up to two more weeks. The technique used to remove 3D printed parts from the platform was also important, as we did not want to break any of the fibrous structure and damage the product when it was still warm. Once the samples were collected, we protected them from light in sealed opaque bags to avoid chemical as photopolymerization. Finally, the maintenance and conditions (UV lights, print heads, cleanliness) of the machine, as well as the quality of the resins (new or almost expired) would also be crucial for consistency matters.

In conclusion, the research question of this thesis was whether 3D printed composites could be used to represent human aortic tissue needed for physical surgical simulation. The thesis has shown that we successfully developed methods to 3D print aortic models with anatomic, tissue and physiologic fidelity. We were therefore able to create medical phantoms with composite structures that would improve the current training tools and allow the practice of entire surgical procedures when being incorporated into a mock circulatory system. 


\section{Chapter 8: Original contributions to knowledge}

This research has established methods to create 3D printed aortic models with anatomical, tissue and physiological fidelities. Accurate patient-specific geometries were obtained by gated CT-Scan while the design of synthetic, 3D printed and tunable composites was guided by the composition of the aortic tissue. It was found that characteristics of the printed materials could be controlled, such as the softness, strength, and mechanical directional dependency. The simulated tissue also showed good echogenicity and matched properties of the aortic tissue.

Using 3D printing to create precise and representative aortic models could therefore represent a widely used technique to fabricate realistic aortic models for surgical simulation. The work of this thesis had important and significant advances and the novel contributions presented in this research were:

1. The development of a straightforward method to capture patient aortic geometries by CT-scan for 3D printed static rigid phantoms.

A review manuscript about the current 3D printed phantoms in medical simulation was published and based on the example of the ATA. It appeared there was lack of vascular models for teaching purposes. Each step of the method was explained and adapted for the creation of 3D printed patient-specific aortas.

2. The design of tunable fibrous composites to control material properties and replicate the biomechanics of normal and aneurysmal ascending aortas.

We based the structure of our composites on the natural composition of the aortic tissue. The study has shown that variations in the fiber geometry would affect the elasticity, strength and mechanical directional dependency of the material. Therefore, compositions and structures could be adapted in accordance to the properties that the operator wants to replicate - properties that would vary among patients and diseases.

3. The development of a method to implement the composites in the aortic models for mechanically realistic phantoms.

There is currently no specific tool that would automatically and easily implement composites in aortic geometries, especially those obtained from segmentation. We therefore had to develop a technique combining several software in order to project grids of fibers over the models. 
4. The development of a circuit to evaluate the physiological fidelity of the aortic phantoms. A mock flow circulation model was created to simulate aortas under physiological conditions for dynamic SPE. A positive displacement pump -that simulated heart cycles by changing pressureswas connected to a 3D printed aortic model while echo-parameters were evaluated.

5. The development of dynamic aortic phantoms with physiological fidelity for perfusion system. A novel generation of dynamic 3D printed aortic simulators with physiological fidelity suitable for a perfusion system was developed to practice entire cardiac surgeries.

To conclude, I made novel and meaningful contributions towards the improvement of the ascending thoracic aortic surgical simulator. The creation of such models has proven to be very complex due to the multidisciplinary nature of the project, as well as the technological constraints and limitations. Therefore, this work provides a prototype of the methods to create patient specific thoracic aortic medical simulators and identifies limitations that could be overcome with improvements in technology to evolve physical surgical simulators in general. 


\section{Chapter 9: Future work}

\subsection{Aortic model and more detailed 3D printed tissue}

The method that we developed for the implementation of the composite is time-consuming. Moreover, we should be able to change the fibrous patterns locally in order to give more realistic patient specific models. The solutions of these problem would be:

- Coding a program to implement automatically the composite/ fibrous structures in the patient specific aortic models,

- Designing more fibres and defining their properties in order to be able to replicate a larger panel of tissue characteristics,

- Mapping properties of patient-specific aortas in order to replicate local properties and 3D print more realistic models.

\subsection{Use of other 3D printed materials}

The study has shown the limitations of the current 3D printed materials. The next steps of the study would consist in:

- Creating a new generation of materials with chemical engineers,

- Trying other technologies or materials available on the market.

We only had access to the soft 3D printed material TGPF930 when we started the study. However, others have been developed by Stratasys and will be soon on the market.

\subsection{Perfusion system}

The setup of the third paper for SPE remained idealistic and could be improved for future investigations with:

- Having a $37^{\circ} \mathrm{C}$ blood-like liquid with a representative viscosity,

- More realistic colors to simulate blood. 


\subsection{Sampling}

We had a limited number of aortic samples, thus were not able to make comparisons to specific groups that could have been divided by sex, age and pathologies, for instance. To solve this limitation, we should:

- Continue evaluating ex vivo, in vivo aortic tissue for normal and pathological aortas. 


\section{Bibliography}

Adams, A. J., Wasson, E. A., Admire, J. R., Pablo Gomez, P., Babayeuski, R. A., Sako, E. Y., \& Willis, R. E. (2015). A comparison of teaching modalities and fidelity of simulation levels in teaching resuscitation scenarios. JSURG Journal of Surgical Education, 72(5), 778-785.

Armillotta, A., Bonhoeffer, P., Dubini, G., Ferragina, S., Migliavacca, F., Sala, G., \& Schievano, S. (2007). Use of rapid prototyping models in the planning of percutaneous pulmonary valved stent implantation. Proceedings of the Institution of Mechanical Engineers, Part H: Journal of Engineering in Medicine, 221(4), 407-416.

Baumgartner, D., Baumgartner, C., Mátyás, G., Steinmann, B., Löffler-Ragg, J., Schermer, E., . . . Hess, J. (2005). Diagnostic power of aortic elastic properties in young patients with Marfan syndrome. The Journal of Thoracic and Cardiovascular Surgery, 129(4), 730-739.

Biglino, G., Verschueren, P., Zegels, R., Taylor, A. M., \& Schievano, S. (2013). Rapid prototyping compliant arterial phantoms for in-vitro studies and device testing. Journal of Cardiovascular Magnetic Resonance, 15(2), 2-7.

Blanco, D., Fernandez, P., \& Noriega, A. (2014). Nonisotropic experimental characterization of the relaxation modulus for PolyJet manufactured parts. Journal of Materials Research, 29(17), 18761882.

Blue Phantom. (2013). Shop by Anatomy - Heart. Retrieved from http://www.bluephantom.com/product/Transthoracic-Echocardiography-andPericardiocentesis-Ultrasound-Training-Model.aspx?cid=411

Boltz, T. F., Pavlicek, W., Paden, R., Renno, M., Jensen, A., \& Akay, M. (2010). An anthropomorphic beating heart phantom for cardiac x-ray CT imaging evaluation. Journal of Applied Clinical Medical Physics, 11(1), 191-199. Retrieved from http://www.jacmp.org/index.php/jacmp/article/view/3129/1781

Bonow, R. O. (2008). Bicuspid aortic valves and dilated aortas: a critical review of the critical review of the ACC/AHA practice guidelines recommendations. American Journal of Cardiology, 102(1), 111-114.

Boodhwani, M., Andelfinger, G., Leipsic, J., Lindsay, T., McMurtry, M. S., Therrien, J., \& Siu, S. C. (2014). Canadian cardiovascular society position statement on the management of thoracic aortic disease. Canadian journal of cardiology, 30(6), 577-589.

Cao, P., Duhamel, Y., Olympe, G., Ramond, B., \& Langevin, F. (2013). A new production method of elastic silicone carotid phantom based on MRI acquisition using rapid prototyping technique. Paper presented at the 35th Annual International Conference of the IEEE Engineering in Medicine and Biology Society Osaka, Japan.

Carlson, R. G., Lillehei, C. W., \& Edwards, J. E. (1970). Cystic medial necrosis of the ascending aorta in relation to age and hypertension. American Journal of Cardiology, 25(4), 411-415.

Chae, M. P., Rozen, W. M., McMenamin, P. G., Findlay, M. W., Spychal, R. T., \& Hunter-Smith, D. J. (2015). Emerging applications of bedside 3D printing in plastic surgery. Frontiers in Surgery, 2, 25. doi:10.3389/fsurg.2015.00025

Chakravarthy, E., ter Haar, E., Bhat, S. S., McCoy, C. E., Denmark, T. K., \& Lotfipour, S. (2011). Simulation in medical school education: review for emergency medicine. Western Journal of Emergency Medicine, 12(4), 461-466. Retrieved from http://www.ncbi.nlm.nih.gov/pmc/articles/PMC3236168/

Chan, H. H., Siewerdsen, J. H., Vescan, A., Daly, M. J., Prisman, E., \& Irish, J. C. (2015). 3D rapid prototyping for otolaryngology-head and neck surgery: applications in image-guidance, surgical simulation and patient-specific modeling. PloS one, 10(9), e0136370.

Chen, H., Liu, Y., Slipchenko, M. N., Zhao, X., Cheng, J. X., \& Kassab, G. S. (2011). The layered structure of coronary adventitia under mechanical load. Biophysical journal, 101(11), 2555-2562. 
Choudhury, N., Bouchot, O., Rouleau, L., Tremblay, D., Cartier, R., Butany, J., . . Leask, R. L. (2009). Local mechanical and structural properties of healthy and diseased human ascending aorta tissue. Cardiovascular Pathology, 18(2), 83-91.

Chua, C. K., Chou, S. M., Lin, S. C., Eu, K. H., \& Lew, K. F. (1998). Rapid prototyping assisted surgery planning. The International Journal of Advanced Manufacturing Technology, 14(9), 624-630. doi:10.1007/BF01192281

Chung, J., Lachapelle, K., Cartier, R., Mongrain, R., \& Leask, R. L. (2017). Loss of mechanical directional dependency of the ascending aorta with severe medial degeneration. Cardiovascular Pathology, 26, 45-50. doi:http://doi.org/10.1016/i.carpath.2016.11.001

Chung, J., Lachapelle, K., Wener, E., Cartier, R., De Varennes, B., Fraser, R., \& Leask, R. L. (2014). Energy loss, a novel biomechanical parameter, correlates with aortic aneurysm size and histopathologic findings. The Journal of Thoracic and Cardiovascular Surgery, 148(3), 1082-1089.

Chuong, C. J., \& Fung, Y. C. (1984). Compressibility and constitutive equation of arterial wall in radial compression experiments. Journal of Biomechanics, 17(1), 35-40. doi:https://doi.org/10.1016/0021-9290(84)90077-0

Cloonan, A. J., Shahmirzadi, D., Li, R. X., Doyle, B. J., Konofagou, E. E., \& McGloughlin, T. M. (2014). 3Dprinted tissue-mimicking phantoms for medical imaging and computational validation applications. 3D Printing and Additive Manufacturing, 1(1), 14-23.

Clouse, W. D., Hallett Jr, J. W., Schaff, H. V., Gayari, M. M., Ilstrup, D. M., \& Melton III, L. J. (1998). Improved prognosis of thoracic aortic aneurysms: a population-based study. Jama, 280(22), 1926-1929.

Cohen, J., \& Reyes, S. A. (2015). Creation of a 3D printed temporal bone model from clinical CT data. American journal of otolaryngology, 36(5), 619-624.

Computerized Imaging Reference Systems Inc. (2014). 3 Dimensional torso phantom. Retrieved from http://www.cirsinc.com/products/new/29/3-dimensional-torso-phantom/

Conklin, L. D., \& Reardon, M. J. (2001). Technical aspects of the Ross procedure. Texas Heart Institute Journal, 28(3), 186-189. Retrieved from http://www.ncbi.nlm.nih.gov/pmc/articles/PMC101173/

Cook, D. A., Hatala, R., Brydges, R., Zendejas, B., Szostek, J. H., Wang, A. T., . . Hamstra, S. J. (2011). Technology-enhanced simulation for health professions education: a systematic review and metaanalysis. Jama, 306(9), 978-988. doi:10.1001/jama.2011.1234

De Giovanni, D., Roberts, T., \& Norman, G. (2009). Relative effectiveness of high-versus low-fidelity simulation in learning heart sounds. Medical Education, 43(7), 661-668.

de Montbrun, S. L., \& MacRae, H. (2012). Simulation in surgical education. Clinics in Colon and Rectal Surgery, 25(3), 156-165.

Deferm, S., Meyns, B., Vlasselaers, D., \& Budts, W. (2016). 3D-printing in congenital cardiology: from flatland to spaceland. Journal of Clinical Imaging Science, 6, 8. doi:10.4103/2156-7514.179408

Della Corte, A., Baldascino, F., La Marca, F., Scardone, M., Nappi, G., Cefarelli, M., . . De Feo, M. (2012). Hemostatic modifications of the Bentall procedure: imbricated proximal suture and fibrin sealant reduce postoperative morbidity and mortality rates. Texas Heart Institute Journal, 39(2), 206-210. Retrieved from http://www.ncbi.nlm.nih.gov/pmc/articles/PMC3384038/

Di Martino, E. S., Bohra, A., Vande Geest, J. P., Gupta, N., Makaroun, M. S., \& Vorp, D. A. (2006). Biomechanical properties of ruptured versus electively repaired abdominal aortic aneurysm wall tissue. Journal of vascular surgery, 43(3), 570-576.

Dickinson, K. J., Matsumoto, J., Cassivi, S. D., Reinersman, J. M., Fletcher, J. G., Morris, J. W. K. S., . M., \& Blackmon, S. H. (2015). Individualizing management of complex esophageal pathology using three-dimensional printed models. The Annals of Thoracic Surgery, 100(2), 692-697. doi:10.1016/j.athoracsur.2015.03.115

Doty, D. B., \& Doty, J. R. (2012). Cardiac surgery operative technique. Philadelphia, PA: Elsevier/Saunders. 
Driessen, N. J. B., Peters, G. W. M., Huyghe, J. M., Bouten, C. V. C., \& Baaijens, F. P. T. (2003). Remodelling of continuously distributed collagen fibres in soft connective tissues. Journal of Biomechanics, 36(8), 1151-1158. doi:https://doi.org/10.1016/S0021-9290(03)00082-4

Dutta, S., \& Krummel, T. M. (2006). Simulation: a new frontier in surgical education. Advances in surgery, 40, 249-263.

Emmott, A., Alzahrani, H., Alreishidan, M., Therrien, J., Leask, R. L., \& Lachapelle, K. (2018). Transesophageal echocardiographic strain imaging predicts aortic biomechanics: Beyond diameter. The Journal of Thoracic and Cardiovascular Surgery.

Emmott, A., Garcia, J., Chung, J., Lachapelle, K., El-Hamamsy, I., Mongrain, R., . . . Leask, R. L. (2016). Biomechanics of the ascending thoracic aorta: A clinical perspective on engineering data. Canadian journal of cardiology, 32(1), 35-47.

Evgeniou, E., \& Loizou, P. (2013). Simulation-based surgical education. ANS ANZ Journal of Surgery, 83(9), 619-623.

Finlay, H. M., McCullough, L., \& Canham, P. B. (1995). Three-dimensional collagen organization of human brain arteries at different transmural pressures. Journal of Vascular Research, 32(5), 301-312. Retrieved from http://www.karger.com/DOI/10.1159/000159104

Fox, R., Walker, J. J., \& Draycott, T. J. (2011). Medical simulation for professional development-science and practice. BJOG: An International Journal of Obstetrics \& Gynaecology, 118, 1-4.

Friedman, T., Michalski, M., Goodman, T. R., \& Brown, J. E. (2016). 3D printing from diagnostic images: a radiologist's primer with an emphasis on musculoskeletal imaging-putting the 3D printing of pathology into the hands of every physician. Skeletal Radiology, 45(3), 307-321. doi:10.1007/s00256-015-2282-6

Garcia, J., AlOmran, M., Emmott, A., Mongrain, R., Lachapelle, K., \& Leask, R. L. (2018). Tunable 3D printed multi-material composites to enhance tissue fidelity for surgical simulation. Journal of Surgical Simulation, 5, 87-98.

Garcia, J., Yang, Z., Mongrain, R., Leask, R. L., \& Lachapelle, K. (2017). 3D printing materials and their use in medical education: a review of current technology and trends for the future. BMJ Simulation and Technology Enhanced Learning. doi:10.1136/bmjstel-2017-000234

Gasser, T. C., Gallinetti, S., Xing, X., Forsell, C., Swedenborg, J., \& Roy, J. (2012). Spatial orientation of collagen fibers in the abdominal aortic aneurysm's wall and its relation to wall mechanics. Acta Biomaterialia, 8(8), 3091-3103.

Gasser, T. C., Ogden, R. W., \& Holzapfel, G. A. (2006). Hyperelastic modelling of arterial layers with distributed collagen fibre orientations. Journal of The Royal Society Interface, 3(6), 15-35.

Geyer, H., Caracciolo, G., Abe, H., Wilansky, S., Carerj, S., Gentile, F., .. . Sengupta, P. P. (2010). Assessment of myocardial mechanics using speckle tracking echocardiography: fundamentals and clinical applications. Journal of the American Society of Echocardiography, 23(4), 351-369. doi:10.1016/j.echo.2010.02.015

Gillaspie, E. A., Matsumoto, J. S., Morris, N. E., Downey, R. J., Shen, K. R., Allen, M. S., \& Blackmon, S. H. (2016). From 3-dimensional printing to 5-dimensional printing: enhancing thoracic surgical planning and resection of complex tumors. The Annals of Thoracic Surgery, 101(5), 1958-1962. doi:http://dx.doi.org/10.1016/j.athoracsur.2015.12.075

Giovinco, N. A., Dunn, S. P., Dowling, L., Smith, C., Trowell, L., Ruch, J. A., \& Armstrong, D. G. (2012). A novel combination of printed 3-dimensional anatomic templates and computer-assisted surgical simulation for virtual preoperative planning in charcot foot reconstruction. YJFAS The Journal of Foot and Ankle Surgery, 51(3), 387-393.

Government of Canada. (2016). Cardiovascular disease. Public Health Agency of Canada. Retrieved from http://cbpp-pcpe.phac-aspc.gc.ca/fr/chronic-diseases/cardiovascular-diseases/

Greenwald, S. (2007). Ageing of the conduit arteries. The Journal of pathology, 211(2), 157-172. 
Grøndal, N., Bramsen, M. B., Thomsen, M. D., Rasmussen, C. B., \& Lindholt, J. S. (2012). The cardiac cycle is a major contributor to variability in size measurements of abdominal aortic aneurysms by ultrasound. European Journal of Vascular and Endovascular Surgery, 43(1), 30-33.

Guo, N., \& Leu, M. C. (2013). Additive manufacturing: technology, applications and research needs. Frontiers of Mechanical Engineering, 8(3), 215-243.

Hager, A., Kaemmerer, H., Rapp-Bernhardt, U., Blücher, S., Rapp, K., Bernhardt, T. M., . . Hess, J. (2002). Diameters of the thoracic aorta throughout life as measured with helical computed tomography. The Journal of Thoracic and Cardiovascular Surgery, 123(6), 1060-1066.

Hamstra, S. J., Brydges, R., Hatala, R., Zendejas, B., \& Cook, D. A. (2014). Reconsidering fidelity in simulation-based training. Academic medicine : journal of the Association of American Medical Colleges, 89(3), 387-392.

Harloff, A., Nußbaumer, A., Bauer, S., Stalder, A. F., Frydrychowicz, A., Weiller, C., . . Markl, M. (2010). In vivo assessment of wall shear stress in the atherosclerotic aorta using flow-sensitive 4D MRI. Magnetic Resonance in Medicine: An Official Journal of the International Society for Magnetic Resonance in Medicine, 63(6), 1529-1536.

Hiratzka, L. F., Bakris, G. L., Beckman, J. A., Bersin, R. M., Carr, V. F., Casey, D. E., . . Williams, D. M. (2010). Guidelines for the diagnosis and management of patients with thoracic aortic disease: executive summary. Journal of the American College of Cardiology, 55(14), 1509-1544.

Hirst, A. E., Johns, V. J., \& Kime, S. W. (1958). Dissecting aneurysm of the aorta: a review of 505 cases. Medicine, 37(3), 217.

Ho, D., \& Sun, Z. (2017). Modelling of aortic aneurysm and aortic dissection through 3D printing. Journal of Medical Radiation Sciences, 64(1), 10-17.

Hochman, J. B., Kraut, J., Kazmerik, K., \& Unger, B. J. (2014). Generation of a 3D printed temporal bone model with internal fidelity and validation of the mechanical construct. Otolaryngology-Head and Neck Surgery, 150(3), 448-454.

Hochman, J. B., Rhodes, C., Wong, D., Kraut, J., Pisa, J., \& Unger, B. (2015). Comparison of cadaveric and isomorphic three-dimensional printed models in temporal bone education. LARY The Laryngoscope, 125(10), 2353-2357.

Hoit, B. D. (2011). Strain and strain rate echocardiography and coronary artery disease. Circulation: Cardiovascular Imaging, 4(2), 179-190.

Holzapfel, G. A., Sommer, G., \& Regitnig, P. (2004). Anisotropic mechanical properties of tissue components in human atherosclerotic plaques. Transactions of the ASME-K-Journal of Biomechanical Engineering, 126(5), 657-665.

Hope, M. D., Hope, T. A., Crook, S. E., Ordovas, K. G., Urbania, T. H., Alley, M. T., \& Higgins, C. B. (2011). 4D flow CMR in assessment of valve-related ascending aortic disease. JACC: Cardiovascular Imaging, 4(7), 781-787.

Humphrey, J. D. (2010). Cardiovascular solid mechanics : cells, tissues, and organs. In. Retrieved from http://dx.doi.org/10.1007/978-0-387-21576-1

Huotilainen, E., Jaanimets, R., Valášek, J., Marcián, P., Salmi, M., Tuomi, J., . . Wolff, J. (2014). Inaccuracies in additive manufactured medical skull models caused by the DICOM to STL conversion process. Journal of Cranio-Maxillofacial Surgery, 42(5), e 259-265.

Iliopoulos, D. C., Kritharis, E. P., Giagini, A. T., Papadodima, S. A., \& Sokolis, D. P. (2009). Ascending thoracic aortic aneurysms are associated with compositional remodeling and vessel stiffening but not weakening in age-matched subjects. The Journal of Thoracic and Cardiovascular Surgery, 137(1), 101-109.

Isselbacher, E. M. (2005). Thoracic and abdominal aortic aneurysms. Circulation, 111(6), 816-828.

Kalejs, M., \& von Segesser, L. K. (2009). Rapid prototyping of compliant human aortic roots for assessment of valved stents. Interactive cardiovascular and thoracic surgery, 8(2), 182-186. 
Kee, S., Larsen, E., Paluch, K., Sinke, R., Yan, K. C., Pilla, J. J., \& Chun, X. (2010). Development of a dynamic heart phantom prototype for magnetic resonance imaging. Paper presented at the Bioengineering Conference, Proceedings of the 2010 IEEE 36th Annual Northeast, New York.

Khanafer, K., Duprey, A., Zainal, M., Schlicht, M., Williams, D. M., \& Berguer, R. (2011). Determination of the elastic modulus of ascending thoracic aortic aneurysm at different ranges of pressure using uniaxial tensile testing. The Journal of Thoracic and Cardiovascular Surgery, 142(3), 682-686.

Kim, G. D., \& Oh, Y. T. (2008). A benchmark study on rapid prototyping processes and machines: quantitative comparisons of mechanical properties, accuracy, roughness, speed, and material cost. Proceedings of the Institution of Mechanical Engineers, Part B: Journal of Engineering Manufacture, 222(2), 201-215.

Kim, M. S., Hansgen, A. R., \& Carroll, J. D. (2008). Use of rapid prototyping in the care of patients with structural heart disease. Trends in cardiovascular medicine, 18(6), 210-216.

Kneebone, R., \& Baillie, S. (2008). Contextualized simulation and procedural skills: a view from medical education. Journal of Veterinary Medical Education, 35(4), 595-598.

Koken Co. (2005). Physical assessment trainer. Retrieved from http://www.kokenmpc.co.jp/english/products/life simulation models/medical education/Im084/index.html

Kolyva, C., Biglino, G., Pepper, J. R., \& Khir, A. W. (2012). A mock circulatory system with physiological distribution of terminal resistance and compliance: application for testing the intra-aortic balloon pump. Artificial Organs, 36(3), E62-E70. doi:10.1111/j.1525-1594.2010.01071.x

Kondo, K., Harada, N., Masuda, H., Sugo, N., Terazono, S., Okonogi, S., . . . Nemoto, M. (2016). A neurosurgical simulation of skull base tumors using a 3D printed rapid prototyping model containing mesh structures. Acta Neurochir Acta Neurochirurgica : The European Journal of Neurosurgery, 158(6), 1213-1219.

Kong, X., Nie, L., Zhang, H., Wang, Z., Ye, Q., Tang, L., . . . Li, J. (2016). Do 3D printing models improve anatomical teaching about hepatic segments to medical students? A randomized controlled study. World Journal of Surgery, 40(8), 1969-1976. doi:10.1007/s00268-016-3541-y

Kouchoukos, N. T., \& Dougenis, D. (1997). Surgery of the thoracic aorta. The New England journal of medicine, 336(26), 1876-1888.

Kurenov, S. N., Ionita, C., Sammons, D., \& Demmy, T. L. (2015). Three-dimensional printing to facilitate anatomic study, device development, simulation, and planning in thoracic surgery. The Journal of Thoracic and Cardiovascular Surgery, 149(4), 973-979.

Kuzmik, G. A., Sang, A. X., \& Elefteriades, J. A. (2012). Natural history of thoracic aortic aneurysms. Journal of vascular surgery, 56(2), 565-571.

Kyoto Kagaku Co. (1999-2012). Cardiology patient simulator "K". Retrieved from https://www.kyotokagaku.com/products/detail01/m84-s.html

Laerdal Medical. (2014). Harvey ${ }^{\circledast}$ The Cardiopulmonary Patient Simulator. Retrieved from http://www.laerdal.com/ca/harvey

Länne, T., Sonesson, B., Bergqvist, D., Bengtsson, H., \& Gustafsson, D. (1992). Diameter and compliance in the male human abdominal aorta: influence of age and aortic aneurysm. European journal of vascular surgery, 6(2), 178-184.

Lasheras, J. C. (2007). The biomechanics of arterial aneurysms. Annual Review of Fluid Mechanics, 39, 293319.

Lee, K. H. K., Grantham, H., \& Boyd, R. (2008). Comparison of high-and low-fidelity mannequins for clinical performance assessment. Emergency Medicine Australasia, 20(6), 508-514.

Levi, D., Rampa, F., Barbieri, C., Pricca, P., Franzini, A., \& Pezzotta, S. (2002). True 3D reconstruction for planning of surgery on malformed skulls. Child's Nervous System, 18(12), 705-706. 
Li, A. E., Kamel, I., Rando, F., Anderson, M., Kumbasar, B., Lima, J. A., \& Bluemke, D. A. (2004). Using MRI to assess aortic wall thickness in the multiethnic study of atherosclerosis: distribution by race, sex, and age. American Journal of Roentgenology, 182(3), 593-597.

Li, A. E., Kamel, I., Rando, F., Anderson, M., Kumbasar, B., Lima, J. A. C., \& Bluemke, D. A. (2004). Using $\mathrm{MRI}$ to assess aortic wall thickness in the multiethnic study of atherosclerosis: distribution by race, sex, and age. American Journal of Roentgenology, 182(3), 593-597.

Lujan, H. L., \& DiCarlo, S. E. (2006). First-year medical students prefer multiple learning styles. Advances in physiology education, 30(1), 13-16.

Malik, H. H., Darwood, A. R. J., Shaunak, S., Kulatilake, P., Abdulrahman, A., Mulki, O., \& Baskaradas, A. (2015). Three-dimensional printing in surgery: a review of current surgical applications. Journal of Surgical Research, 199(2), 512-522.

Mann, B. D. (2008). Surgery A Competency-Based Companion E-Book: With STUDENT CONSULT Online Access: Elsevier Health Sciences.

Mann, B. D. (2008). Surgery: A competency-based companion Philadelphia, PA: Elsevier Health Sciences.

Maragiannis, D., Jackson, M. S., Igo, S. R., Schutt, R. C., Connell, P., Grande-Allen, J., . . Little, S. H. (2015). Replicating patient-specific severe aortic valve stenosis with functional 3D modeling. Circulation Cardiovascular imaging, 8(10).

Maran, N. J., \& Glavin, R. J. (2003). Low-to high-fidelity simulation-a continuum of medical education? Medical Education, 37(s1), 22-28.

Markl, M., Schumacher, R., Küffer, J., Bley, T. A., \& Hennig, J. (2005). Rapid vessel prototyping: vascular modeling using 3t magnetic resonance angiography and rapid prototyping technology. Magnetic Resonance Materials in Physics, Biology and Medicine, 18(6), 288-292. doi:10.1007/s10334-0050019-6

Markl, M., Wallis, W., Brendecke, S., Simon, J., Frydrychowicz, A., \& Harloff, A. (2010). Estimation of global aortic pulse wave velocity by flow-sensitive 4D MRI. Magnetic Resonance in Medicine, 63(6), 15751582.

Marr, M., Hemmert, K., Nguyen, A. H., Combs, R., Annamalai, A., Miller, G., . . Cohen, S. M. (2012). Team play in surgical education: a simulation-based study. Journal of Surgical Education, 69(1), 63-69.

Martin, C., Pham, T., \& Sun, W. (2011). Significant differences in the material properties between aged human and porcine aortic tissues. European Journal of Cardio-Thoracic Surgery, 40(1), 28-34. doi:10.1016/j.ejcts.2010.08.056

Matsumoto, E. D., Hamstra, S. J., Radomski, S. B., \& Cusimano, M. D. (2002). The effect of bench model fidelity on endourological skills: a randomized controlled study. The Journal of urology, 167(3), 1243-1247.

Matsumoto, J. S., Morris, J. M., Foley, T. A., Williamson, E. E., Leng, S., McGee, K. P., .. Vrtiska, T. J. (2015). Three-dimensional physical modeling: Applications and experience at mayo clinic. Radiographics, 35(7), 1989-2006.

Matsumoto, T., Fukui, T., Tanaka, T., Ikuta, N., Ohashi, T., Kumagai, K., ... Sato, M. (2009). Biaxial tensile properties of thoracic aortic aneurysm tissues. Journal of Biomechanical Science and Engineering, 4(4), 518-529.

Matsumoto, T., Goto, T., Furukawa, T., \& Sato, M. (2004). Residual stress and strain in the lamellar unit of the porcine aorta: experiment and analysis. Journal of Biomechanics, 37(6), 807-815.

McLeod, A. J., Moore, J., Guiraudon, G. M., Jones, D. L., Campbell, G., \& Peters, M. T. (2011). Surgical phantom for off-pump mitral valve replacement. Paper presented at the SPIE, San Diego.

Meurling, L., Hedman, L., Lidefelt, K.-J., Escher, C., Felländer-Tsai, L., \& Wallin, C.-J. (2014). Comparison of high- and low equipment fidelity during paediatric simulation team training: a case control study. BMC Medical Education, 14(1), 221. doi:10.1186/1472-6920-14-221

Miller, D. W. (1977). The practice of coronary artery bypass surgery. New York: Plenum Medical. 
Moore, W. S., \& Jimenez, J. C. (2011). A handbook of vascular disease management. Retrieved from http://www.123library.org/book details/?id=52476

Morrison, T. M., Choi, G., Zarins, C. K., \& Taylor, C. A. (2009). Circumferential and longitudinal cyclic strain of the human thoracic aorta: age-related changes. Journal of vascular surgery, 49(4), 1029-1036. doi:10.1016/j.jvs.2008.11.056

Morton, L. F., \& Barnes, M. J. (1982). Collagen polymorphism in the normal and diseased blood vessel wall: investigation of collagens types I, III and V. Atherosclerosis, 42(1), 41-51.

Mowry, S. E., Jammal, H., Myer, C., Solares, C. A., \& Weinberger, P. (2015). A novel temporal bone simulation model using 3D printing techniques. Otology \& neurotology : official publication of the American Otological Society, American Neurotology Society [and] European Academy of Otology and Neurotology, 36(9), 1562-1565.

Mulvany, M. J., \& Aalkjaer, C. (1990). Structure and function of small arteries. Physiological reviews, 70(4), 921-961.

Munshi, F., Lababidi, H., \& Alyousef, S. (2015). Low- versus high-fidelity simulations in teaching and assessing clinical skills. Journal of Taibah University Medical Sciences, 10(1), 12-15. doi:https://doi.org/10.1016/j.jtumed.2015.01.008

Negi, S., Dhiman, S., \& Kumar Sharma, R. (2014). Basics and applications of rapid prototyping medical models. Rapid Prototyping Journal, 20(3), 256-267.

Noecker, A. M., Chen, J. F., Zhou, Q., White, R. D., Kopcak, M. W., Arruda, M. J., \& Duncan, B. W. (2006). Development of patient-specific three-dimensional pediatric cardiac models. American Society for Artificial Internal Organs : 1992, 52(3), 349-353.

Norman, G., Dore, K., \& Grierson, L. (2012). The minimal relationship between simulation fidelity and transfer of learning. Medical Education, 46(7), 636-647.

O'Rourke, M. F., Staessen, J. A., Vlachopoulos, C., \& Duprez, D. (2002). Clinical applications of arterial stiffness; definitions and reference values. American Journal of Hypertension, 15(5), 426-444.

Ogden, K. M., Aslan, C., Ordway, N., Diallo, D., Tillapaugh-Fay, G., \& Soman, P. (2015). Factors affecting dimensional accuracy of 3-D printed anatomical structures derived from CT data. Journal of Digital Imaging, 28(6), 654-663.

Okamoto, R. J., Wagenseil, J. E., DeLong, W. R., Peterson, S. J., Kouchoukos, N. T., \& Sundt III, T. M. (2002). Mechanical properties of dilated human ascending aorta. Annals of biomedical engineering, 30(5), 624-635.

Okamoto, R. J., Xu, H., Kouchoukos, N. T., Moon, M. R., \& Sundt, T. M. (2003). The influence of mechanical properties on wall stress and distensibility of the dilated ascending aorta. The Journal of Thoracic and Cardiovascular Surgery, 126(3), 842-850.

Oliver, W. C., \& Pharr, G. M. (1992). An improved technique for determining hardness and elastic modulus using load and displacement sensing indentation experiments. Journal of Materials Research, 7(06), 1564-1583.

Oxman, N., Tsai, E., \& Firstenberg, M. (2012). Digital anisotropy: a variable elasticity rapid prototyping platform. Virtual and Physical Prototyping, 7(4), 261-274. doi:10.1080/17452759.2012.731369

Paige, J. B., \& Morin, K. H. (2013). Simulation fidelity and cueing: A systematic review of the literature. Clinical Simulation in Nursing, 9(11), e481-e489.

Pazos, V., Mongrain, R., \& Tardif, J. C. (2009). Polyvinyl alcohol cryogel: optimizing the parameters of cryogenic treatment using hyperelastic models. Journal of the Mechanical Behavior of Biomedical Materials, 2(5), 542-549. doi:http://dx.doi.org/10.1016/j.jmbbm.2009.01.003

Pazos, V., Mongrain, R., \& Tardif, J. C. (2010). Deformable mock stenotic artery with a lipid pool. Journal of Biomechanical Engineering, 132(3), 0345011-0345014.

Pepley, D. F., Sonntag, C. C., Prabhu, R. S., Yovanoff, M. A., Han, D. C., Miller, S. R., \& Moore, J. Z. (2018). Building ultrasound phantoms with modified polyvinyl chloride: a comparison of needle insertion 
forces and sonographic appearance with commercial and traditional simulation materials. Simulation in healthcare: journal of the Society for Simulation in Healthcare.

Perloff, J. K., Child, J. S., \& Aboulhosn, J. (2009). Congenital Heart Disease in Adults. Retrieved from http://www.clinicalkey.com/dura/browse/bookChapter/3-s2.0-C20090498106

Petrini, J., Eriksson, M. J., Caidahl, K., \& Larsson, M. (2018). Circumferential strain by velocity vector imaging and speckle-tracking echocardiography: validation against sonomicrometry in an aortic phantom. Clinical physiology and functional imaging, 38(2), 269-277.

Pham, T., Martin, C., Elefteriades, J. A., \& Sun, W. (2013). Biomechanical characterization of ascending aortic aneurysm with concomitant bicuspid aortic valve and bovine aortic arch. Acta Biomaterialia, 9(8), 7927-7936.

Pisano, C., Maresi, E., Balistreri, C. R., Candore, G., Merlo, D., Fattouch, K., . . Ruvolo, G. (2011). Histological and genetic studies in patients with bicuspid aortic valve and ascending aorta complications. Interactive cardiovascular and thoracic surgery, 14(3), 300-306.

Ploch, C. C., Mansi, C. S. S. A., Jayamohan, J., \& Kuhl, E. (2016). Using 3D printing to create personalized brain models for neurosurgical training and preoperative planning. World neurosurgery, 90, 668674. doi:http://dx.doi.org/10.1016/j.wneu.2016.02.081

Presotto, L., Bettinardi, V., Petta, P., \& Gilardi, M. C. (2012). A compact dynamic phantom to assess the effect of motion in cardiac PET and SPECT studies. Paper presented at the Nuclear Science Symposium and Medical Imaging Conference (NSS/MIC), 2012 IEEE.

Qayumi, K., Pachev, G., Zheng, B., Ziv, A., Koval, V., Badiei, S., \& Cheng, A. (2014). Status of simulation in health care education: an international survey. advances in medical education and practice, 2014(default), 457-467.

Ramphal, P. S., Coore, D. N., Craven, M. P., Forbes, N. F., Newman, S. M., Coye, A. A., . . Silvera, B. C. (2005). A high fidelity tissue-based cardiac surgical simulator. European Journal of Cardio-Thoracic Surgery, 27(5), 910-916. doi:10.1016/j.ejcts.2004.12.049

Rampoldi, V., Trimarchi, S., Eagle, K. A., Nienaber, C. A., Oh, J. K., Bossone, E., . . Cooper, J. V. (2007). Simple risk models to predict surgical mortality in acute type $A$ aortic dissection: the International Registry of Acute Aortic Dissection score. The Annals of Thoracic Surgery, 83(1), 55-61.

Reiter, M., \& Major, Z. (2011). A combined experimental and simulation approach for modelling the mechanical behaviour of heterogeneous materials using rapid prototyped microcells. Virtual and Physical Prototyping, 6(2), 111-120. doi:10.1080/17452759.2011.586949

Rengier, F., Mehndiratta, A., Tengg-Kobligk, H., Zechmann, C. M., Unterhinninghofen, R., Kauczor, H.-U., \& Giesel, F. L. (2010). 3D printing based on imaging data: review of medical applications. International Journal of Computer Assisted Radiology and Surgery, 5(4), 335-341. doi:10.1007/s11548-010-0476-x

Ripley, B., Kelil, T., Cheezum, M. K., Goncalves, A., Di Carli, M. F., Rybicki, F. J., . . Blankstein, R. (2016). 3D printing based on cardiac CT assists anatomic visualization prior to transcatheter aortic valve replacement. Journal of Cardiovascular Computed Tomography, 10(1), 28-36. doi:http://dx.doi.org/10.1016/i.jcct.2015.12.004

Rooke, T. W., Sullivan, T. M., \& Jaff, M. I. R. (2007). Vascular medicine and endovascular interventions. [Malden, MA]: Blackwell Futura.

Rosamond, W., Flegal, K., Friday, G., Furie, K., \& Go, A. (2007). Heart disease and stroke statistics-2007 update: a report from the American Heart Association Statistics Committee and Stroke Statistics Subcommittee. Circulation, 5(115), 69-171.

Rosen, K. R. (2008). The history of medical simulation. Journal of Critical Care, 23(2), 157-166. doi:http://dx.doi.org/10.1016/j.jcrc.2007.12.004

Saliba, E., \& Sia, Y. (2015). The ascending aortic aneurysm: when to intervene? IJC Heart \& Vasculature, 6, 91-100. doi:https://doi.org/10.1016/j.ijcha.2015.01.009 
Salloum, C., Lim, C. G. T., Fuentes, L., Osseis, M., Luciani, A., \& Azoulay, D. (2016). Fusion of information from 3D printing and surgical robot: an innovative minimally technique illustrated by the resection of a large celiac trunk aneurysm. World Journal of Surgery, 40(1), 245-247.

Satriano, A., Rivolo, S., Martufi, G., Finol, E. A., \& Di Martino, E. S. (2015). In vivo strain assessment of the abdominal aortic aneurysm. Journal of Biomechanics, 48(2), 354-360. doi:10.1016/j.jbiomech.2014.11.016

Schlatmann, T. J., \& Becker, A. E. (1977). Histologic changes in the normal aging aorta: implications for dissecting aortic aneurysm. The American Journal of Cardiology, 39(1), 13-20.

Schmauss, D., Gerber, N., \& Sodian, R. (2013). Three-dimensional printing of models for surgical planning in patients with primary cardiac tumors. The Journal of Thoracic and Cardiovascular Surgery, 145(5), 1407-1408.

Schmauss, D., Haeberle, S., Hagl, C., \& Sodian, R. (2015). Three-dimensional printing in cardiac surgery and interventional cardiology: a single-centre experience. European Journal of Cardio-Thoracic Surgery, 47(6), 1044-1052.

Schmauss, D., Schmitz, C., Bigdeli, A. K., Weber, S., Gerber, N., Beiras-Fernandez, A., . . Sodian, R. (2012). Three-dimensional printing of models for preoperative planning and simulation of transcatheter valve replacement. The Annals of Thoracic Surgery, 93(2), e31-33.

Sellke, F. W., \& Ruel, M. (2010). Atlas of cardiac surgical techniques a volume in the Surgical techniques atlas series. Philadelphia, PA: Saunders/Elsevier.

Shah, K., Ahmed, J., Shenoy, N., \& Srikant, N. (2017). How different are students and their learning styles? International Journal of Research in Medical Sciences, 1(3), 212-215.

Shahmansouri, N., Alreshidan, M., Emmott, A., Lachapelle, K., El-Hamamsy, I., Cartier, R., . . Mongrain, R. (2016). Investigation on the regional loss factor and its anisotropy for aortic aneurysms. Materials, 9(11), 867. Retrieved from http://www.mdpi.com/1996-1944/9/11/867

Shiraishi, I., Yamagishi, M., Hamaoka, K., Fukuzawa, M., \& Yagihara, T. (2010). Simulative operation on congenital heart disease using rubber-like urethane stereolithographic biomodels based on 3D datasets of multislice computed tomography. European Journal of Cardio-Thoracic Surgery, 37(2), 302-306. doi:10.1016/j.ejcts.2009.07.046

Singhal, A. J., Shetty, V., Bhagavan, K. R., Ragothaman, A., Shetty, V., Koneru, G., \& Agarwala, M. (2016). Improved surgery planning using 3-D printing: a case study. Indian Journal of Surgery, 78(2), 100104. doi:10.1007/s12262-015-1326-4

Sipila, H. T., Teras, M., Kokki, T., \& Knuuti, J. (2007). A moving heart phantom for dual gated cardiac PET/CT studies. Paper presented at the Nuclear Science Symposium Conference Record, 2007.

Soares, P. V., de Almeida Milito, G., Pereira, F. A., Reis, B. R., Soares, C. J., de Sousa Menezes, M., \& de Freitas Santos-Filho, P. C. (2013). Rapid prototyping and 3D-virtual models for operative dentistry education in Brazil. Journal of dental education, 77(3), 358-363.

Spottiswoode, B. S., Van den Heever, D. J., Chang, Y., Engelhardt, S., Du Plessis, S., Nicolls, F., ... Gretschel, A. (2013). Preoperative three-dimensional model creation of magnetic resonance brain images as a tool to assist neurosurgical planning. Stereotactic and functional neurosurgery, 91(3), 162-169.

Sulaiman, A., Boussel, L., Taconnet, F., Serfaty, J.-M., Alsaid, H., Attia, C., . . Douek, P. (2008). In vitro nonrigid life-size model of aortic arch aneurysm for endovascular prosthesis assessment. European Journal of Cardio-Thoracic Surgery, 33(1), 53-57. doi:10.1016/j.ejcts.2007.10.016

Suzuki, M., Ogawa, Y., Kawano, A., Hagiwara, A., Yamaguchi, H., \& Ono, H. (2004). Rapid prototyping of temporal bone for surgical training and medical education. Acta Oto-Laryngologica, 124(4), 400402.

Taber, L. A., \& Humphrey, J. D. (2001). Stress-modulated growth, residual stress and vascular heterogeneity. Journal of Biomechanical Engineering, 123(6), 528-535. doi:10.1115/1.1412451 
Tam, M. D. B. S., Laycock, S. D., Brown, J. R. I., \& Jakeways, M. (2013). 3D printing of an aortic aneurysm to facilitate decision making and device selection for endovascular aneurysm repair in complex neck anatomy. Journal of Endovascular Therapy, 20(6), 863-867.

Tang, P. C. Y., Coady, M. A., Lovoulos, C., Dardik, A., Aslan, M., Elefteriades, J. A., \& Tellides, G. (2005). Hyperplastic cellular remodeling of the media in ascending thoracic aortic aneurysms. Circulation, 112(8), 1098-1105.

Tavakoli, V., Negahdar, M. J., Kendrick, M., Alshaher, M., Stoddard, M., \& Amini, A. A. (2012). A biventricular multimodal (MRI/Ultrasound) cardiac phantom. Paper presented at the 34th Annual International Conference of the IEEE EMBS, San Diego.

Teixeira, R., Moreira, N., Baptista, R., Barbosa, A., Martins, R., Castro, G., \& Providência, L. (2012). Circumferential ascending aortic strain and aortic stenosis. European Heart JournalCardiovascular Imaging, 14(7), 631-641.

The Chamberlain Group. (2014a). Heart. Retrieved from http://www.thecgroup.com/products/heart

The Chamberlain Group. (2014b). Products. Retrieved from http://www.thecgroup.com/products

Trehan, K., Kemp, C. D., \& Yang, S. C. (2014). Simulation in cardiothoracic surgical training: Where do we stand? The Journal of Thoracic and Cardiovascular Surgery, 147(1), 18-24.e12. doi:10.1016/j.jtcvs.2013.09.007

Tremblay, D., \& Leask, R. L. (2011). Remodelling and pathology development associated with aneurysmal ascending aortic tissues. The Canadian Journal of Chemical Engineering, 89(1), 13-22.

Tremblay, D., Zigras, T., Cartier, R., Leduc, L., Butany, J., Mongrain, R., \& Leask, R. L. (2009). A comparison of mechanical properties of materials used in aortic arch reconstruction. The Annals of Thoracic Surgery, 88(5), 1484-1491.

Tsamis, A., Krawiec, J. T., \& Vorp, D. A. (2013). Elastin and collagen fibre microstructure of the human aorta in ageing and disease: a review. Journal of The Royal Society Interface, 10(83), 20121004.

Turkbey, E. B., Jain, A., Johnson, C., Redheuil, A., Arai, A. E., Gomes, A. S., . . Eng, J. (2014). Determinants and normal values of ascending aortic diameter by age, gender, and race/ethnicity in the multiethnic study of atherosclerosis Journal of Magnetic Resonance Imaging, 39(2), 360-368.

Ventola, C. L. (2014). Medical applications for 3D printing: current and projected uses. Pharmacy and Therapeutics, 39(10), 704.

Vitarelli, A., Giordano, M., Germanò, G., Pergolini, M., Cicconetti, P., Tomei, F., . . Bruno, P. (2010). Assessment of ascending aorta wall stiffness in hypertensive patients by tissue Doppler imaging and strain Doppler echocardiography. Heart (British Cardiac Society), 96(18), 1469-1474.

Von Ahn, L., Maurer, B., McMillen, C., Abraham, D., \& Blum, M. (2008). Human-based character recognition via web security measures. Science, 321(5895), 1465-1468.

Vorp, D. A., Schiro, B. J., Ehrlich, M. P., Juvonen, T. S., Ergin, M. A., \& Griffith, B. P. (2003). Effect of aneurysm on the tensile strength and biomechanical behavior of the ascending thoracic aorta. The Annals of Thoracic Surgery, 75(4), 1210-1214.

Vukicevic, M., Puperi, D. S., Grande-Allen, K. J., \& Little, S. H. (2016). 3D printed modeling of the mitral valve for catheter-based structural interventions. Annals of biomedical engineering, 1-12.

Walenga, R. L., Longest, P. W., \& Sundaresan, G. (2014). Creation of an in vitro biomechanical model of the trachea using rapid prototyping. Journal of Biomechanics, 47(8), 1861-1868.

Wang, A., \& Bashore, T. M. (2009). Valvular heart disease. Dordrecht; New York: Humana Press.

Wang, K., Wu, C., Qian, Z., Zhang, C., Wang, B., \& Vannan, M. A. (2016). Dual-material 3D printed metamaterials with tunable mechanical properties for patient-specific tissue-mimicking phantoms. Additive Manufacturing, 12, Part A, 31-37. doi:http://dx.doi.org/10.1016/j.addma.2016.06.006 
Wang, K., Zhao, Y., Chang, Y.-H., Qian, Z., Zhang, C., Wang, B., . . . Wang, M.-J. (2016). Controlling the mechanical behavior of dual-material 3D printed meta-materials for patient-specific tissuemimicking phantoms. Materials \& Design, 90, 704-712.

Wang, M., \& Lakatta, E. G. (2002). Altered regulation of matrix metalloproteinase- 2 in aortic remodeling during aging. Hypertension, 39(4), 865-873.

Waran, V., Narayanan, V., Karuppiah, R., Owen, S. L., \& Aziz, T. (2014). Utility of multimaterial 3D printers in creating models with pathological entities to enhance the training experience of neurosurgeons. Journal of Neurosurgery, 120(2), 489-492.

Williams, J. B., Peterson, E. D., Zhao, Y., O'Brien, S. M., Andersen, N. D., Miller, D. C., ... Hughes, G. C. (2012). Contemporary results for proximal aortic replacement in North America. Journal of the American College of Cardiology, 60(13), 1156-1162.

Wilson, K. A., Lee, A. J., Hoskins, P. R., Fowkes, F. G., Ruckley, C. V., \& Bradbury, A. W. (2003). The relationship between aortic wall distensibility and rupture of infrarenal abdominal aortic aneurysm. Journal of vascular surgery, 37(1), 112-117.

Wolinsky, H., \& Glagov, S. (1964). Structural basis for the static mechanical properties of the aortic media. Circulation research, 14(5), 400-413.

Wong, K. V., \& Hernandez, A. (2012). A review of additive manufacturing. ISRN Mechanical Engineering, 2012, 1-10.

Wurm, G., Lehner, M., Tomancok, B., Kleiser, R., \& Nussbaumer, K. (2011). Cerebrovascular biomodeling for aneurysm surgery: simulation-based training by means of rapid prototyping technologies. Surgical innovation, 18(3), 294-306.

Yan, K. C., McDonough, M. K., Pilla, J. J., \& Xu, C. (2011). Stiffness characterization using a dynamic heart phantom and magnetic resonance imaging. Paper presented at the International Mechanical Engineering Congress \& Exposition Denver, Colorado.

Yang, Z. (2016). Development of 3D printed patient specific ascending aortic training models for cardiac surgery. (Master degree). McGill, Retrieved from http://digitool.Library.McGill.CA/R/?func=dbinjump-full\&object id=141345 Available from http://worldcat.org /z-wcorg/ database.

Zhang, J., Zhong, Y., \& Gu, C. (2018). Deformable models for surgical simulation: a survey. IEEE reviews in biomedical engineering, 11, 143-164. doi:10.1109/RBME.2017.2773521 
Appendix

\section{Equi-biaxial tensile testing}

Stress and strain definitions

Arteries have non-linear visco-elastic behavior as the tissue exhibits viscous and elastic characteristics under deformations with a time-dependent strain rate. Many definitions exist for the stress and the strain but we used the engineering stress $\mathrm{S}$ and strain e suitable for small deformations.

$S=\frac{F}{A_{o}}$

$e=\frac{L-L_{0}}{L_{0}}$

$F$ is the recorded force, when $L_{0}$ and $L$ are the initial length and the length after deformation. $A_{0}$ stands for the initial cross-section area calculated with the measured thickness and lengths of the specimen. The engineering stress does not consider the decrease of the cross-section area during the loading cycles. Once the stresses and strains have been calculated from the experimentation, a constitutive model can then fit the data.

\section{Non-linear continuum mechanics}

We applied the theory of continuum mechanics thus assumed infinitely divisible and locally homogeneous matters. Moreover, we simplified the conditions and assumed the materials (aortic tissue, 3D printed composites) to be isotropic, hyperelastic and incompressible. The incompressibility is often used to characterize the arterial walls as a consequence of a large amount of intracellular and extracellular water in the tissue (70-80\% by weight) preventing the material from changing its volume when being pressurized (Chuong \& Fung, 1984; Humphrey, 2010).

For each deformation, displacements are expressed with vectors $u(x)$ to describe the motion of each point in the solid where $\mathrm{x}$ and $\mathrm{X}$ are the positions in the deformed and reference configurations respectively (Figure 47):

$X=x+u(x)$

Displacement gradient tensor:

$\nabla u=\frac{\partial u}{\partial x}$

Deformation gradient tensor with the identity tensor I:

$F=\nabla x=\frac{\partial x}{\partial x}=I+\nabla u$ 
The Jacobian describes the change of volume produced by deformations and is expressed with stretches in the principal directions $\lambda_{1,2,3} . J=1$ because of the incompressibility.

$J=\frac{d_{V}}{d_{V_{0}}}=\operatorname{det}(F)=\lambda_{1} \lambda_{2} \lambda_{3}$

The right Cauchy-Green deformation tensor $C$, also called a material tensor, describes how the length and angles change between the positions:

$C=F^{T} F$

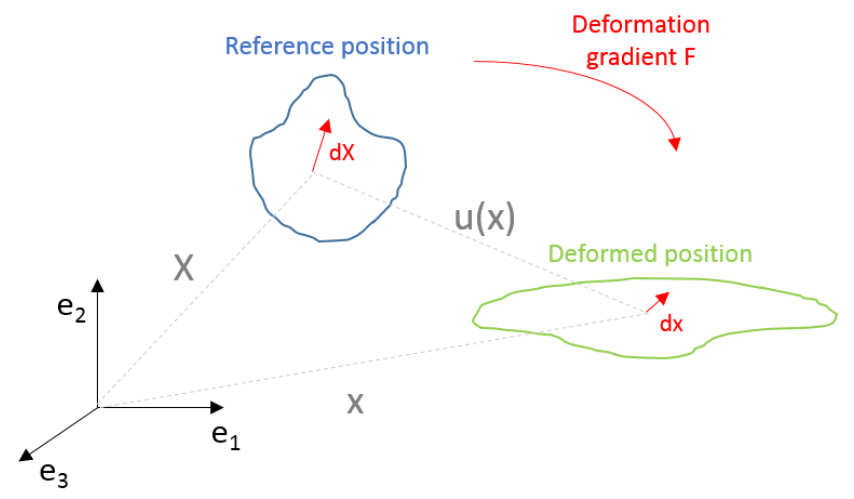

Figure 47. Deformation gradient $\mathrm{F}$ from the reference (blue) to the deformed position (green).

\section{Hyperelastic model}

For hyperelastic and isotropic materials, we chose to represent the elastic of the aortic tissue by the strainenergy function Mooney-Rivlin where the energy stored is independent from the path.

$\Psi=\sum_{\substack{1+j=1 \\ i, j=0, \ldots, n}}^{n} c_{i j}\left(\overline{I_{1}}-1\right)^{i}\left(\overline{I_{2}}-1\right)^{j}+\frac{\kappa}{2}(J-1)^{2}$

$\mathrm{K}$ is the bulk modulus, $\mathrm{C}_{\mathrm{ij}}$ are the material parameters and $\mathrm{I}_{1,2}$ the invariants.

In equi-biaxial tension: $\lambda_{1}=\lambda, \lambda_{2}=\lambda$ and $\lambda_{3}=\lambda^{-2}$ and the invariants are:

$\left\{\begin{array}{c}I_{1}=\operatorname{trace}(C)=\lambda_{1}{ }^{2}+\lambda_{2}{ }^{2}+\lambda_{3}{ }^{2}=2 \lambda^{2}+\lambda^{-4} \\ I_{2}=\frac{\operatorname{trace}\left(C^{2}\right)-\operatorname{trace}(C)^{2}}{2}=\lambda_{1}{ }^{2} \lambda_{2}{ }^{2}+\lambda_{2}{ }^{2} \lambda_{3}{ }^{2}+\lambda_{3}{ }^{2} \lambda_{1}{ }^{2}=2 \lambda^{-2}+\lambda^{4}\end{array}\right.$

Therefore, after simplifications the second order $(n=2)$ Mooney-Rivlin will be:

$$
\begin{aligned}
\Psi & =c_{10}\left(I_{1}-3\right)+c_{01}\left(I_{2}-3\right) \\
& =c_{10}\left(2 \lambda^{2}+\lambda^{-4}-3\right)+c_{01}\left(2 \lambda^{-2}+\lambda^{4}-3\right)
\end{aligned}
$$

The differences of Cauchy stresses in the principal directions $\sigma_{1,2,3}$ for an incompressible hyperelastic are:

$\sigma_{1}-\sigma_{3}=\sigma_{2}-\sigma_{3}=\lambda_{1} \frac{\partial \Psi}{\partial \lambda_{1}}-\lambda_{3} \frac{\partial \Psi}{\partial \lambda_{3}}=\lambda_{2} \frac{\partial \Psi}{\partial \lambda_{2}}-\lambda_{3} \frac{\partial \Psi}{\partial \lambda_{3}}$ 
$\sigma_{3}=0$ in equi-biaxial tension and Cauchy stresses can be calculated with the following relation and converted in engineering stress:

$\sigma_{1}=\sigma_{2}=2 c_{10}\left(\lambda^{2}-\lambda^{-4}\right)-2 c_{01}\left(\lambda^{-2}-\lambda^{4}\right)$

$\mathrm{S}_{1}=\mathrm{S}_{2}=2 \mathrm{c}_{10}\left(\lambda-\lambda^{-5}\right)+2 \mathrm{c}_{01}\left(\lambda^{3}-\lambda^{-3}\right)$ 


\section{Compositions of the resins}

\section{Thermal gravimetric analysis}

The thermal gravimetric analysis (or TGA) is a method measuring sample weights over time as a function of increasing temperature. It gives information on the decomposition reactions of materials (phase changes, absorption, desorption) by investigating the thermal stability of their systems.

The method used with air was:

1: Ramp $20.00^{\circ} \mathrm{C} / \mathrm{min}$ to $550.00^{\circ} \mathrm{C}$,

2: Select Air,

3: Ramp $20.00^{\circ} \mathrm{c} / \mathrm{min}$ to $700.00^{\circ} \mathrm{c}$.

The Weight-Temperature curves of Verowhite and Tangoplus FullCure 930 presented three significant weight losses $\left(200^{\circ} \mathrm{C}, 460^{\circ} \mathrm{C}\right.$ and $500^{\circ} \mathrm{C}$ ) while the support material only had two $\left(225^{\circ} \mathrm{C}\right.$ and $500^{\circ} \mathrm{C}$ ) (Figure 38). In Figure 38 (d), we can see that Verowhite would be less affected at a given temperature when compared to Tangoplus FullCure 930 and the support material.

Those changes occurred at very high temperatures, thus we wouldn't affect the material for normal use of the simulators. Moreover, Verowhite and Tangoplus FullCure 930 had decomposition at similar temperatures thus were made of the same chemical compounds. Finally, Verowhite would be more resistant than Tangoplus FullCure 930 and the support material. 
a)

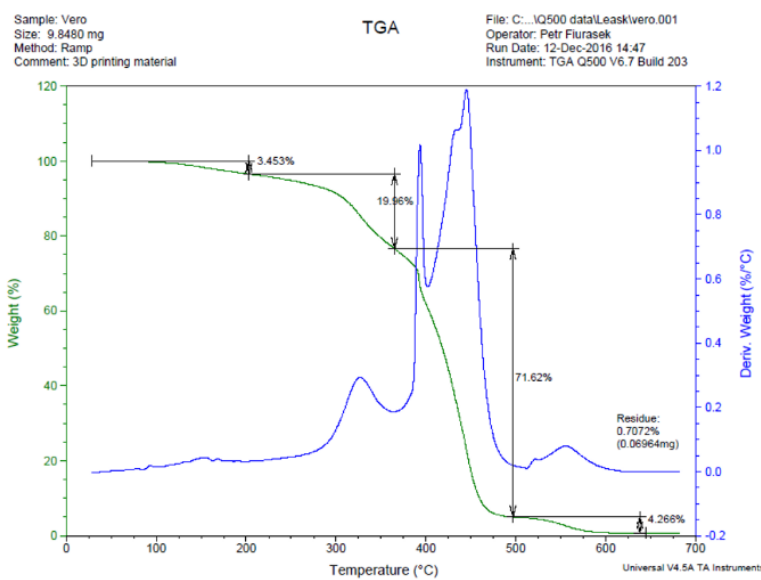

c)

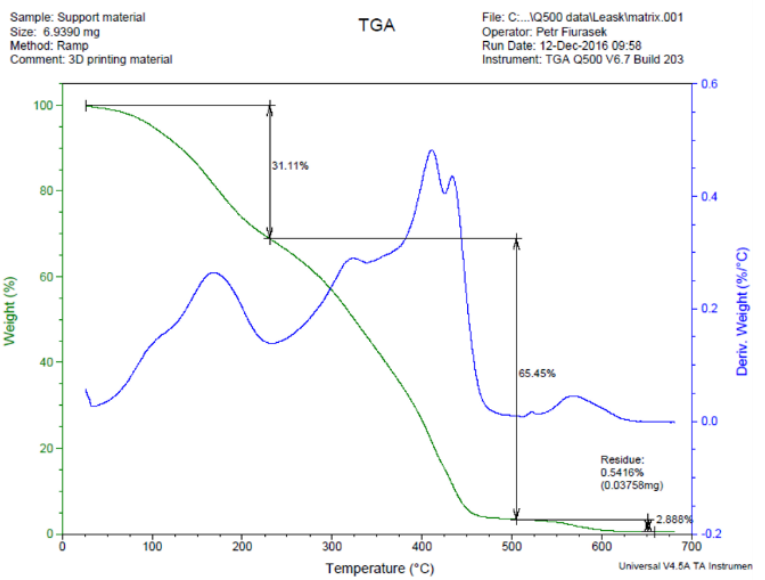

b)
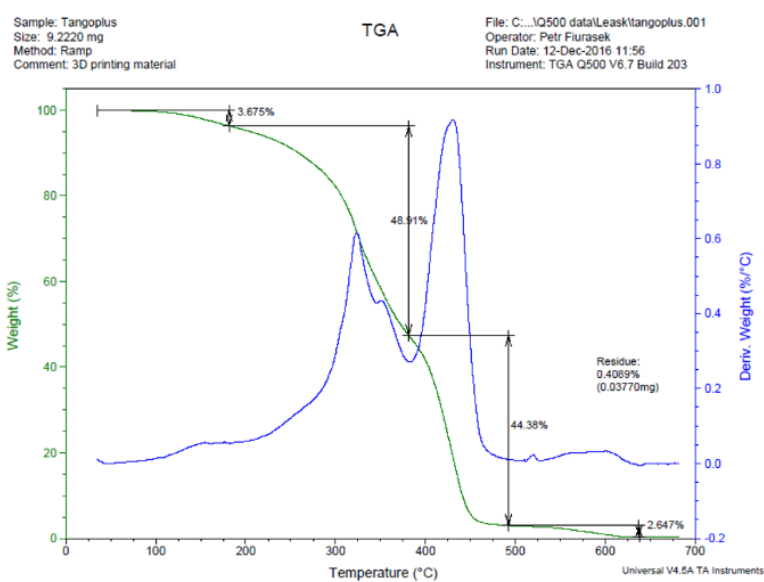

d)

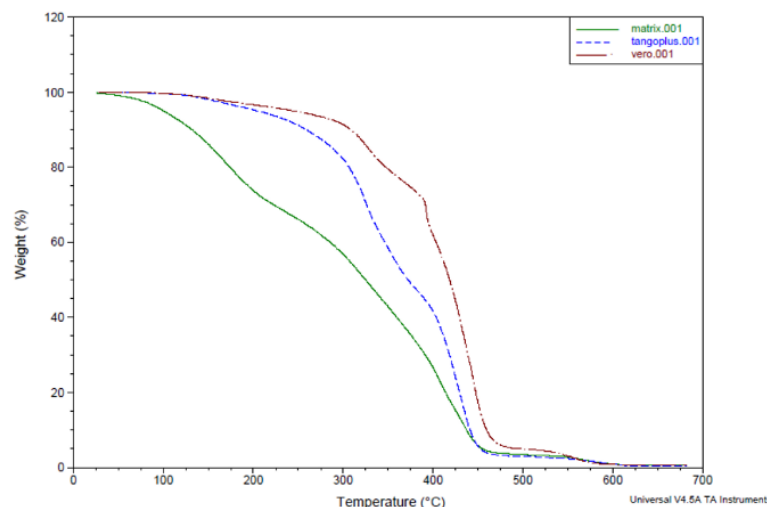

Figure 48. Weight-Temperature (green) and Derivative weight-Temperature (blue) curves for TGA analyses of a) Verowhite, b) Tangoplus FullCure 930, the c) Support material, as well as a graphic showing all Weight-Temperature curves in d).

\section{Differential scanning calorimetry}

The differential scanning calorimetry (DSC) is a method to investigate the thermal characteristics of substances like polymers by analyzing the amount of heat required to increase the temperature of the sample.

Tzero Aluminium pans were used for the experiments which consisted on:

1: Equilibrate at $-60.00^{\circ} \mathrm{C}$,

2: Ramp $10.00{ }^{\circ} \mathrm{C} / \mathrm{min}$ to $100.00^{\circ} \mathrm{C}$,

3: Mark end of cycle 0,

4: Ramp $10.00^{\circ} \mathrm{c} / \mathrm{min}$ to $-60.00^{\circ} \mathrm{C}$,

5: Mark end of cycle 0 , 
6: Ramp $10.00^{\circ} \mathrm{C} / \mathrm{min}$ to $100.00^{\circ} \mathrm{C}$,

7: Mark end of cycle 0.

Graphics in Figure 49 showed Heat flow-Temperature curves composed of the first heat cycle (in blue), cool cycle (in red) and second heat cycle (in green). Verowhite and Tangoplus FullCure 930 presented changes in heat capacity at approximately $50^{\circ} \mathrm{C}$ and $-20^{\circ} \mathrm{C}$ respectively (midpoint temperatures) for glass transitions. By contrast, the support material did not present specific reaction while we increased the temperature.

DSC showed a beginning of glass transition at $25^{\circ} \mathrm{C}$ for the Verowhite, which corresponds to regular room temperatures. Using $3 \mathrm{D}$ printed resins at $37^{\circ} \mathrm{C}$ to replicate physiological conditions could therefore start a material reaction and damage the samples.

a)

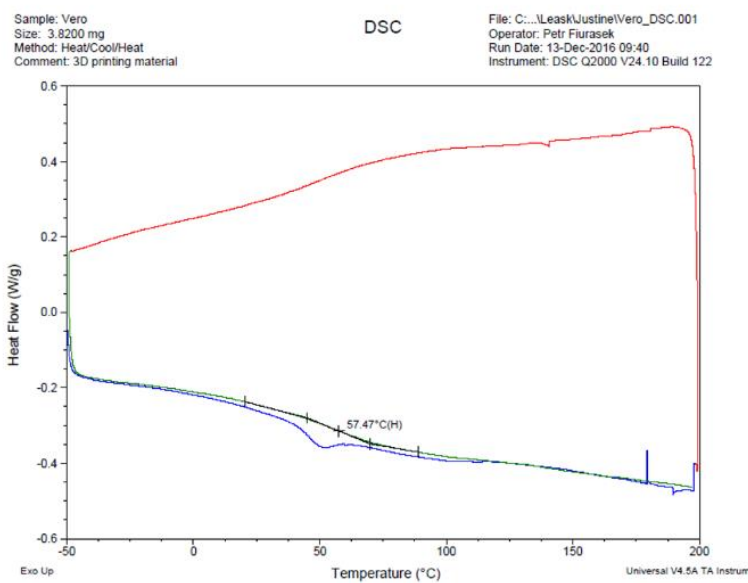

c)

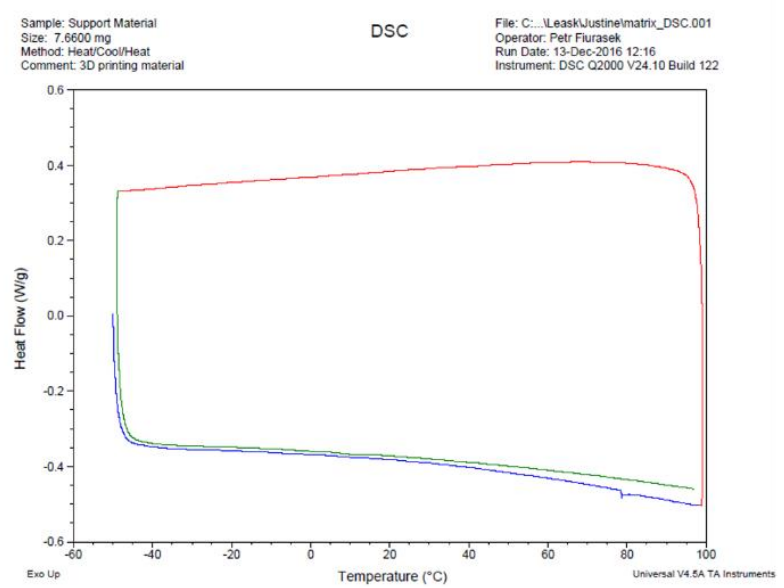

b)

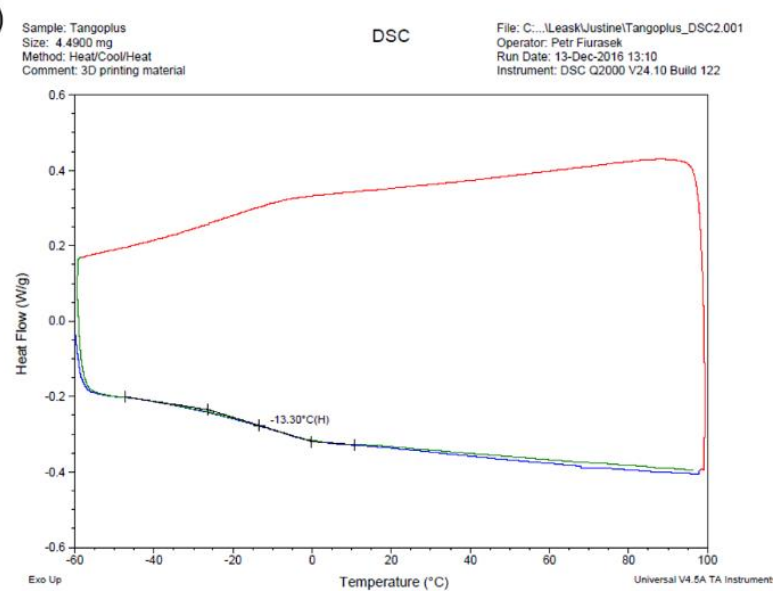

Figure 49. Heat flow-Temperature curves for DSC analyses of a) Verowhite, b) Tangoplus FullCure 930 and c) Support material. The first heat cycle is in blue, the cool cycle is in red and the second heat cycle in green. 


\section{Hydrophilic resins}

An investigation was carried out to determine if the 3D printed materials were absorbing water after being in a bath for 3.5 hours.

We found that the weight of the three-material composites $(N=4)$ showed a $7 \pm 2 \%$ increase caused by the absorption of water (Table 6) when samples $(\mathrm{N}=4)$ did not vary after the same amount of time in a dry environment (Table 7). A p =0.0009 was found for an Unpaired t-test.

Therefore, the differences between the two studies confirmed the hydrophilic property of the resins.

Table 6. Weights of the samples before and after a 3.5 hours water bath.

\begin{tabular}{lll}
\hline Weight before bath $\mathbf{( g )}$ & $\begin{array}{l}\text { Weight after } \mathbf{3 . 5} \text { hours bath } \\
\text { (g) }\end{array}$ & Difference \\
\hline 0.0310 & 0.0335 & $7 \%$ \\
\hline 0.0304 & 0.0324 & $6 \%$ \\
\hline 0.0328 & 0.0344 & $5 \%$ \\
\hline 0.1417 & 0.1567 & $10 \%$ \\
\hline & Average & $\mathbf{7 \%}$ \\
\cline { 2 - 3 } & SD & $\mathbf{2 \%}$ \\
\hline
\end{tabular}

Table 7. Weights of the samples before and after 3.5 hours.

\begin{tabular}{lll}
\hline $\mathbf{T}=\mathbf{0} \mathbf{s}(\mathbf{g})$ & After $\mathbf{3 . 5}$ hours $\mathbf{( g )}$ & Difference \\
\hline 0.0304 & 0.0305 & $0 \%$ \\
\hline 0.0300 & 0.0301 & $0 \%$ \\
\hline 0.0304 & 0.0306 & $1 \%$ \\
\hline 0.0315 & 0.0314 & $0 \%$ \\
\hline & Average & $\mathbf{0 \%}$ \\
\cline { 2 - 3 } & SD & $\mathbf{0 \%}$ \\
& &
\end{tabular}




\section{3D printed composites over time}

A study was carried out to investigate the evolution of the material properties over time. We kept samples from light in sealed bags filled with air, as well as in containers with nitrogen. Two parameters were calculated from tensile testing, such as the apparent elastic modulus at $25 \%$ strains and the energy loss. We had $\mathrm{N}=3$ samples per week.

The composites kept in sealed bags with air became very brittle thus not testable after 3 weeks. This can be confirmed by the large and abnormal standard deviation obtained for the third week (Figure 50). The use of nitrogen helped in the storage of the 3D printed resins as we were able to test them up to 5 weeks. Nevertheless, we have not found large variations of the properties over time.

To conclude, material properties did not vary over time, but the resins became brittle. The use of nitrogen lengthened the product lifetime up to 2 weeks.
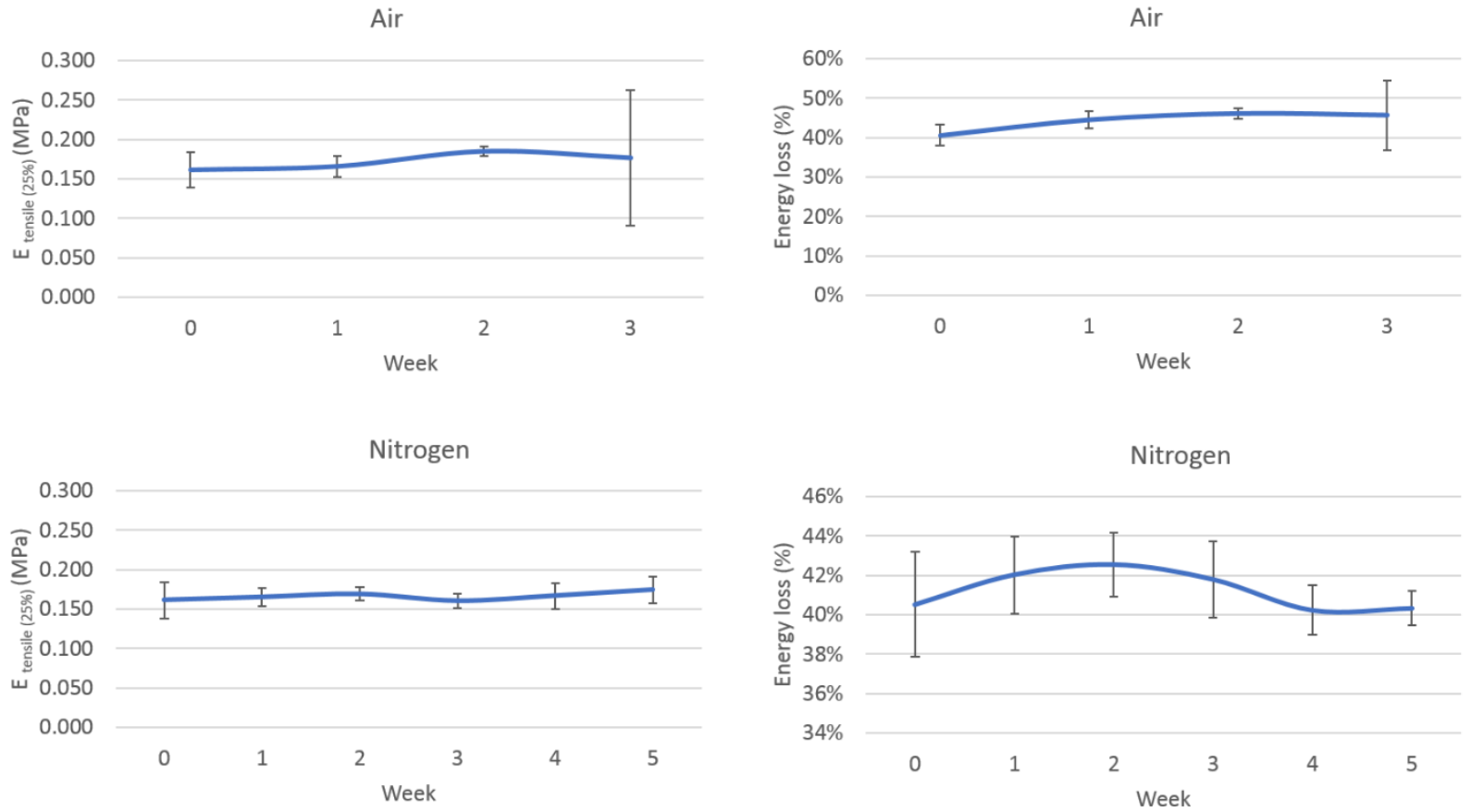

Figure 50. Variations over time of the apparent elastic modulus ( $E_{\text {tensile (25\%) }}(\mathrm{MPa})$ ) and energy loss (\%) for the three-material composite kept in air and nitrogen. 


\section{Implementation of the composite structure in the aortic geometries for static mechanically realistic phantoms}

One of the objectives of the thesis was to create static mechanically realistic phantoms (Objective 2.2) which were aortic models made of composite structures developed in Paper 2 (Garcia et al., 2018). The implementation of the synthetic material to the aortic geometries could be performed as follows.

\section{Creation of the inner structure}

First, we created two distinct aortic geometries from the lumen by adding two different offsets with Rhinoceros 3D (Nobert McNeel \& Associates, Seattle, WA, USA). Each of the aorta was used to lay down one layer of fibers.

In the case of a patient-specific geometry that would automatically be saved in STL format after the segmentation, we needed to create intermediate surfaces in order to be able to do the projection. For this reason, we used a reverse engineering tool named RhinoResurf to create surfaces over the multiple triangles constituting the STL geometry (Trunhoo, Network Technology, Nanjing, Jiangsu, China) (see Figure 51).

In parallel, grids of fibers were made by computer-aided design (Solidworks, Dassault Systems, Waltham, MA, USA) and were projected with Rhinoceros 3D over each aortic model (one per offset) for the two layers - as required for the composites. At the end, we simply had to save the two layers of fibers alone in one first STL file (inner structure).

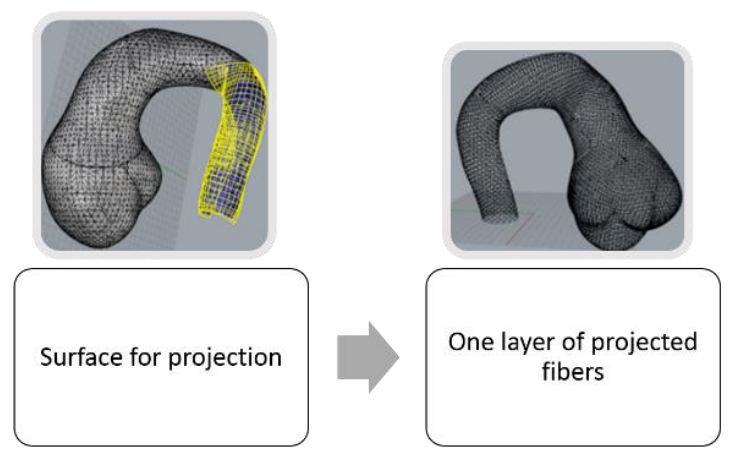

Figure 51. Creation of an intermediate surface over the STL geometry for the projection of one layer of fibers.

\section{Creation of the outer structure}

To implement the two layers of fibers, we needed an outer structure. This is why, we created a $2 \mathrm{~mm}$ offset aortic model obtained from the lumen after segmentation. We assumed a $2 \mathrm{~mm}$ thickness aortic 
wall (Arthur E Li et al., 2004). Boolean operations were then used to remove the fibers from the geometry with Netfabb (Autodesk, California, USA). A new STL format file could be finally saved (outer structure), if we limit our simulator to a two-material composite.

For the three-material composites, there was a last step which consisted in creating a hollow by controlling the shell thickness of the structure. We took the previous model and "dug" into it for the creation of a new void that was then used for the insertion of the support material during the printing. It resulted in a new STL file (outer structure) without fibers and with a hollow made for the support material.

\section{Assembly of the STL files for the printing}

As explained by Garcia et al. (2017), one STL file corresponds to only one specific 3D printing material and with the method previously described, we created two of them, such as

- $\quad$ one file for the two layers of fibers = inner structure,

- $\quad$ one file for the $2 \mathrm{~mm}$-thickness aortic structure without fibers (with or without extra hollow) = outer structure.

The fibers were made of rigid Vero when the outside was in soft TGPF930. 


\section{Ammonia}

The formula of Ammonia is $\mathrm{NH}_{3}$ (Figure 52). It is a compound of nitrogen and hydrogen that is miscible with water and gives basic aqueous solution.

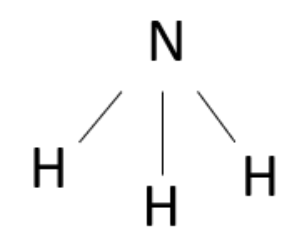

Figure 52. Ammonia formula 


\section{Tunable parameters of the fibers in the composites}

The rigid fibers used in Paper 2 and 3 were previously investigated in a Master thesis (Zhang et al., 2018). We changed several parameters including the diameter of the filets, the amplitude of the zigzags and the length (also known as a period for the sine function) as shown in Figure 53.

a)

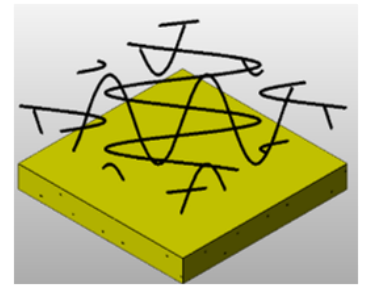

b)

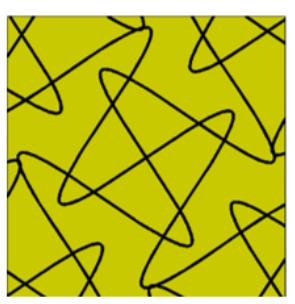

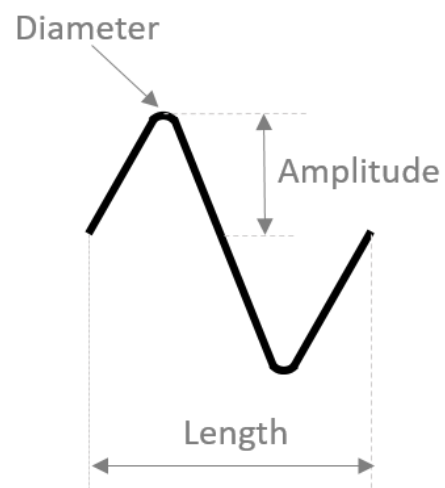

Figure 53. a) Fibers embedded in the composite and the b) Tunable parameters (Diameter, Amplitude and Length) of the fibers. 